\title{
THE TWO-DIMENSIONAL KPZ EQUATION IN THE ENTIRE SUBCRITICAL REGIME
}

\author{
FRANCESCO CARAVENNA, RONGFENG SUN, AND NIKOS ZYGOURAS
}

\begin{abstract}
We consider the KPZ equation in space dimension 2 driven by space-time white noise. We showed in previous work that if the noise is mollified in space on scale $\varepsilon$ and its strength is scaled as $\hat{\beta} / \sqrt{|\log \varepsilon|}$, then a transition occurs with explicit critical point $\hat{\beta}_{c}=\sqrt{2 \pi}$. Recently Chatterjee and Dunlap showed that the solution admits subsequential scaling limits as $\varepsilon \downarrow 0$, for sufficiently small $\hat{\beta}$. We prove here that the limit exists in the entire subcritical regime $\hat{\beta} \in\left(0, \hat{\beta}_{c}\right)$ and we identify it as the solution of an additive Stochastic Heat Equation, establishing so-called Edwards-Wilkinson fluctuations. The same result holds for the directed polymer model in random environment in space dimension 2 .
\end{abstract}

\section{INTRODUCTION AND MAIN RESULTS}

We present first our results for the two-dimensional KPZ equation, and then similar results for its discrete analogue, the directed polymer model in random environment in dimension $2+1$. We close the introduction with an outline of the rest of the paper.

1.1. KPZ IN Two Dimensions. The KPZ equation is a stochastic PDE, formally written as

$$
\partial_{t} h(t, x)=\frac{1}{2} \Delta h(t, x)+\frac{1}{2}|\nabla h(t, x)|^{2}+\beta \xi(t, x), \quad t \geqslant 0, x \in \mathbb{R}^{d},
$$

where $\xi(t, x)$ is the space-time white noise, and $\beta>0$ governs the strength of the noise. It was introduced by Kardar, Parisi and Zhang [KPZ86] as a model for random interface growth, and has since been an extremely active area of research for both physicists and mathematicians. The equation is ill-posed due to the singular term $|\nabla h|^{2}$ which is undefined, because $\nabla h$ is expected to be a distribution (generalized function).

In spatial dimension $d=1$, these difficulties can be bypassed by considering the socalled Cole-Hopf solution $h:=\log u$, where $u$ is defined as the solution of the multiplicative Stochastic Heat Equation $\partial_{t} u=\frac{1}{2} \Delta u+\beta \xi u$, which is linear and well-posed in dimension $d=1$, by classical Ito theory. On large space-time scales, the Cole-Hopf solution exhibits the same fluctuations as many exactly solvable one-dimensional interface growth models, all belonging to the so-called KPZ universality class. See the surveys [C12, QS15 for reviews on the extensive literature. Few results are known in higher dimensions (see below).

Along a different line, intense research has been carried out in recent years to make sense of the solutions of the KPZ equation and other singular stochastic PDEs. A robust theory was lacking until the seminal work by Hairer [H13] and his subsequent theory of regularity structures [H14]. Since then, a few alternative approaches have been developed, including

Date: July 3, 2019.

2010 Mathematics Subject Classification. Primary: 60H15; Secondary: 35R60, 82B44, 82D60.

Key words and phrases. KPZ Equation, Stochastic Heat Equation, White Noise, Directed Polymer Model, Edwards-Wilkinson Fluctuations, Continuum Limit, Renormalization. 
the theory of paracontrolled distributions by Gubinelli, Imkeller, and Perkowski [GIP15], the theory of energy solutions by Gonçalves and Jara [GJ14, and the renormalization approach by Kupiainen [K16]. All these approaches are only applicable to KPZ in space dimension $d=1$, where the equation is so-called subcritical, in the sense that the non-linearity vanishes in the small scale limit with a scaling that preserves the linear and the noise terms in the equation. In the language of renormalization groups, the KPZ equation in $d=1$ is superrenormalizable (see e.g. [K16]), while regarded as a disordered system, it would be called disorder relevant (see e.g. [H74], G10], and [CSZ17a, CSZ17b]).

In this paper we focus on $d=2$, which for KPZ is the critical dimension (the renormalizable or disorder marginal case). To define a solution to (1.1), we follow the standard approach and consider a spatially mollified version $\xi^{\varepsilon}:=j_{\varepsilon} * \xi$ of the noise, where $\varepsilon>0$, $j \in C_{c}\left(\mathbb{R}^{2}\right)$ is a probability density on $\mathbb{R}^{2}$ with $j(x)=j(-x)$, and $j_{\varepsilon}(x):=\varepsilon^{-2} j(x / \varepsilon)$. The key question is whether it is possible to replace $\beta \xi$ in (1.1) by $\beta_{\varepsilon} \xi^{\varepsilon}-C_{\varepsilon}$, for suitable constants $\beta_{\varepsilon}, C_{\varepsilon}$, such that the corresponding solution $h^{\varepsilon}$ converges to a non-trivial limit as $\varepsilon \downarrow 0$.

It turns out that in space dimension $d=2$ the right way to tune the noise strength is

$$
\beta_{\varepsilon}:=\hat{\beta} \sqrt{\frac{2 \pi}{\log \varepsilon^{-1}}}, \quad \text { for some } \hat{\beta} \in(0, \infty),
$$

and to consider the following mollified $K P Z$ equation (with $\|j\|_{2}^{2}:=\int_{\mathbb{R}^{2}} j(x)^{2} \mathrm{~d} x$ ):

$$
\partial_{t} h^{\varepsilon}=\frac{1}{2} \Delta h^{\varepsilon}+\frac{1}{2}\left|\nabla h^{\varepsilon}\right|^{2}+\beta_{\varepsilon} \xi^{\varepsilon}-C_{\varepsilon}, \quad \text { where } C_{\varepsilon}:=\beta_{\varepsilon}^{2} \varepsilon^{-2}\|j\|_{2}^{2} .
$$

For simplicity, we take $h^{\varepsilon}(0, \cdot) \equiv 0$ as initial datum. If we define

$$
u^{\varepsilon}(t, x):=e^{h^{\varepsilon}(t, x)},
$$

then, by Ito's formula, $u^{\varepsilon}$ solves the mollified multiplicative Stochastic Heat Equation (SHE):

$$
\partial_{t} u^{\varepsilon}=\frac{1}{2} \Delta u^{\varepsilon}+\beta_{\varepsilon} u^{\varepsilon} \xi^{\varepsilon}, \quad u^{\varepsilon}(0, \cdot) \equiv 1 .
$$

In CSZ17b] we investigated the finite-dimensional distributions of the mollified KPZ solution $h^{\varepsilon}$ as $\varepsilon \downarrow 0$. In particular, we discovered in [CSZ17b, Section 2.3] that there is a transition in the one-point distribution as $\hat{\beta}$ varies, with critical value $\hat{\beta}_{c}:=1$ : For any $t>0$,

$$
h^{\varepsilon}(t, x) \underset{\varepsilon \downarrow 0}{\stackrel{d}{\longrightarrow}}\left\{\begin{array}{ll}
\sigma_{\hat{\beta}} Z-\frac{1}{2} \sigma_{\hat{\beta}}^{2} & \text { if } \hat{\beta}<1 \\
-\infty & \text { if } \hat{\beta} \geqslant 1
\end{array} \quad \text { with } \quad \sigma_{\hat{\beta}}^{2}:=\log \frac{1}{1-\hat{\beta}^{2}}, \quad Z \sim N(0,1) .\right.
$$

(Note that the limiting distribution does not depend on $t>0$.) This can be viewed as a weak disorder to stronger disorder transition, where we borrow terminology from the directed polymer model (see Section [1.2). It was also shown in [CSZ17b] that in the subcritical regime $\hat{\beta}<\hat{\beta}_{c}:=1$ the $k$-point distribution of $h^{\varepsilon}$ asymptotically factorizes: for any finite set of distinct points $\left(x_{i}\right)_{1 \leqslant i \leqslant k}$, the random variables $\left(h^{\varepsilon}\left(t, x_{i}\right)\right)_{1 \leqslant i \leqslant k}$ converge as $\varepsilon \downarrow 0$ to independent Gaussians.

It is natural to investigate the fluctuations of $h^{\varepsilon}$, regarded as a random field, as $\varepsilon \downarrow 0$. This is what Chatterjee and Dunlap recently addressed in [CD18]. They actually considered a variant of the mollified KPZ equation (1.3), where $\beta_{\varepsilon}$ is placed in front of the non-linearity instead of the noise, namely,

$$
\partial_{t} \widetilde{h}^{\varepsilon}=\frac{1}{2} \Delta \widetilde{h}^{\varepsilon}+\frac{1}{2} \beta_{\varepsilon}\left|\nabla \tilde{h}^{\varepsilon}\right|^{2}+\xi^{\varepsilon} .
$$


However, there is a simple relation between $\tilde{h}^{\varepsilon}$ in (1.7) and $h^{\varepsilon}$ in (1.3) (see Appendix A):

$$
\tilde{h}^{\varepsilon}(t, x)-\mathbb{E}\left[\tilde{h}^{\varepsilon}(t, x)\right]=\frac{1}{\beta_{\varepsilon}}\left(h^{\varepsilon}(t, x)-\mathbb{E}\left[h^{\varepsilon}(t, x)\right]\right),
$$

therefore working with $\widetilde{h}^{\varepsilon}$ or $h^{\varepsilon}$ is equivalent.

The main result in [CD18] is that for any fixed $t>0$, when $\hat{\beta}$ is sufficiently small, the centered solution $\widetilde{h}^{\varepsilon}(t, \cdot)-\mathbb{E}\left[\widetilde{h}^{\varepsilon}(t, \cdot)\right]$, viewed as a random distributions on $\mathbb{R}^{2}$, admits nontrivial weak subsequential limits as $\varepsilon \downarrow 0$ (in a negative Hölder space). As a matter of fact, CD18 considered the KPZ equation (1.7) on the two-dimensional torus $\mathbf{T}^{2}$, for technical reasons, but it is reasonable to believe that their results should also hold on $\mathbb{R}^{2}$.

The perturbative approach followed by Chatterjee and Dunlap [CD18] is limited to $\hat{\beta}$ sufficiently small, and it does not prove the existence of a unique limiting random field. Our main result shows that such a limit indeed exists, in the entire subcritical regime $\hat{\beta} \in(0,1)$, and identifies it as the solution of an additive SHE with a non-trivial noise strength (that depends explicitly on $\hat{\beta}$ ). This is commonly called Edwards-Wilkinson fluctuations [EW82].

Theorem 1.1 (Edwards-Wilkinson fluctuations for 2-dimensional KPZ). Let $h^{\varepsilon}$ be the solution of the mollified $K P Z$ equation (1.3), with $\beta_{\varepsilon}$ as in (1.2) and $\hat{\beta} \in(0,1)$. Denote

$$
\mathfrak{h}^{\varepsilon}(t, x):=\frac{h^{\varepsilon}(t, x)-\mathbb{E}\left[h^{\varepsilon}(t, x)\right]}{\beta_{\varepsilon}}=\frac{\sqrt{\log \varepsilon^{-1}}}{\sqrt{2 \pi} \hat{\beta}}\left(h^{\varepsilon}(t, x)-\mathbb{E}\left[h^{\varepsilon}(t, x)\right]\right),
$$

where the centering satisfies $\mathbb{E}\left[h^{\varepsilon}(t, x)\right]=-\frac{1}{2} \sigma_{\hat{\beta}}^{2}+o(1)$ as $\varepsilon \downarrow 0$, see (1.6).

For any $t>0$ and $\phi \in C_{c}\left(\mathbb{R}^{2}\right)$, the following convergence in law holds:

$$
\left\langle\mathfrak{h}^{\varepsilon}(t, \cdot), \phi(\cdot)\right\rangle=\int_{\mathbb{R}^{2}} \mathfrak{h}^{\varepsilon}(t, x) \phi(x) \mathrm{d} x \underset{\varepsilon \downarrow 0}{\stackrel{d}{\longrightarrow}}\left\langle v^{\left(c_{\hat{\beta}}\right)}(t, \cdot), \phi(\cdot)\right\rangle,
$$

where $v^{(c)}(t, x)$ is the solution of the two-dimensional additive Stochastic Heat Equation

$$
\left\{\begin{array}{rl}
\partial_{t} v^{(c)}(t, x) & =\frac{1}{2} \Delta v^{(c)}(t, x)+c \xi(t, x) \\
v^{(c)}(0, x) & \equiv 0
\end{array}, \quad \text { where } c:=c_{\hat{\beta}}:=\sqrt{\frac{1}{1-\hat{\beta}^{2}}} .\right.
$$

Remark 1.2. For the version (1.7) of KPZ, Chatterjee and Dunlap showed in CD18 that any subsequential limit of $\widetilde{h}^{\varepsilon}-\mathbb{E}\left[\widetilde{h}^{\varepsilon}\right]$ as $\varepsilon \downarrow 0$ does not coincide with the solution of the additive SHE obtained by simply dropping the non-linearity $\beta_{\varepsilon}\left|\nabla \tilde{h}^{\varepsilon}\right|^{2}$ in (1.7). Here we show that the limit of $\tilde{h}^{\varepsilon}-\mathbb{E}\left[\tilde{h}^{\varepsilon}\right]$ actually coincides with the solution of the additive SHE with a strictly larger noise strength $c=c_{\hat{\beta}}>1$. In other words, the non-linearity in (1.7) produces an independent non-zero noise term in the limit, even though its strength $\beta_{\varepsilon} \rightarrow 0$.

Our proof of Theorem 1.1 is based on an analogous fluctuation result we proved in [CSZ17b] for the solution of the SHE (1.5). The independent noise can be seen to arise from the second and higher order chaos expansions of the solution, supported on microscopic scales.

Remark 1.3. We can view $\mathfrak{h}^{\varepsilon}(t, \cdot)$ as a random distribution on $\mathbb{R}^{2}$, i.e. a random element of the space of distributions $\mathcal{D}^{\prime}$, the dual space of $\mathcal{D}=C_{c}^{\infty}\left(\mathbb{R}^{2}\right)$. Our results show that $\mathfrak{h}^{\varepsilon}(t, \cdot)$ converges in law to $v^{\left(c_{\hat{\beta}}\right)}(t, \cdot)$ as random distributions. This is because convergence 
in law on $\mathcal{D}^{\prime}$ is equivalent to the pointwise convergence of the characteristic functional [F67, Th. III.6.5] (see also [BDW17, Cor. 2.4] for an analogue for tempered distributions):

$$
\forall \phi \in \mathcal{D}=C_{c}^{\infty}\left(\mathbb{R}^{2}\right): \quad \mathbb{E}\left[e^{i\left\langle\mathfrak{h}^{\varepsilon}(t, \cdot), \phi(\cdot)\right\rangle}\right] \underset{\varepsilon \downarrow 0}{\longrightarrow} \mathbb{E}\left[e^{i\left\langle v^{\left(c_{\hat{\beta}}\right)}(t, \cdot), \phi(\cdot)\right\rangle}\right]
$$

and this clearly follows by (1.10).

Remark 1.4. For simplicity, we only formulated the convergence of $\mathfrak{h}^{\varepsilon}(t, \cdot)$ to $v^{\left(c_{\hat{\beta}}\right)}(t, \cdot)$ as a random distribution in space for each fixed $t$. However, our proof can be easily adapted to prove the convergence of $\mathfrak{h}^{\varepsilon}(\cdot, \cdot)$ to $v^{\left(c_{\hat{\beta}}\right)}(\cdot, \cdot)$ as a random distribution in space and time.

Remark 1.5. The solution $v^{(c)}(t, \cdot)$ of the additive SHE (1.11), also known as the EdwardsWilkinson equation [EW82], is the random distribution on $\mathbb{R}^{2}$ formally given by

$$
v^{(c)}(t, x)=c \int_{0}^{t} \int_{\mathbb{R}^{2}} g_{t-s}(x-z) \xi(s, z) \mathrm{d} s \mathrm{~d} z, \quad \text { with } \quad g_{t}(x)=\frac{1}{2 \pi t} e^{-\frac{|x|^{2}}{2 t}} .
$$

For any $\phi \in C_{c}\left(\mathbb{R}^{2}\right)$, we have that $\left\langle v^{(c)}(t, \cdot), \phi\right\rangle:=\int_{\mathbb{R}^{2}} v^{(c)}(t, x) \phi(x) \mathrm{d} x$ is a Gaussian random variable with zero mean and variance

$$
\operatorname{Var}\left[\left\langle v^{(c)}(t, \cdot), \phi\right\rangle\right]=c^{2} \sigma_{\phi}^{2}, \quad \sigma_{\phi}^{2}:=\left\langle\phi, K_{t} \phi\right\rangle=\int_{\left(\mathbb{R}^{2}\right)^{2}} \phi(x) K_{t}(x, y) \phi(y) \mathrm{d} x \mathrm{~d} y,
$$

where the covariance kernel is given by

$$
K_{t}(x, y):=\int_{0}^{t} \frac{1}{4 \pi u} e^{-\frac{|x-y|^{2}}{4 u}} \mathrm{~d} u=\frac{1}{4 \pi} \int_{\frac{|x-y|^{2}}{4 t}}^{\infty} \frac{e^{-z}}{z} \mathrm{~d} z .
$$

In [CSZ17b] we also proved Edwards-Wilkinson fluctuations for the solution $u^{\varepsilon}$ of the 2-dimensional multiplicative SHE (1.5). More precisely, if similarly to (1.9) we set

$$
\mathfrak{u}^{\varepsilon}(t, x):=\frac{1}{\beta_{\varepsilon}}\left(u^{\varepsilon}(t, x)-\mathbb{E}\left[u^{\varepsilon}(t, x)\right]\right)=\frac{\sqrt{\log \varepsilon^{-1}}}{\sqrt{2 \pi} \hat{\beta}}\left(u^{\varepsilon}(t, x)-1\right),
$$

then as $\varepsilon \downarrow 0$ we have the convergence in law $\left\langle\mathfrak{u}^{\varepsilon}(t, \cdot), \phi(\cdot)\right\rangle \rightarrow\left\langle v^{\left(c_{\hat{\beta}}\right)}(t, \cdot), \phi(\cdot)\right\rangle$ as in (1.10) in the entire subcritical regime $\hat{\beta} \in(0,1)$, see [CSZ17b, Theorem 2.17] (which is formulated for space-time fluctuations, but its proof is easily adapted to space fluctuations).

Since $u^{\varepsilon}(t, x)=\exp \left(h^{\varepsilon}(t, x)\right)$, it is tempting to relate (1.15) and (1.9) via Taylor expansion. This is non obvious, because the one-point distributions of $h^{\varepsilon}(t, x)$ do not vanish as $\varepsilon \downarrow 0$, see (1.6), so we cannot approximate $h^{\varepsilon}(t, x) \approx u^{\varepsilon}(t, x)-1$. We will show in Section 2 that the approximation of $h(t, x)$ is highly non trivial, and the main contribution actually comes from specific parts of the expansion of $u(t, x)$ which are negligible relative to $u(t, x)$.

For future work, the goal will be to understand the scaling limit of the KPZ solution $h^{\varepsilon}(t, x)$ at or above the critical point $\hat{\beta}_{c}=1$. To our best knowledge, this remains a mystery also for physicists (even the weak to strong disorder transition (1.6) discovered in [CSZ17b] seems not to have been noted previously in the physics literature). Also the scaling limit of the SHE solution $u^{\varepsilon}(t, x)$ at or above the critical point is not completely known, even though we recently made some progress at the critical point [CSZ18], improving the study initiated in [BC98] (where the regime (1.2), with $\hat{\beta}$ close to 1 , was first studied).

We conclude this subsection with an overview of related results. In space dimension $d=1$, the Cole-Hopf solution $h(t, x):=\log u(t, x)$ of the KPZ equation (1.1) is well-defined as a random function, for any $\beta \in(0, \infty)$, and there is no phase transition in the one-point 
distribution as $\beta$ varies. Edwards-Wilkinson fluctuations for $h(t, x)$ and $u(t, x)$ are easily established as $\beta \downarrow 0$, combining Wiener chaos and Taylor expansion (because $u(t, x) \rightarrow 1$ ).

In space dimensions $d \geqslant 3$, the right way to scale the disorder strength is $\beta_{\varepsilon}=\hat{\beta} \varepsilon^{\frac{d-2}{2}}$. It was shown in [MSZ16, CCM18] that the mollified SHE solution $u^{\varepsilon}(t, x)$ of (1.5) undergoes a weak to stronger disorder transition, similar to the directed polymer model [CSY04]: there is a critical value $\hat{\beta}_{c} \in(0, \infty)$ such that $u^{\varepsilon}(t, x)$ converges in law as $\varepsilon \downarrow 0$ to a strictly positive limit when $\hat{\beta}<\hat{\beta}_{c}$, while it converges to zero if $\hat{\beta}>\hat{\beta}_{c}$. The KPZ solution $h^{\varepsilon}(t, x)=\log u^{\varepsilon}(t, x)$ is thus qualitatively similar to the 2-dimensional case (1.6): $h^{\varepsilon}(t, x)$ converges in law to a finite limit for $\hat{\beta}<\hat{\beta}_{c}$, while it converges to $-\infty$ for $\hat{\beta}>\hat{\beta}_{c}$. The value of $\hat{\beta}_{c}$ is unknown.

Edwards-Wilkinson fluctuations for the $\mathrm{KPZ}$ solution $h^{\varepsilon}(t, x)$ in dimension $d \geqslant 3$ have been established recently by Magnen and Unterberger [MU18], assuming that the noise strength $\hat{\beta}$ is sufficiently small. The corresponding result for the SHE solution $u^{\varepsilon}(t, x)$ was proved in [GRZ18, CCM18. The approaches in these papers do not allow to cover the entire subcritical regime, as we do in dimension 2 .

We should also mention that in space dimension $d=2$, Edwards-Wilkinson fluctuations are believed to hold (and verified in some cases, see e.g. [T17]) also for models in the anisotropic KPZ class, where anysotropy means that the term $|\nabla h|^{2}$ in the KPZ equation (1.1) is replaced by $\langle\nabla h, A \nabla h\rangle$ for some matrix $A$ with $\operatorname{det}(A) \leqslant 0$.

Shortly after we posted our paper, Dunlap et al. [DGRZ18] gave an alternative proof (to [MU18]) of Edwards-Wilkinson fluctuations for the KPZ equation in dimension $d \geqslant 3$ when $\hat{\beta}$ is sufficiently small. Using the same techniques (Clark-Ocone formula and second order Poincaré inequality), Gu G18] proved the same Edwards-Wilkinson fluctuation as in our Theorem 1.1 for the KPZ equation in dimension $d=2$, except his result is restricted to $\hat{\beta}$ small instead of covering the entire subcritical regime.

1.2. The Directed POlymer Model. In this subsection, we state our result for the partition function of the directed polymer model in dimension 2+1. See [C17] for an overview of the directed polymer model. In the language of disordered systems, space dimension 2 is critical for this model, where disorder is marginally relevant. For further background on the notion of disorder relevance/irrelevance (which corresponds to subcriticality/supercriticality in the context of singular SPDEs), see e.g. [H74, G10, CSZ17a].

The directed polymer model is defined as a change of measure for a random walk, depending on a random environment (disorder). Let $S$ be the simple symmetric random walk on $\mathbb{Z}^{2}$. If $S$ starts at $x \in \mathbb{Z}^{2}$, then we denote its law by $\mathrm{P}_{x}$ with expectation $\mathrm{E}_{x}$, and we omit $x$ when $x=0$. We set

$$
q_{n}(x):=\mathrm{P}\left(S_{n}=x\right) .
$$

Denoting by $\widetilde{S}$ an independent copy of $S$, we define the expected overlap by

$$
R_{N}:=\sum_{n=1}^{N} \mathrm{P}\left(S_{n}=\widetilde{S}_{n}\right)=\sum_{n=1}^{N} \sum_{x \in \mathbb{Z}^{2}} q_{n}(x)^{2}=\sum_{n=1}^{N} q_{2 n}(0)=\frac{\log N}{\pi}+O(1) .
$$

We fix $\hat{\beta} \in(0, \infty)$ and define $\left(\beta_{N}\right)_{N \in \mathbb{N}}$ by

$$
\beta_{N}:=\frac{\hat{\beta}}{\sqrt{R_{N}}}=\frac{\sqrt{\pi} \hat{\beta}}{\sqrt{\log N}}\left(1+\frac{O(1)}{\log N}\right) .
$$


Disorder is given by i.i.d. random variables $(\omega(n, x))_{n \in \mathbb{N}, x \in \mathbb{Z}^{2}}$ with law $\mathbb{P}$, such that

$$
\mathbb{E}[\omega]=0, \quad \mathbb{E}\left[\omega^{2}\right]=1, \quad \lambda(\beta):=\log \mathbb{E}\left[e^{\beta \omega}\right]<\infty \quad \forall \beta>0 \text { small enough } .
$$

For technical reasons, we require that the law of $\omega$ satisfies a concentration inequality. Recall that a function $f: \mathbb{R}^{n} \rightarrow \mathbb{R}$ is called 1-Lipschitz if $|f(x)-f(y)| \leqslant|x-y|$ for all $x, y \in \mathbb{R}^{n}$, with $|\cdot|$ the Euclidean norm. We assume the following:

$\exists \gamma>1, C_{1}, C_{2} \in(0, \infty):$ for all $n \in \mathbb{N}$ and $f: \mathbb{R}^{n} \rightarrow \mathbb{R}$ convex and 1-Lipschitz

$$
\mathbb{P}\left(\left|f\left(\omega_{1}, \ldots, \omega_{N}\right)-M_{f}\right| \geqslant t\right) \leqslant C_{1} \exp \left(-\frac{t^{\gamma}}{C_{2}}\right),
$$

where $M_{f}$ denotes a median of $f\left(\omega_{1}, \ldots, \omega_{N}\right)$. (By changing $C_{1}, C_{2}$, one can equivalently replace $M_{f}$ by $\mathbb{E}\left[f\left(\omega_{1}, \ldots, \omega_{N}\right)\right]$, see [Led01, Proposition 1.8].) Condition (1.20) is satisfied if $\omega$ is bounded, or if it is Gaussian, or more generally if it has a density $\exp (-V(\cdot)+U(\cdot))$, with $V$ uniformly strictly convex and $U$ bounded. See Led01 for more details.

Given $\omega, N \in \mathbb{N}$, and $\beta_{N}$ as defined in (1.18), we define the Hamiltonian by

$$
H_{N}:=\sum_{n=1}^{N}\left(\beta_{N} \omega\left(n, S_{n}\right)-\lambda\left(\beta_{N}\right)\right)=\sum_{n=1}^{N} \sum_{y \in \mathbb{Z}^{2}}\left(\beta_{N} \omega(n, y)-\lambda\left(\beta_{N}\right)\right) \mathbb{1}_{\left\{S_{n}=y\right\}} .
$$

We will be interested in the family of partition functions

$$
\begin{array}{lr}
Z_{N}(x)=Z_{N, \beta_{N}}(x)=\mathrm{E}_{x}\left[e^{H_{N}}\right], & N \in \mathbb{N}, x \in \mathbb{Z}^{2}, \\
Z_{N}(x):=Z_{\lfloor N\rfloor}(\lfloor x\rfloor), & N \in[0, \infty), x \in \mathbb{R}^{2} .
\end{array}
$$

We will write $Z_{N}:=Z_{N}(0)$ for simplicity. Note that the law of $Z_{N}(x)$ does not depend on $x \in \mathbb{Z}^{2}$, and we have $\mathbb{E}\left[Z_{N}(x)\right]=\mathbb{E}\left[Z_{N}\right]=1$.

The partition function $Z_{N}(x)$ is a discrete analogue (modulo a time reversal) of the SHE solution $u^{\varepsilon}(t, x)$ in (1.5), as can be seen from its Feynman-Kac formula (5.1) below (see also [AKQ14]). Then $\log Z_{N}(x)$ is a discrete analogue of the KPZ solution $h^{\varepsilon}(t, x)$ in (1.3). In fact, we proved in [CSZ17b, Theorem 2.8] that for $\beta_{N}$ as in (1.18), the random variable $\log Z_{N}(x)$ converges in distribution to the same limit as in (1.6), with critical value $\hat{\beta}_{c}=1$. It is not surprising that here we can also prove the following analogue of Theorem [1.1.

Theorem 1.6 (Edwards-Wilkinson fluctuations for directed polymer). Let $Z_{N, \beta_{N}}(x)$ be the family of partition functions defined as in (1.22), with $\beta_{N}$ as in (1.18) with $\hat{\beta} \in(0,1)$, and the disorder $\omega$ satisfying assumptions (1.19) and (1.20). Denote

$$
\mathfrak{h}_{N}(t, x):=\frac{\log Z_{t N}(x \sqrt{N})-\mathbb{E}\left[\log Z_{t N}\right]}{\beta_{N}}=\frac{\sqrt{\log N}}{\sqrt{\pi} \hat{\beta}}\left(\log Z_{t N}(x \sqrt{N})-\mathbb{E}\left[\log Z_{t N}\right]\right) .
$$

For any $t>0$ and $\phi \in C_{c}^{\infty}\left(\mathbb{R}^{2}\right)$, the following convergence in law holds, with $c_{\hat{\beta}}$ as in (1.11):

$$
\left\langle\mathfrak{h}_{N}(t, \cdot), \phi(\cdot)\right\rangle=\int_{\mathbb{R}^{2}} \mathfrak{h}_{N}(t, x) \phi(x) \mathrm{d} x \underset{N \rightarrow \infty}{\stackrel{d}{\longrightarrow}}\left\langle v^{\left(\sqrt{2} c_{\hat{\beta}}\right)}(t / 2, \cdot), \phi(\cdot)\right\rangle,
$$

where $v^{(c)}(s, x)$ is the solution of the two-dimensional additive SHE as in (1.11).

Remark 1.7. Here the limit $v^{\left(\sqrt{2} c_{\hat{\beta}}\right)}(t / 2, \cdot)$ differs from $v^{\left(c_{\hat{\beta}}\right)}(t, \cdot)$ in Theorem 1.1 because the increment of the simple symmetric random walk on $\mathbb{Z}^{2}$ has covariance matrix $\frac{1}{2} I$.

We will in fact prove Theorem 1.6 first, since the structure is more transparent in the discrete setting, and then outline the changes needed to prove Theorem 1.1 for KPZ. 
1.3. Outhine. The rest of the paper is organized as follows.

- In Section 2, we present the proof steps and describe the main ideas.

- In Section 3, we give bounds on positive and negative moments for the directed polymer partition function, based on concentration inequalities and hypercontractivity.

- In Section 4, we prove our main result Theorem 1.6 for directed polymer.

- In Section 5, we explain how the proof for directed polymer can be adapted to prove our main result Theorem 1.1 for KPZ.

We will conclude with a few appendices which might be of independent interest, where we prove some results needed in the proofs.

- Appendix A establishes scaling relations for KPZ with different parameters.

- Appendix B recalls and refines known hypercontractivity results for suitable functions (polynomial chaos) of i.i.d. random variables.

- Appendix [C formulates a concentration of measure result for the left tail of convex functions that are not globally Lipschitz, defined on general Gaussian spaces.

- Lastly in Appendix D we discuss linearity and measurability properties of stochastic integrals, which are needed in the proof in Section 5 .

\section{OUTLINE OF PROOF STEPS AND MAIN IDEAS}

In this section, we outline the proof steps for Theorems 1.1 and 1.6 and describe the basic setup. We focus on the directed polymer partition function (the case of KPZ follows the same steps). The two main ideas are a decomposition of the partition function $Z_{N}$ which allows to "linearize" $\log Z_{N}$ (see 2.1), and a representation of $Z_{N}$ as a polynomial chaos expansion in the disorder (see \$2.2). The "linearization" of $\log Z_{N}$ essentially reduces Theorem [1.6 to an analogous result for $Z_{N}$ which we proved in [CSZ17b, Theorem 2.13].

2.1. Decomposition and linearization. Given a subset $\Lambda \subseteq \mathbb{N} \times \mathbb{Z}^{2}$, we denote by $Z_{\Lambda, \beta}(x)$ the partition function where disorder is only sampled from within $\Lambda$, i.e.

$$
Z_{\Lambda, \beta}(x):=\mathrm{E}_{x}\left[e^{H_{\Lambda, \beta}}\right], \quad \text { where } \quad H_{\Lambda, \beta}:=\sum_{(n, x) \in \Lambda}\left(\beta \omega_{n, x}-\lambda(\beta)\right) \mathbb{1}_{\left\{S_{n}=x\right\}} .
$$

The original partition function $Z_{N, \beta}(x)$ in (1.21)-(1.22) corresponds to $\Lambda=\{1, \ldots, N\} \times \mathbb{Z}^{2}$.

In our previous study in CSZ17b], a key observation was that for $\hat{\beta} \in(0,1)$ the partition function $Z_{N, \beta_{N}}(x)$ essentially depends only on disorder in a space-time window around the starting point $(0, x)$ that is negligible on the diffusive scale $(N, \sqrt{N})$. This motivates us to approximate $Z_{N, \beta_{N}}(x)$ by a partition function $Z_{N, \beta_{N}}^{A}(x)$ with disorder present only in such a space-time window $A_{N}^{x}$. More precisely, we define a scale parameter $a_{N}$ tending to zero as

$$
a_{N}:=\frac{1}{(\log N)^{1-\gamma}} \quad \text { with } \quad \gamma \in\left(0, \gamma^{*}\right)
$$

where $\gamma^{*}>0$ depends only on $\hat{\beta}$ in Theorem 1.6 and its choice will be clear from the estimate in (4.4) later on. We now introduce the space-time window

$$
A_{N}^{x}:=\left\{(n, z) \in \mathbb{N} \times \mathbb{Z}^{2}: n \leqslant N^{1-a_{N}},|z-x|<N^{\frac{1}{2}-\frac{a_{N}}{4}}\right\},
$$


and define $Z_{N, \beta}^{A}(x)$ as the partition function which only samples disorder in $A_{N}^{x}$, i.e.

$$
Z_{N, \beta}^{A}(x):=Z_{\Lambda, \beta}(x) \text { with } \Lambda=A_{N}^{x} .
$$

We then decompose the original partition function $Z_{N, \beta}(x)$ as follows:

$$
Z_{N, \beta}(x)=Z_{N, \beta}^{A}(x)+\hat{Z}_{N, \beta}^{A}(x),
$$

where $\hat{Z}_{N, \beta}^{A}(x)$, defined by the previous relation, is a "remainder". In a sense that we will make precise later (see (3.4) ) it holds that for any fixed $x, \hat{Z}_{N, \beta_{N}}^{A}(x) \ll Z_{N, \beta_{N}}^{A}(x)$ and thus

$$
\log Z_{N, \beta_{N}}(x)=\log Z_{N, \beta_{N}}^{A}(x)+\log \left(1+\frac{\hat{Z}_{N, \beta_{N}}^{A}(x)}{Z_{N, \beta_{N}}^{A}(x)}\right) \approx \log Z_{N, \beta_{N}}^{A}(x)+\frac{\hat{Z}_{N, \beta_{N}}^{A}(x)}{Z_{N, \beta_{N}}^{A}(x)} .
$$

More precisely, if we define the error $O_{N}(x)$ via

$$
\log Z_{N, \beta_{N}}(x)=\log Z_{N, \beta_{N}}^{A}(x)+\frac{\hat{Z}_{N, \beta_{N}}^{A}(x)}{Z_{N, \beta_{N}}^{A}(x)}+O_{N}(x),
$$

then we will show the following:

Proposition 2.1. Let $O_{N}(\cdot)$ be defined as in (2.7), then for any $\phi \in C_{c}\left(\mathbb{R}^{2}\right)$

$$
\sqrt{\log N} \frac{1}{N} \sum_{x \in \mathbb{Z}^{2}}\left(O_{N}(x)-\mathbb{E}\left[O_{N}(x)\right]\right) \phi\left(\frac{x}{\sqrt{N}}\right) \underset{N \rightarrow \infty}{\stackrel{L^{2}(\mathbb{P})}{\longrightarrow}} 0 .
$$

Remarkably, even though $\log Z_{N, \beta_{N}}^{A}(x)$ gives the dominant contribution to $\log Z_{N, \beta_{N}}(x)$ for any fixed $x$, it does not contribute to the fluctuations of $\log Z_{N, \beta_{N}}(x)$ when averaged over $x$, that is:

Proposition 2.2. Let $Z_{N, \beta_{N}}^{A}(\cdot)$ be defined as in (2.4), then for any $\phi \in C_{c}\left(\mathbb{R}^{2}\right)$

$$
\sqrt{\log N} \frac{1}{N} \sum_{x \in \mathbb{Z}^{2}}\left(\log Z_{N, \beta_{N}}^{A}(x)-\mathbb{E}\left[\log Z_{N, \beta_{N}}^{A}(x)\right]\right) \phi\left(\frac{x}{\sqrt{N}}\right) \underset{N \rightarrow \infty}{\stackrel{L^{2}(\mathbb{P})}{\longrightarrow}} 0 .
$$

As a consequence, the fluctuations of $\log Z_{N, \beta_{N}}(\cdot)$ are determined by the "normalized remainder" $\hat{Z}_{N, \beta_{N}}^{A}(\cdot) / Z_{N, \beta_{N}}^{A}(\cdot)$. To determine the fluctuations of this term, we define the set

$$
B_{N}^{\geqslant}:=\left(\left(N^{1-9 a_{N} / 40}, N\right] \cap \mathbb{N}\right) \times \mathbb{Z}^{2},
$$

and we let $Z_{N, \beta_{N}}^{B \geqslant}(x)$ be the partition function where disorder is sampled only from $B_{N}^{\geqslant}$, i.e.

$$
Z_{N, \beta_{N}}^{B \geqslant}(x):=Z_{\Lambda, \beta_{N}}(x) \text { with } \Lambda=B_{N}^{\geqslant} .
$$

Note that $\mathbb{E}\left[Z_{N, \beta_{N}}^{B \geqslant}(x)\right]=1$, so $\left(Z_{N, \beta_{N}}^{B \geqslant}(x)-1\right)$ is a centered random variable. The key point, and the more involved step, will be to show that

in the following sense.

$$
\hat{Z}_{N, \beta_{N}}^{A}(x) \approx Z_{N, \beta_{N}}^{A}(x)\left(Z_{N, \beta_{N}}^{B \geqslant}(x)-1\right),
$$

Proposition 2.3. Let $Z_{N, \beta_{N}}^{A}(\cdot), \hat{Z}_{N, \beta_{N}}^{A}(\cdot), Z_{N, \beta_{N}}^{B \geqslant}(\cdot)$ be defined as in (2.4), (2.5), (2.11). Then for any $\phi \in C_{c}\left(\mathbb{R}^{2}\right)$

$$
\sqrt{\log N} \frac{1}{N} \sum_{x \in \mathbb{Z}^{2}}\left(\frac{\hat{Z}_{N, \beta_{N}}^{A}(x)}{Z_{N, \beta_{N}}^{A}(x)}-\left(Z_{N, \beta_{N}}^{B \geqslant}(x)-1\right)\right) \phi\left(\frac{x}{\sqrt{N}}\right) \underset{N \rightarrow \infty}{\stackrel{L^{1}(\mathbb{P})}{\longrightarrow}} 0 .
$$


It remains to identify the fluctuations of $Z_{N, \beta_{N}}^{B \geqslant}(\cdot)$. This falls within the scope of Theorem 2.13 in [CSZ17b], which we will recall in Section 4.4 and which will show that the fluctuations of $Z_{N, \beta_{N}}^{B \geqslant}(\cdot)$ converges to the solution $v^{\left(\sqrt{2} c_{\hat{\beta}}\right)}(1 / 2, \cdot)$ of the two-dimensional additive SHE, as in Theorem 1.6. The proof is based on polynomial chaos expansions of the partition function, which we will recall in the next subsection.

Proposition 2.4. Let $Z_{N, \beta_{N}}^{B \geqslant}(\cdot)$ be defined as in (2.11). Then

$$
\frac{\sqrt{\log N}}{\sqrt{\pi} \hat{\beta}} \frac{1}{N} \sum_{x \in \mathbb{Z}^{2}}\left(Z_{N, \beta_{N}}^{B \geqslant}(x)-1\right) \phi\left(\frac{x}{\sqrt{N}}\right) \underset{N \rightarrow \infty}{\stackrel{d}{\longrightarrow}}\left\langle v^{\left(\sqrt{2} c_{\hat{\beta}}\right)}(1 / 2, \cdot), \phi\right\rangle,
$$

where $v^{(c)}(s, x)$ is the solution of the two-dimensional additive SHE as in (1.11).

Theorem 1.6 is a direct corollary of the decomposition (2.7) and Propositions 2.1 2.4, Regarding the centering, it suffices to note that $\mathbb{E}\left[\log Z_{N, \beta_{N}}(x)\right]=\mathbb{E}\left[\log Z_{N, \beta_{N}}^{A}(x)\right]+\mathbb{E}\left[O_{N}(x)\right]$, because the random variable $\hat{Z}_{N, \beta_{N}}^{A}(x) / Z_{N, \beta_{N}}^{A}(x)$ has zero mean, which follows from the polynomial chaos expansions of partition functions that we now present.

2.2. Polynomial Chaos expansions. Our analysis of the partition functions $Z_{N, \beta_{N}}^{A}(\cdot)$, $\hat{Z}_{N, \beta_{N}}^{A}(\cdot), Z_{N, \beta_{N}}^{B}(\cdot)$ is based on multi-linear expansions, known as polynomial chaos expansions, which have also been used extensively in [CSZ17a, CSZ17b].

Recall the definition (1.18) of $\beta_{N}$ and our assumptions (1.19) on the disorder, and note that by (1.19) and Taylor expansion, $\lambda(2 \beta)-2 \lambda(\beta) \sim \beta^{2}$ as $\beta \rightarrow 0$. We introduce the sequence

$$
\sigma_{N}:=\sqrt{e^{\lambda\left(2 \beta_{N}\right)-2 \lambda\left(\beta_{N}\right)}-1} \underset{N \rightarrow \infty}{\sim} \beta_{N}
$$

where we agree that $a_{N} \sim b_{N}$ means $\lim _{N \rightarrow \infty} a_{N} / b_{N}=1$, and we define the random variables

$$
\xi_{n, x}^{(N)}:=\sigma_{N}^{-1}\left(e^{\beta_{N} \omega(n, x)-\lambda\left(\beta_{N}\right)}-1\right) .
$$

We will suppress the dependence of $\xi_{n, x}^{(N)}$ on $N$, for notational simplicity. Note that $\left(\xi_{n, x}\right)_{n \in \mathbb{N}, x \in \mathbb{Z}^{2}}$ are i.i.d. with $\mathbb{E}\left[\xi_{n, x}\right]=0$ and $\mathbb{E}\left[\xi_{n, x}^{2}\right]=1$.

Recall the definition (1.21)-(1.22) of the partition function $Z_{N, \beta_{N}}(x)$ of the polymer that starts at time zero from location $x$. This can be written as

$$
\begin{aligned}
Z_{N, \beta_{N}}(x) & =\mathrm{E}_{x}\left[\prod_{1 \leqslant n \leqslant N, y \in \mathbb{Z}^{2}}\left(1+\sigma_{N} \xi_{n, y} \mathbb{1}_{\left\{S_{n}=y\right\}}\right)\right] \\
& =1+\sum_{k=1}^{N} \sigma_{N}^{k} \sum_{\substack{0=n_{0}<n_{1}<\ldots<n_{k} \leqslant N \\
x_{0}=x, x_{1}, \ldots, x_{k} \in \mathbb{Z}^{2}}} \prod_{i=1}^{k} q_{n_{i}-n_{i-1}}\left(x_{i}-x_{i-1}\right) \xi_{n_{i}, x_{i}},
\end{aligned}
$$

where $q_{n}(x):=\mathrm{P}\left(S_{n}=x\right)$. Note that the terms in the sum are orthogonal to each other in $L^{2}$, and when $\hat{\beta} \in(0,1)$ the dominant contribution to $Z_{N, \beta_{N}}(x)$ comes from disorder $\xi$,. in a space-time window that is negligible on the diffusive scale. More precisely, a second moment calculation (see (3.4) below) shows that $Z_{N, \beta_{N}}(x)$ is close in $L^{2}$ to the partition function $Z_{N, \beta_{N}}^{A}(x)$ which only samples disorder from within $A_{N}^{x}$ (recall (2.3) $)$.

It will be convenient to introduce a concise representation for the expansion (2.17) as follows: given a point $\left(n_{0}, x_{0}\right)$ and a finite subset $\tau:=\left\{\left(n_{1}, x_{1}\right), \ldots,\left(n_{|\tau|}, x_{|\tau|}\right)\right\}$ of $\mathbb{N}_{0} \times \mathbb{Z}^{2}$ 


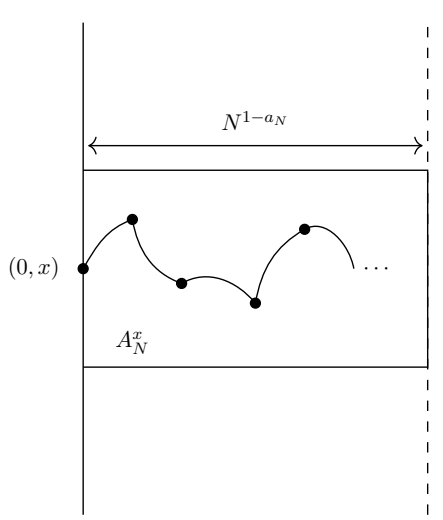

(A) Partition fuction $Z_{N, \beta_{N}}^{A}(x)$.

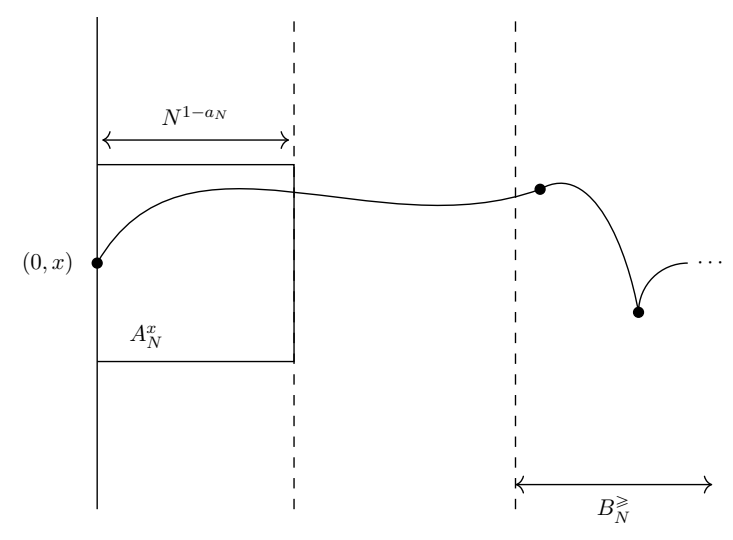

(B) Partition function $Z_{N, \beta_{N}}^{B \geqslant}(x)$.

Figure 1. The above figures depict the chaos expansions of $Z_{N, \beta_{N}}^{A}(x)$ and $Z_{N, \beta_{N}}^{B \geqslant}(x)$. The disorder sampled by $Z_{N, \beta_{N}}^{A}(x)$ is restricted to the set $A_{N}^{x}$, while that of $Z_{N, \beta_{N}}^{B \geqslant}(x)$ is restricted to $B_{N}^{\geqslant}$

with $n_{0}<n_{1}<\cdots<n_{|\tau|}$, we introduce the notation

$$
\boldsymbol{q}^{\left(n_{0}, x_{0}\right)}(\tau):=\prod_{i=1}^{|\tau|} q_{n_{i}-n_{i-1}}\left(x_{i}-x_{i-1}\right) \quad \text { and } \quad \boldsymbol{\xi}(\tau):=\prod_{i=1}^{|\tau|} \xi_{n_{i}, x_{i}} .
$$

For $\tau=\varnothing$ we define $\boldsymbol{q}^{\left(n_{0}, x\right)}(\tau)=\boldsymbol{\xi}(\tau):=1$. In this way we can write concisely the chaos expansion of $Z_{N, \beta_{N}}(x)$ as

$$
Z_{N, \beta_{N}}(x)=\sum_{\tau \subset\{1, \ldots, N\} \times \mathbb{Z}^{2}} \sigma_{N}^{|\tau|} \boldsymbol{q}^{(0, x)}(\tau) \boldsymbol{\xi}(\tau) .
$$

Similarly, for the partition functions $Z_{N, \beta}^{A}(x), Z_{N, \beta}^{B \geqslant}(x)$ in (2.4), (2.11) we can write

$$
Z_{N, \beta_{N}}^{A}(x)=\sum_{\tau \subset A_{N}^{x}} \sigma_{N}^{|\tau|} \boldsymbol{q}^{(0, x)}(\tau) \boldsymbol{\xi}(\tau), \quad Z_{N, \beta_{N}}^{B \geqslant}(x)=\sum_{\tau \subset B_{N}^{\geq}} \sigma_{N}^{|\tau|} \boldsymbol{q}^{(0, x)}(\tau) \boldsymbol{\xi}(\tau) .
$$

A graphical illustration of $Z_{N, \beta}^{A}(x)$ and $Z_{N, \beta}^{B \geqslant}(x)$ appears in Figure 1 .

These polynomial chaos expansions are discrete analogues of Wiener-Itô chaos expansions. They are especially suited for variance calculations and provide important insight. For instance, the partition function $\hat{Z}_{N, \beta}^{A}(x):=Z_{N, \beta}(x)-Z_{N, \beta}^{A}(x)$, see (2.5), is obtained by restricting the sum in (2.18) to $\tau$ which include space-time points outside the set $A_{N}^{x}$, and hence $\hat{Z}_{N, \beta_{N}}^{A}(x) / Z_{N, \beta_{N}}^{A}(x)$ has zero mean due to the independence between the disorder inside and outside $A_{N}^{x}$. Similarly, the centered partition function $\left(Z_{N, \beta}^{B \geqslant}(x)-1\right)$, which appears in (2.13)-(2.14), is the contribution to (2.19) given by configurations $\tau$ that contain only points (and at least one point) in $B_{N}^{\gtrless}$.

\section{MOMENT BOUndS}

In this section we collect some moment bounds that will be used in the proof. 
3.1. SeCOND moment. We bound the second moment of $Z_{N, \beta_{N}}(x), Z_{N, \beta_{N}}^{A}(x), \hat{Z}_{N, \beta_{N}}^{A}(x)$.

We start from $Z_{N, \beta_{N}}(x)$. It follows by (2.17) and (1.17) that

$$
\begin{aligned}
\mathbb{E}\left[Z_{N, \beta_{N}}(x)^{2}\right] & =\sum_{\tau \subset\{1, \ldots, N\} \times \mathbb{Z}^{2}}\left(\sigma_{N}^{2}\right)^{|\tau|} \boldsymbol{q}^{(0, x)}(\tau)^{2} \\
& =1+\sum_{k=1}^{N}\left(\sigma_{N}^{2}\right)^{k} \sum_{\substack{\sum_{0=: n_{0}<n_{1}<\ldots<n_{k} \leqslant N} \\
x_{0}=: x, x_{1}, \ldots, x_{k} \in \mathbb{Z}^{2}}} \prod_{i=1}^{k} q_{n_{i}-n_{i-1}}\left(x_{i}-x_{i-1}\right)^{2} \\
& =1+\sum_{k=1}^{N}\left(\sigma_{N}^{2}\right)^{k} \sum_{0=: n_{0}<n_{1}<\ldots<n_{k} \leqslant N} q_{2\left(n_{i}-n_{i-1}\right)}(0) .
\end{aligned}
$$

If we let each increment $n_{i}-n_{i-1}$ vary freely in $\{1,2, \ldots, N\}$, by (1.17) we get the bound $\mathbb{E}\left[Z_{N, \beta_{N}}(x)^{2}\right] \leqslant \sum_{k \geqslant 0}\left(\sigma_{N}^{2} R_{N}\right)^{k}=\left(1-\sigma_{N}^{2} R_{N}\right)^{-1}$. Recalling (1.18) and (2.15), we obtain

$$
\forall \hat{\beta} \in(0,1) \exists C_{\hat{\beta}}<\infty \text { such that } \forall N \in \mathbb{N}: \quad \mathbb{E}\left[Z_{N, \beta_{N}}(x)^{2}\right] \leqslant C_{\hat{\beta}},
$$

where $C_{\hat{\beta}}$ will denote a generic constant depending on $\hat{\beta}$.

Next we look at $Z_{N, \beta_{N}}^{A}(x)$. The polynomial chaos expansion for $Z_{N, \beta_{N}}^{A}(x)$ is a subset of the one for $Z_{N, \beta_{N}}(x)$, hence the same bound (3.2) applies:

$$
\forall \hat{\beta} \in(0,1) \exists C_{\hat{\beta}<\infty} \text { such that } \forall N \in \mathbb{N}: \quad \mathbb{E}\left[Z_{N, \beta_{N}}^{A}(x)^{2}\right] \leqslant C_{\hat{\beta}} .
$$

We turn to $\hat{Z}_{N, \beta_{N}}^{A}(x)$. The bound (3.2) can again be applied, but it is quite poor. In fact, the following much better bound holds (recall that $a_{N}$ is defined in (2.2)):

$$
\forall \hat{\beta} \in(0,1) \exists C_{\hat{\beta}}<\infty \text { such that } \forall N \in \mathbb{N}: \quad \mathbb{E}\left[\hat{Z}_{N, \beta_{N}}^{A}(x)^{2}\right] \leqslant C_{\hat{\beta}} a_{N} .
$$

The proof, given below, is elementary but slightly technical (see Subsection 3.4).

We conclude with an alternative viewpoint on the bound (3.2). If we denote by $S$ and $\widetilde{S}$ two independent copies of the random walk, by (1.21)-(1.22) we can compute

$$
\mathbb{E}\left[Z_{N, \beta_{N}}^{2}\right]=\mathbb{E} \mathrm{E}\left[e^{H_{N, \beta_{N}}(S)+H_{N, \beta_{N}}(\widetilde{S})}\right]=\mathrm{E}\left[e^{\left(\lambda\left(2 \beta_{N}\right)-2 \lambda\left(\beta_{N}\right)\right) \mathcal{L}_{N}(S, \widetilde{S})}\right],
$$

where $\mathcal{L}_{N}(S, \widetilde{S})$ is the overlap of the two copies $S, \widetilde{S}$ up to time $N$, defined by

$$
\mathcal{L}_{N}(S, \widetilde{S}):=\sum_{n=1}^{N} \mathbb{1}_{\left\{S_{n}=\widetilde{S}_{n}\right\}}=\left|S \cap \widetilde{S} \cap\left(\{1, \ldots, N\} \times \mathbb{Z}^{2}\right)\right| .
$$

Since $\lambda(\beta) \sim \frac{1}{2} \beta^{2}$ as $\beta \downarrow 0$, see (1.19), we get

$$
\mathbb{E}\left[Z_{N, \beta_{N}}^{2}\right]=\mathrm{E}\left[e^{\left(1+\varepsilon_{N}\right) \beta_{N}^{2} \mathcal{L}_{N}(S, \widetilde{S})}\right], \quad \text { where } \quad \lim _{N \rightarrow \infty} \varepsilon_{N}=0 .
$$

Note that $\frac{\pi}{\log N} \mathcal{L}_{N}(S, \widetilde{S})$ converges in law to a mean 1 exponential random variable, see e.g. [ET60]. This matches with $\lim _{N \rightarrow \infty} \mathbb{E}\left[Z_{N, \beta_{N}}^{2}\right]=\left(1-\hat{\beta}^{2}\right)^{-1}$ for $\beta_{N}$ as in (1.18). 
3.2. Positive moments via hypercontractivity. We will bound higher positive moments of our partition functions using the hypercontractivity of polynomial chaos [MOO10, which we recall (with some strengthening) in Appendix B.

By (2.17), each partition function $Z_{N, \beta_{N}}(x), Z_{N, \beta_{N}}^{A}(x), \hat{Z}_{N, \beta_{N}}^{A}(x)$ can be expressed as a series

$$
\sum_{k=0}^{\infty} X_{k}^{(N)}
$$

(actually a finite sum) where $X_{k}^{(N)}$ is a multi-linear polynomial of degree $k$ in the i.i.d.

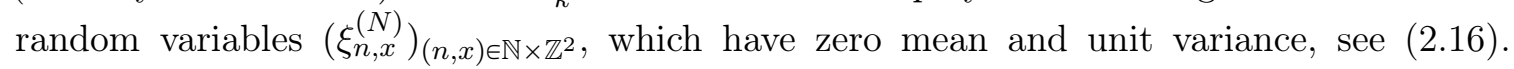
These random variables have uniformly bounded higher moments:

$$
\forall p \in(2, \infty): \quad \sup _{N \in \mathbb{N}} \mathbb{E}\left[\left|\xi_{n, x}^{(N)}\right|^{p}\right]<\infty,
$$

as one can check directly from (2.16) and (1.19) (see [CSZ17a, eq. (6.7)]).

Under these conditions, hypercontractivity ensures that, for every $p \in(2, \infty)$, the $p$-th moment of the series (3.8) can be bounded in terms of second moments:

$$
\mathbb{E}\left[\left|\sum_{k=0}^{\infty} X_{k}^{(N)}\right|^{p}\right] \leqslant\left(\sum_{k=0}^{\infty}\left(c_{p}^{k}\right)^{2} \mathbb{E}\left[\left(X_{k}^{(N)}\right)^{2}\right]\right)^{p / 2},
$$

where $c_{p} \in(1, \infty)$ is a constant, uniform in $N$, which only depends on the laws of the $\xi_{n, x}^{(N)}$. This is proved in [MOO10, $\S 3.2$ ] (extending [J97]), where a non-optimal value of $c_{p}$ is given. We will recall these results in Appendix $\mathrm{B}$, where we will prove that the optimal $c_{p}$ satisfies

$$
\lim _{p \downarrow 2} c_{p}=1 .
$$

This result, which is of independent interest, is crucial in order to apply (3.10) to our partition functions $Z_{N, \beta_{N}}(x), Z_{N, \beta_{N}}^{A}(x), \hat{Z}_{N, \beta_{N}}^{A}(x)$, because for any subcritical $\hat{\beta}<1$ we can fix $p>2$ such that $c_{p} \hat{\beta}<1$ is still subcritical. More precisely, note that multiplying $X_{k}^{(N)}$ by $c_{p}^{k}$ amounts to replacing $\sigma_{N}$ by $c_{p} \sigma_{N}$, see (2.17), and this corresponds asymptotically to replacing $\hat{\beta}$ by $c_{p} \hat{\beta}$, see (2.15) and (1.18). Then, by (3.2)-(3.4), we obtain:

$$
\begin{aligned}
& \forall \hat{\beta} \in(0,1) \quad \exists p=p_{\hat{\beta}} \in(2, \infty) \quad \exists C_{\hat{\beta}}^{\prime}<\infty \text { such that } \forall N \in \mathbb{N} \\
& \mathbb{E}\left[Z_{N, \beta_{N}}(x)^{p}\right] \leqslant C_{\hat{\beta}}^{\prime}, \quad \mathbb{E}\left[Z_{N, \beta_{N}}^{A}(x)^{p}\right] \leqslant C_{\hat{\beta}}^{\prime}, \quad \mathbb{E}\left[\left|\hat{Z}_{N, \beta_{N}}^{A}(x)\right|^{p}\right] \leqslant C_{\hat{\beta}}^{\prime}\left(a_{N}\right)^{p / 2} .
\end{aligned}
$$

3.3. Negative moments via Concentration. We give bounds on the negative moments of partition functions $Z_{N, \beta_{N}}(x)$ and $Z_{N, \beta_{N}}^{A}(x)$ (see (1.22), (2.17) and (2.3), (2.19)). We work with the general partition function $Z_{\Lambda, \beta}(x)$ defined in (2.1), which coincides with $Z_{N, \beta_{N}}(x)$, resp. $Z_{N, \beta_{N}}^{A}(x)$, for $\Lambda=\{1, \ldots, N\} \times \mathbb{Z}^{2}$, resp. $\Lambda=A_{N}^{x}$.

For fixed (say bounded) $\Lambda \subseteq \mathbb{N} \times \mathbb{Z}^{2}$, it is not difficult to show that the log partition function $\log Z_{\Lambda, \beta}$ is a convex and Lipschitz function of the random variables $(\omega(n, y)$ : $(n, y) \in \Lambda)$. However, if $\beta=\beta_{N}$ and the subset $\Lambda$ grows with $N$, its Lipschitz constant can diverge as $N \rightarrow \infty$, hence we cannot directly apply the concentration inequality (1.20). However, it turns out that, for any $\Lambda=\Lambda_{N} \subseteq\{1, \ldots, N\} \times \mathbb{Z}^{2}$, the Lipschitz constant is tight as $N \rightarrow \infty$. This yields the following estimate for the left tail of $\log Z_{\Lambda_{N}, \beta_{N}}$, proved below. 
Proposition 3.1 (Left tail). For any $\hat{\beta} \in(0,1)$, there exists $c_{\hat{\beta}} \in(0, \infty)$ with the following property: for every $N \in \mathbb{N}$ and for every choice of $\Lambda \subseteq\{1, \ldots, N\} \times \mathbb{Z}^{2}$, one has

$$
\forall t \geqslant 0: \quad \mathbb{P}\left(\log Z_{\Lambda, \beta_{N}} \leqslant-t\right) \leqslant c_{\hat{\beta}} e^{-t^{\gamma} / c_{\hat{\beta}}},
$$

where $\gamma>1$ is the same exponent appearing in assumption (1.20).

As a corollary, for every $p \in(0, \infty)$ we can estimate, uniformly in $\Lambda \subseteq\{1, \ldots, N\} \times \mathbb{Z}^{2}$,

$$
\begin{aligned}
\mathbb{E}\left[\left(Z_{\Lambda, \beta_{N}}\right)^{-p}\right] & =\mathbb{E}\left[e^{\left.-p \log Z_{\Lambda, \beta_{N}}\right]}=p \int_{-\infty}^{\infty} e^{p t} \mathbb{E}\left[\mathbb{1}_{\left\{t<-\log Z_{\Lambda, \beta_{N}}\right\}}\right] \mathrm{d} t\right. \\
& \leqslant 1+p \int_{0}^{\infty} e^{p t} c_{\hat{\beta}} \exp \left(-t^{\gamma} / c_{\hat{\beta}}\right) \mathrm{d} t=: C_{p, \hat{\beta}}<\infty .
\end{aligned}
$$

Choosing $\Lambda=\{1, \ldots, N\} \times \mathbb{Z}^{2}$ or $\Lambda=A_{N}^{x}$, we finally obtain the bounds

$$
\begin{aligned}
\forall \hat{\beta} \in(0,1) \forall p \in(0, \infty) \quad \exists C_{p, \hat{\beta}}<\infty: \quad & \sup _{N \in \mathbb{N}} \mathbb{E}\left[Z_{N, \beta_{N}}(x)^{-p}\right] \leqslant C_{p, \hat{\beta}}<\infty, \\
& \sup _{N \in \mathbb{N}} \mathbb{E}\left[Z_{N, \beta_{N}}^{A}(x)^{-p}\right] \leqslant C_{p, \hat{\beta}}<\infty .
\end{aligned}
$$

For later use, let us also state the following consequence:

$$
\forall \hat{\beta} \in(0,1) \forall p \in(0, \infty) \quad \exists C_{p, \hat{\beta}}<\infty: \quad \sup _{N \in \mathbb{N}} \mathbb{E}\left[\left|\log Z_{N, \beta_{N}}^{A}(x)\right|^{p}\right] \leqslant C_{p, \hat{\beta}}<\infty .
$$

The proof of this fact is simple: we can bound $|\log y| \leqslant C_{p}\left(y^{1 / p}+y^{-1 / p}\right)$ for all $y>0$ and for suitable $C_{p}<\infty$ (just distinguish $y \geqslant 1$ and $y<1$ ). This leads to $\mathbb{E}\left[\left|\log Z_{N, \beta_{N}}^{A}(x)\right|^{p}\right] \leqslant$ $C_{p}\left(\mathbb{E}\left[Z_{N, \beta_{N}}^{A}(x)\right]+\mathbb{E}\left[Z_{N, \beta_{N}}^{A}(x)^{-1}\right]\right)=C_{p}\left(1+\mathbb{E}\left[Z_{N, \beta_{N}}^{A}(x)^{-1}\right]\right)$, so (3.16) follows by (3.15).

It remains to prove Proposition 3.1. To this goal, we follow the strategy developed in [CTT17 for the pinning model, which generalizes [Mor14. We need the following result, which is [CTT17, Proposition 3.4], inspired by [Led01, Proposition 1.6].

Proposition 3.2. Assume that disorder $\omega$ has the concentration property (1.20). There exist constants $c_{1}, c_{2} \in(0, \infty)$ such that, for every $n \in \mathbb{N}$ and for every differentiable convex function $f: \mathbb{R}^{n} \rightarrow \mathbb{R}$, the following bound holds for all $a \in \mathbb{R}$ and $t, c \in(0, \infty)$,

$$
\mathbb{P}(f(\omega) \leqslant a-t) \mathbb{P}(f(\omega) \geqslant a,|\nabla f(\omega)| \leqslant c) \leqslant c_{1} \exp \left(-\frac{(t / c)^{\gamma}}{c_{2}}\right),
$$

where $\omega=\left(\omega_{1}, \ldots, \omega_{n}\right)$ and $|\nabla f(\omega)|:=\sqrt{\sum_{i=1}^{n}\left(\partial_{i} f(\omega)\right)^{2}}$ is the norm of the gradient.

We can deduce the bound (3.13) from (3.17) applied to the function $f=f_{N}$ given by

$$
f_{N}(\omega)=\log Z_{\Lambda, \beta_{N}} .
$$

We only need to bound from below the second probability in the left hand side of (3.17). This is provided by the next lemma, which completes the proof of Proposition 3.1.

Lemma 3.3. For any $\hat{\beta} \in(0,1)$, there exist $c_{\hat{\beta}} \in(0, \infty)$ and $\vartheta_{\hat{\beta}} \in(0,1)$ such that

$$
\inf _{N \in \mathbb{N}} \inf _{\Lambda \subseteq\{1, \ldots, N\} \times \mathbb{Z}^{2}} \mathbb{P}\left(f_{N}(\omega) \geqslant-\log 2,\left|\nabla f_{N}(\omega)\right| \leqslant c_{\hat{\beta}}\right) \geqslant \vartheta_{\hat{\beta}}>0 .
$$

Proof. We set $a=-\log 2$. For any $c>0$, we have

$$
\mathbb{P}\left(f_{N}(\omega) \geqslant a,\left|\nabla f_{N}(\omega)\right| \leqslant c\right)=\mathbb{P}\left(f_{N}(\omega) \geqslant a\right)-\mathbb{P}\left(f_{N}(\omega) \geqslant a,\left|\nabla f_{N}(\omega)\right|>c\right)
$$


The first probability can be estimated using the Paley-Zygmund inequality:

$$
\mathbb{P}\left(f_{N}(\omega) \geqslant a\right)=\mathbb{P}\left(Z_{\Lambda, \beta_{N}} \geqslant \frac{1}{2}\right)=\mathbb{P}\left(Z_{\Lambda, \beta_{N}} \geqslant \frac{1}{2} \mathbb{E}\left[Z_{\Lambda, \beta_{N}}\right]\right) \geqslant \frac{\mathbb{E}\left[Z_{\Lambda, \beta_{N}}\right]^{2}}{4 \mathbb{E}\left[\left(Z_{\Lambda, \beta_{N}}\right)^{2}\right]} .
$$

Note that $\mathbb{E}\left[Z_{\Lambda, \beta_{N}}\right]=1$. For $\Lambda \subseteq\{1, \ldots, N\} \times \mathbb{Z}^{2}$ we have $\mathbb{E}\left[\left(Z_{\Lambda, \beta_{N}}\right)^{2}\right] \leqslant \mathbb{E}\left[\left(Z_{N, \beta_{N}}\right)^{2}\right] \leqslant C_{\hat{\beta}}$, see (3.2), hence

$$
\mathbb{P}\left(f_{N}(\omega) \geqslant a\right) \geqslant \frac{1}{4 C_{\hat{\beta}}}=: 2 \vartheta_{\hat{\beta}} .
$$

We now proceed to estimate the second term in (3.20). First, we compute for $n \in \mathbb{N}, x \in \mathbb{Z}^{2}$

$$
\begin{gathered}
\frac{\partial f_{N}(\omega)}{\partial \omega_{n, x}}=\frac{1}{Z_{\Lambda, \beta_{N}}} \mathrm{E}\left[\beta_{N} \mathbb{1}_{(n, x) \in S \cap \Lambda} e^{H_{\Lambda, \beta_{N}}(S)}\right] \quad \text { and } \\
\left|\nabla f_{N}(\omega)\right|^{2}=\sum_{(n, x) \in \mathbb{N} \times \mathbb{Z}^{2}}\left(\frac{\partial f_{N}}{\partial \omega_{n, x}}\right)^{2}=\frac{1}{\left(Z_{\Lambda, \beta_{N}}\right)^{2}} \mathrm{E}\left[\beta_{N}^{2}|S \cap \widetilde{S} \cap \Lambda| e^{H_{\Lambda, \beta_{N}}(S)+H_{\Lambda, \beta_{N}}(\widetilde{S})}\right],
\end{gathered}
$$

where $S$ and $\widetilde{S}$ are two independent copies of the random walk, and with some abuse of notation, we also denote by $S$ the random subset $\left\{\left(n, S_{n}\right)\right\}_{n \in \mathbb{N}} \subseteq \mathbb{N} \times \mathbb{Z}^{2}$.

For $\Lambda \subseteq\{1, \ldots, N\} \times \mathbb{Z}^{2}$ we have $|S \cap \widetilde{S} \cap \Lambda| \leqslant \mathcal{L}_{N}(S, \widetilde{S})$, see (3.6), where $\mathcal{L}_{N}(S, \widetilde{S})$ denotes the overlap up to time $N$ of the two trajectories $S$ and $\widetilde{S}$. On the event that $f_{N}(\omega) \geqslant a=-\log 2$, that is $Z_{\Lambda, \beta_{N}} \geqslant 1 / 2$, we can thus bound

$$
\left|\nabla f_{N}(\omega)\right|^{2} \leqslant 4 \mathrm{E}\left[\beta_{N}^{2} \mathcal{L}_{N}(S, \widetilde{S}) e^{H_{\Lambda, \beta_{N}}(S)+H_{\Lambda, \beta_{N}}(\widetilde{S})}\right]
$$

and note that, arguing as in (3.5)-(3.7), for every $\delta>0$ we have, for all $N$ large enough,

$$
\begin{aligned}
\mathbb{E} \mathrm{E}\left[\beta_{N}^{2} \mathcal{L}_{N}(S, \widetilde{S}) e^{H_{\Lambda, \beta_{N}}(S)+H_{\Lambda, \beta_{N}}(\widetilde{S})}\right] & \leqslant \mathrm{E}\left[\beta_{N}^{2} \mathcal{L}_{N}(S, \widetilde{S}) e^{(1+\delta) \beta_{N}^{2} \mathcal{L}_{N}(S, \widetilde{S})}\right] \\
& \leqslant \frac{1}{\delta} \mathrm{E}\left[e^{(1+2 \delta) \beta_{N}^{2} \mathcal{L}_{N}(S, \widetilde{S})}\right]
\end{aligned}
$$

where we used the bound $x \leqslant \frac{1}{\delta} e^{\delta x}$. Thus, for all $N$ large enough we have

$$
\mathbb{P}\left(f_{N}(\omega) \geqslant a,\left|\nabla f_{N}(\omega)\right|>c\right) \leqslant \frac{1}{c^{2}} \mathbb{E}\left[\left|\nabla f_{N}(\omega)\right|^{2} \mathbb{1}_{\left\{f_{N}(\omega) \geqslant a\right\}}\right] \leqslant \frac{4}{c^{2}} \frac{1}{\delta} \mathrm{E}\left[e^{(1+2 \delta) \beta_{N}^{2} \mathcal{L}_{N}(S, \widetilde{S})}\right] .
$$

Let us now define $\hat{\beta}^{\prime}:=\frac{1+\hat{\beta}}{2}$, so that $\hat{\beta}<\hat{\beta}^{\prime}<1$, and define $\beta_{N}^{\prime}:=\hat{\beta}^{\prime} / \sqrt{R_{N}}$, see (1.18). Then we can fix $\delta=\delta_{\hat{\beta}}>0$ small enough so that $(1+2 \delta) \beta_{N}^{2}<\lambda\left(2 \beta_{N}^{\prime}\right)-2 \lambda\left(\beta_{N}^{\prime}\right)$ (note that $\lambda(2 \beta)-2 \lambda(\beta) \sim \beta^{2}$ as $\beta \rightarrow 0$ ), hence by (3.5) and (3.2)

$$
\mathbb{P}\left(f_{N}(\omega) \geqslant a,\left|\nabla f_{N}(\omega)\right|>c\right) \leqslant \frac{4}{c^{2}} \frac{1}{\delta_{\hat{\beta}}} C_{\hat{\beta}^{\prime}} .
$$

Choosing $c=c_{\hat{\beta}}$ large enough, we can make the right hand side smaller than $\vartheta_{\hat{\beta}}$, see (3.22). Looking back at (3.20), we see that (3.19) is proved.

3.4. Proof of equation (3.4). The quantity $\mathbb{E}\left[\hat{Z}_{N, \beta_{N}}^{A}(x)^{2}\right]$ admits a representation similar to the first line of (3.1), without the constant term 1 and with the inner sum restricted to space-time points such that $\left(n_{i}, x_{i}\right) \notin A_{N}^{x}$ for some $i=1, \ldots, k$, i.e. either $n_{i}>N^{1-a_{N}}$ or $\left|x_{i}-x\right| \geqslant N^{\frac{1}{2}-\frac{a_{N}}{4}}$. Since there are $k$ space-time points, for some $j=1, \ldots, k$ we must have either $n_{j}-n_{j-1}>\frac{1}{k} N^{1-a_{N}}$ or $\left|x_{j}-x_{j-1}\right| \geqslant \frac{1}{k} N^{\frac{1}{2}-\frac{a_{N}}{4}}$ (we recall that $n_{0}=0$ 
and $x_{0}=x$ ). Defining the new variables $\ell_{i}:=n_{i}-n_{i-1}$ and $z_{i}:=x_{i}-x_{i-1}$, and enlarging the range $0<n_{1}<\ldots<n_{k} \leqslant N$ to $\ell_{1}, \ldots, \ell_{k} \in\{1, \ldots, N\}$, we can then bound

$$
\begin{aligned}
& \mathbb{E}\left[\hat{Z}_{N, \beta_{N}}^{A}(x)^{2}\right] \leqslant \sum_{k=1}^{N}\left(\sigma_{N}^{2}\right)^{k} \sum_{\substack{\ell_{1}, \ldots, \ell_{k} \in\{1, \ldots, N\} \\
z_{1}, \ldots, z_{k} \in \mathbb{Z}^{2}}} \sum_{j=1}^{k}\left(\mathbb{1}_{\left\{\ell_{j}>\frac{1}{k} N^{1-a_{N}}\right\}}\right. \\
& \left.+\mathbb{1}_{\left\{\ell_{j} \leqslant \frac{1}{k} N^{1-a_{N}},\left|z_{j}\right| \geqslant \frac{1}{k} N^{\frac{1}{2}-\frac{a_{N}}{4}}\right\}}\right) \prod_{i=1}^{k} q_{\ell_{i}}\left(z_{i}\right)^{2} .
\end{aligned}
$$

We now switch the sum over $j$ with the double sum over $\ell_{i}, z_{i}$ 's. We can sum over all variables $z_{j}$ 's with $i \neq j$, replacing each kernel $q_{\ell_{i}}\left(z_{i}\right)^{2}$ by $q_{2 \ell_{i}}(0)$ (see (1.17)), and then sum $q_{2 \ell_{i}}(0)$ for all $\ell_{i}$ 's with $i \neq j$, which gives $\left(R_{N}\right)^{k-1}$ (see again (1.17)). This yields

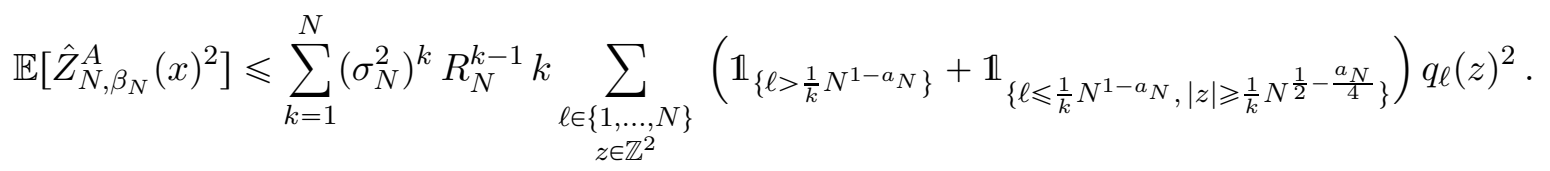

We now consider separately the contributions of the two indicator functions.

- Recalling (1.17), (1.18), (2.15), the contribution of $\left\{\ell>\frac{1}{k} N^{1-a_{N}}\right\}$ is controlled by

$$
\begin{array}{r}
\sum_{k=1}^{N}\left(\sigma_{N}^{2}\right)^{k} R_{N}^{k-1} k \sum_{\frac{1}{k} N^{1-a_{N}<\ell \leqslant N}} q_{2 \ell}(0) \leqslant C \sum_{k=1}^{N} k\left(\hat{\beta}^{2}\right)^{k} \frac{1}{\log N} \sum_{\frac{1}{k} N^{1-a_{N}<\ell \leqslant N}} \frac{1}{\ell} \\
\leqslant C^{\prime} \sum_{k=1}^{N} k\left(\hat{\beta}^{2}\right)^{k} \frac{a_{N} \log N+\log k}{\log N} \leqslant C^{\prime}\left(\widetilde{C}_{\hat{\beta}} a_{N}+\hat{C}_{\hat{\beta}} \frac{1}{\log N}\right),
\end{array}
$$

where $\widetilde{C}_{\hat{\beta}}:=\sum_{k=1}^{\infty} k\left(\hat{\beta}^{2}\right)^{k}$ and $\hat{C}_{\hat{\beta}}:=\sum_{k=1}^{\infty} k(\log k)\left(\hat{\beta}^{2}\right)^{k}$ are finite, $\hat{\beta}$-dependent constants. This contribution is consistent with (3.4) (recall (2.2) ).

- The contribution of $\left\{\ell \leqslant \frac{1}{k} N^{1-a_{N}},|z| \geqslant \frac{1}{k} N^{\frac{1}{2}-\frac{a_{N}}{4}}\right\}$ is given by

$$
\sum_{k=1}^{N}\left(\sigma_{N}^{2}\right)^{k} R_{N}^{k-1} k \sum_{1 \leqslant \ell \leqslant \frac{1}{k} N^{1-a_{N}}} \sum_{|z|>\frac{1}{k} N^{\frac{1}{2}-\frac{a_{N}}{4}}} q_{\ell}(z)^{2} .
$$

Note that we can enlarge the range of the last sum to $|z|>\vartheta \sqrt{\ell}$, with $\vartheta=N^{\frac{a_{N}}{4}} / \sqrt{k}$. Note that $\sup _{z \in \mathbb{Z}^{2}} q_{\ell}(z) \leqslant c / \ell$, by Gnedenko's local limit theorem. Then, by Gaussian estimates for the simple random walk on $\mathbb{Z}^{2}$, there is $\eta>0$ such that

$$
\sum_{|z|>\vartheta \sqrt{\ell}} q_{\ell}(z)^{2} \leqslant \frac{c}{\ell} \mathrm{P}\left(\left|S_{\ell}\right|>\vartheta \sqrt{\ell}\right) \leqslant \frac{c}{\ell} e^{-\eta \vartheta^{2}}, \quad \forall \ell \in \mathbb{N}, \forall \vartheta>0 .
$$

Then we can bound (3.24) by a constant multiple of

$$
\sum_{k=1}^{N}\left(\hat{\beta}^{2}\right)^{k} k \frac{1}{R_{N}} \sum_{1 \leqslant \ell \leqslant N} \frac{c}{\ell} e^{-\eta \vartheta^{2}} \leqslant C \sum_{k=1}^{N}\left(\hat{\beta}^{2}\right)^{k} k e^{-\eta \frac{a_{N} \frac{a^{2}}{k}}{k}} .
$$

We split the sum according to $k \leqslant\left(N^{\frac{a_{N}}{2}}\right)^{1 / 2}$ and $k>\left(N^{\frac{a_{N}}{2}}\right)^{1 / 2}$, getting the bound

$$
\left\{\sum_{k=1}^{\infty}\left(\hat{\beta}^{2}\right)^{k} k\right\} e^{-\eta\left(N^{\frac{a}{2}}\right)^{1 / 2}}+\left\{\sum_{k=1}^{\infty} \hat{\beta}^{k} k\right\} \hat{\beta}^{\left(N^{\frac{a}{2}}\right)^{1 / 2}} .
$$


Both brackets are finite, $\hat{\beta}$-dependent constants, while the other factors are both $o\left(a_{N}\right)$ as $N \rightarrow \infty$, by (2.2), because $\hat{\beta}<1$ and $N^{\frac{a_{N}}{2}}=\exp \left(\frac{1}{2}(\log N)^{\gamma}\right) \gg \log N$.

This completes the proof of (3.4).

\section{EDWARDS-WILKINSON FLUCTUATIONS FOR DIRECTED POLYMER}

In this section, we prove Theorem 1.6, which consists of proving Propositions 2.1, 2.2, 2.3. and 2.4 as described in Section 2. The proofs are given in the following subsections.

4.1. Proof of Proposition 2.1. Recalling (2.8), we need to show that

$$
\frac{\log N}{N^{2}} \sum_{x, y \in \mathbb{Z}^{2}} \operatorname{Cov}\left[O_{N}(x), O_{N}(y)\right] \phi\left(\frac{x}{\sqrt{N}}\right) \phi\left(\frac{y}{\sqrt{N}}\right) \underset{N \rightarrow \infty}{\longrightarrow} 0 .
$$

By translation invariance and Cauchy-Schwarz, it suffices to show that for any $x \in \mathbb{Z}^{2}$,

$$
(\log N) \mathbb{E}\left[O_{N}(x)^{2}\right] \underset{N \rightarrow \infty}{\longrightarrow} 0 .
$$

We recall that $O_{N}(x)$ is defined in (2.7), and in view of (2.5) we can write

$$
O_{N}(x)=\log \left(1+\frac{\hat{Z}_{N, \beta_{N}}^{A}(x)}{Z_{N, \beta_{N}}^{A}(x)}\right)-\frac{\hat{Z}_{N, \beta_{N}}^{A}(x)}{Z_{N, \beta_{N}}^{A}(x)}
$$

We can bound, for a suitable constant $C<\infty$,

$$
|\log (1+y)-y| \leqslant C \cdot\left\{\begin{array}{ll}
\sqrt{\frac{|y|}{1+y}} & \text { if }-1<y<0 \\
y^{2} & \text { if }-\frac{1}{2} \leqslant y \leqslant \frac{1}{2} \\
|y| & \text { if } 0<y<\infty
\end{array} .\right.
$$

The three domains are chosen to overlap on purpose: in fact, we will apply these inequalities in the domains $\left(-1,-a_{N}^{2 / 7}\right),\left[-a_{N}^{2 / 7}, a_{N}^{2 / 7}\right]$ and $\left(a_{N}^{2 / 7}, \infty\right)$ (recall $a_{N}$ from (2.2)). We define

$$
D_{N}^{ \pm}:=\left\{ \pm \frac{\hat{Z}_{N, \beta_{N}}^{A}(x)}{Z_{N, \beta_{N}}^{A}(x)}>a_{N}^{2 / 7}\right\}, \quad D_{N}:=D_{N}^{+} \cup D_{N}^{-}=\left\{\left|\frac{\hat{Z}_{N, \beta_{N}}^{A}(x)}{Z_{N, \beta_{N}}^{A}(x)}\right|>a_{N}^{2 / 7}\right\},
$$

and we bound

$$
\begin{aligned}
\mathbb{P}\left(D_{N}\right) & \leqslant \mathbb{P}\left(Z_{N, \beta_{N}}^{A}(x)<a_{N}^{1 / 7}\right)+\mathbb{P}\left(\left|\hat{Z}_{N, \beta_{N}}^{A}(x)\right|>a_{N}^{3 / 7}\right) \\
& \leqslant a_{N}^{1 / 7} \mathbb{E}\left[Z_{N, \beta_{N}}^{A}(x)^{-1}\right]+a_{N}^{-6 / 7} \mathbb{E}\left[\hat{Z}_{N, \beta_{N}}^{A}(x)^{2}\right] \leqslant\left(C_{2, \hat{\beta}}+C_{\hat{\beta}}\right) a_{N}^{1 / 7},
\end{aligned}
$$

thanks to (3.15) and (3.4). Then by (4.2)

$$
\frac{1}{C} \mathbb{E}\left[O_{N}(x)^{2}\right] \leqslant \mathbb{E}\left[\left(\frac{\hat{Z}_{N, \beta_{N}}^{A}(x)}{Z_{N, \beta_{N}}^{A}(x)}\right)^{4} \mathbb{1}_{D_{N}^{c}}\right]+\mathbb{E}\left[\left(\frac{\hat{Z}_{N, \beta_{N}}^{A}(x)}{Z_{N, \beta_{N}}^{A}(x)}\right)^{2} \mathbb{1}_{D_{N}^{+}}\right]+\mathbb{E}\left[\frac{\left|\hat{Z}_{N, \beta_{N}}^{A}(x) / Z_{N, \beta_{N}}^{A}(x)\right|}{1+\hat{Z}_{N, \beta_{N}}^{A}(x) / Z_{N, \beta_{N}}^{A}(x)} \mathbb{1}_{D_{N}^{-}}\right],
$$

and given that

$$
1+\frac{\hat{Z}_{N, \beta_{N}}^{A}(x)}{Z_{N, \beta_{N}}^{A}(x)}=\frac{Z_{N, \beta_{N}}(x)}{Z_{N, \beta_{N}}^{A}(x)}
$$


we can choose $p=p_{\hat{\beta}}>2$ close to 2 as in (3.12) such that

$$
\begin{aligned}
\frac{1}{C} \mathbb{E}\left[O_{N}(x)^{2}\right] & \leqslant \mathbb{E}\left[\left(\frac{\hat{Z}_{N, \beta_{N}}^{A}(x)}{Z_{N, \beta_{N}}^{A}(x)}\right)^{4} \mathbb{1}_{D_{N}^{c}}\right]+\mathbb{E}\left[\left(\frac{\hat{Z}_{N, \beta_{N}}^{A}(x)}{Z_{N, \beta_{N}}^{A}(x)}\right)^{2} \mathbb{1}_{D_{N}^{+}}\right]+\mathbb{E}\left[\left|\frac{\hat{Z}_{N, \beta_{N}}^{A}(x)}{Z_{N, \beta_{N}}(x)}\right| \mathbb{1}_{D_{N}^{-}}\right] \\
& \leqslant a_{N}^{\frac{8}{7}}+\mathbb{E}\left[\hat{Z}_{N, \beta_{N}}^{A}(x)^{p}\right]^{\frac{2}{p}}\left(\mathbb{E}\left[Z_{N, \beta_{N}}^{A}(x)^{-\frac{2 p}{p-2}} \mathbb{1}_{D_{N}^{+}}\right]^{1-\frac{2}{p}}+\mathbb{E}\left[Z_{N, \beta_{N}}(x)^{-2} \mathbb{1}_{D_{N}^{-}}\right]^{\frac{1}{2}}\right) \\
& \leqslant a_{N}^{\frac{8}{7}}+C_{\hat{\beta}}^{\prime} a_{N}\left(\mathbb{E}\left[Z_{N, \beta_{N}}^{A}(x)^{-\frac{4 p}{p-2}}\right]^{\frac{1}{2}-\frac{1}{p}} \mathbb{P}\left(D_{N}\right)^{\frac{1}{2}-\frac{1}{p}}+\mathbb{E}\left[Z_{N, \beta_{N}}(x)^{-4}\right]^{\frac{1}{4}} \mathbb{P}\left(D_{N}\right)^{\frac{1}{4}}\right) \\
& \leqslant a_{N}^{\frac{8}{7}}+C_{\hat{\beta}}^{\prime} a_{N} \mathbb{P}\left(D_{N}\right)^{\frac{1}{4} \wedge\left(\frac{1}{2}-\frac{1}{p}\right)} \leqslant C_{\hat{\beta}}^{\prime} a_{N}^{1+\frac{1}{7}\left(\frac{1}{2}-\frac{1}{p}\right)}
\end{aligned}
$$

where the second last inequality holds by (3.12), (3.14) and (3.15), in the last inequality we applied (4.3), and $C_{\hat{\beta}}^{\prime}<\infty$ is a generic constant depending only on $\hat{\beta}$. Recall from (2.2) that $a_{N}=(\log N)^{\gamma-1}$. We can then choose $\gamma \in\left(0, \gamma^{*}\right)$ with $\gamma^{*}>0$ small enough such that

$$
\mathbb{E}\left[O_{N}(x)^{2}\right] \leqslant C_{\hat{\beta}}^{\prime} a_{N}^{1+\frac{1}{7}\left(\frac{1}{2}-\frac{1}{p}\right)}=C_{\hat{\beta}}^{\prime}(\log N)^{-(1-\gamma)\left(1+\frac{1}{7}\left(\frac{1}{2}-\frac{1}{p}\right)\right)}=o\left((\log N)^{-1}\right) .
$$

Therefore (4.1) holds.

4.2. Proof of Proposition 2.2, We need to show that

$$
\frac{\log N}{N^{2}} \sum_{x, y \in \mathbb{Z}^{2}} \mathbb{C o v}\left[\log Z_{N, \beta_{N}}^{A}(x), \log Z_{N, \beta_{N}}^{A}(y)\right] \phi\left(\frac{x}{\sqrt{N}}\right) \phi\left(\frac{y}{\sqrt{N}}\right) \underset{N \rightarrow \infty}{\longrightarrow} 0 .
$$

We recall that $Z_{N, \beta_{N}}^{A}(x)$ depends only on the disorder within set $A_{N}^{x}$, defined in (2.3), hence $Z_{N, \beta_{N}}^{A}(x)$ and $Z_{N, \beta_{N}}^{A}(y)$ are independent for $|x-y|>2 N^{\frac{1}{2}-\frac{a_{N}}{4}}$. By Cauchy-Schwarz and (3.16), we can bound the left hand side of (4.5) as follows:

$$
\begin{aligned}
& C_{2, \hat{\beta}} \frac{\log N}{N^{2}} \sum_{x, y \in \mathbb{Z}^{2}:|y-x| \leqslant 2 N^{\frac{1}{2}-\frac{a_{N}}{4}}} \phi\left(\frac{x}{\sqrt{N}}\right) \phi\left(\frac{y}{\sqrt{N}}\right) \leqslant c C_{2, \hat{\beta}} \frac{\log N}{N^{2}} N^{1-\frac{a_{N}}{2}}|\phi|_{\infty} \sum_{x \in \mathbb{Z}^{2}}\left|\phi\left(\frac{x}{\sqrt{N}}\right)\right| \\
& \leqslant c^{\prime} C_{2, \hat{\beta}}(\log N) N^{-\frac{a_{N}}{2}}|\phi|_{\infty}|\phi|_{L^{1}\left(\mathbb{R}^{2}\right)}=c^{\prime} C_{2, \hat{\beta}} e^{\log (\log N)-\frac{1}{2}(\log N)^{\gamma}}|\phi|_{\infty}|\phi|_{L^{1}\left(\mathbb{R}^{2}\right)} \underset{N \rightarrow \infty}{\longrightarrow} 0,
\end{aligned}
$$

where $c, c^{\prime}$ are generic constants, and the last equality holds by definition of $a_{N}$ in (2.2) .

4.3. Proof of Proposition 2.3. We need to show that

$$
\frac{\sqrt{\log N}}{N} \sum_{x \in \mathbb{Z}^{2}} \frac{\hat{Z}_{N, \beta_{N}}^{A}(x)}{Z_{N, \beta_{N}}^{A}(x)} \phi\left(\frac{x}{\sqrt{N}}\right)-\frac{\sqrt{\log N}}{N} \sum_{x \in \mathbb{Z}^{2}}\left(Z_{N, \beta_{N}}^{B^{\geqslant}}(x)-1\right) \phi\left(\frac{x}{\sqrt{N}}\right) \underset{N \rightarrow \infty}{\stackrel{L^{1}(\mathbb{P})}{\longrightarrow}} 0 .
$$

We recall that $B_{N}^{\geqslant}:=\left(\left(N^{1-9 a_{N} / 40}, N\right] \cap \mathbb{N}\right) \times \mathbb{Z}^{2}$, see (2.10). We define new subsets

$$
\begin{gathered}
B_{N}:=\left(\left(N^{1-a_{N}}, N\right] \cap \mathbb{N}\right) \times \mathbb{Z}^{2}, \\
C_{N}^{x}:=\left\{(n, z) \in \mathbb{N} \times \mathbb{Z}^{2}: n \leqslant N^{1-a_{N}},|z-x| \geqslant N^{\frac{1}{2}-\frac{a_{N}}{4}}\right\},
\end{gathered}
$$

and we introduce new "partition functions":

$$
\begin{aligned}
& Z_{N, \beta_{N}}^{A, C}(x):=\sum_{\tau \subset\{1, \ldots, N\} \times \mathbb{Z}^{2}: \tau \cap C_{N}^{x} \neq \varnothing} \sigma_{N}^{|\tau|} \boldsymbol{q}^{(0, x)}(\tau) \boldsymbol{\xi}(\tau), \\
& Z_{N, \beta_{N}}^{A, B}(x):=\sum_{\tau \subset A_{N}^{x} \cup B_{N}: \tau \cap B_{N} \neq \varnothing} \sigma_{N}^{|\tau|} \boldsymbol{q}^{(0, x)}(\tau) \boldsymbol{\xi}(\tau),
\end{aligned}
$$




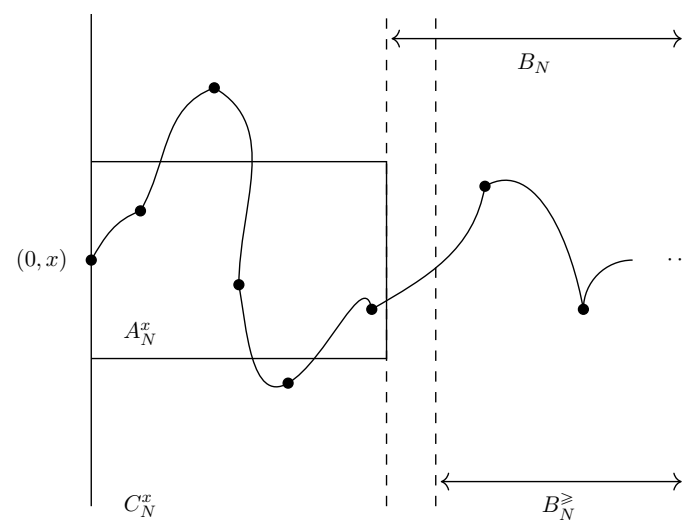

(A) Partition function $Z_{N, \beta_{N}}^{A, C}(x)$.

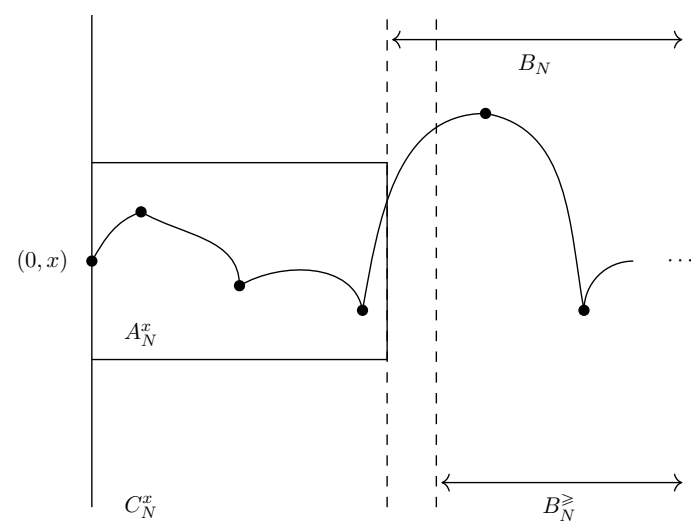

(B) Partition function $Z_{N, \beta_{N}}^{A, B}$.

Figure 2. The figures depict the chaos expansions of $Z_{N, \beta_{N}}^{A, C}(x), Z_{N, \beta_{N}}^{A, B}(x)$. Each term in the expansion for $Z_{N, \beta_{N}}^{A, C}(x)$ must include disorder from $C_{N}^{x}$; while each term in the expansion for $Z_{N, \beta_{N}}^{A, B}(x)$ contain only disorder from $A_{N}^{x} \cup B_{N}$, with at least some disorder from $B_{N}$.

similar to the polynomial chaos expansions for $Z_{N, \beta_{N}}, Z_{N, \beta_{N}}^{A}$ and $Z_{N, \beta_{N}}^{B \geqslant}$ in (2.18)-(2.19). See Figure 2 for a graphical representation of the chaos expansions.

Recall that $A_{N}^{x}$ was defined in (2.3), and note that $\left(\{1, \ldots, N\} \times \mathbb{Z}^{2}\right) \backslash A_{N}^{x}=C_{N}^{x} \cup B_{N}$. We can then decompose $\hat{Z}_{N, \beta_{N}}^{A}(x)$, defined in (2.5), as follows:

$$
\hat{Z}_{N, \beta_{N}}^{A}(x)=Z_{N, \beta_{N}}^{A, B}(x)+Z_{N, \beta_{N}}^{A, C}(x) .
$$

We split the sequel in three steps. The first step is:

(1) We will show that the contribution of the term $Z_{N, \beta_{N}}^{A, C}$ is negligible for (4.6).

To treat the term $Z_{N, \beta_{N}}^{A, B}(x)$, we decompose its chaos expansion (4.10) according to the last point $(t, w)$ of $\tau$ that lies in $A_{N}^{x}$ and the first point $(r, z)$ of $\tau$ that lies in $B_{N}$ :

$$
Z_{N, \beta_{N}}^{A, B}(x)=\sum_{\substack{(t, w) \in\{(0, x)\} \cup A_{N}^{x} \\(r, z) \in B_{N}}} Z_{0, t, \beta_{N}}^{A}(x, w) \cdot q_{r-t}(z-w) \cdot \sigma_{N} \xi_{r, z} \cdot Z_{r, N, \beta_{N}}(z),
$$

where $Z_{0, t, \beta_{N}}^{A}(x, w)$ is the "point-to-point" partition function from $(0, x)$ to $(t, w)$, defined by $Z_{0, t, \beta_{N}}^{A}(x, w):=1$ if $(t, w)=(0, x)$ and by

$$
Z_{0, t, \beta_{N}}^{A}(x, w):=\sum_{\tau \subset A_{N}^{x} \cap\left([0, t] \times \mathbb{Z}^{2}\right): \tau \ni(t, w)} \sigma_{N}^{|\tau|} \boldsymbol{q}^{(0, x)}(\tau) \boldsymbol{\xi}(\tau) \quad \text { if } t>0,
$$

while $Z_{r, N, \beta_{N}}(z)$ is the "point-to-plane" partition function starting at $(r, z)$ and running until time $N$ :

$$
Z_{r, N, \beta_{N}}(z):=\sum_{\tau \subset\{r+1, \ldots, N\} \times \mathbb{Z}^{2}} \sigma_{N}^{|\tau|} \boldsymbol{q}^{(r, z)}(\tau) \boldsymbol{\xi}(\tau) .
$$

The next steps are:

(2) We will show that in (4.12) the contribution from $r<N^{1-9 a_{N} / 40}$ is negligible for (4.6). 
(3) We will show that in (4.12) we can replace the kernel $q_{r-t}(z-w)$ by $q_{r}(z-x)$, i.e. the transition kernel from $(0, x)$ to $(r, z)$, because their difference is negligible for (4.6).

Finally, note that when we restrict the sum in (4.12) to $r \geqslant N^{1-9 a_{N} / 40}$, i.e. to $(r, z) \in B_{N}^{\geqslant}$ (recall (2.10) $)$, and we replace $q_{r-t}(z-w)$ by $q_{r}(z-x)$, the right hand side of (4.12) becomes exactly $Z_{N, \beta_{N}}^{A}(x)\left(Z_{N, \beta_{N}}^{B \geqslant}(x)-1\right)$ (recall (2.19) $)$. This completes the proof of (4.6).

It remains to prove the three steps stated above.

Step (11). We show that the contribution of $Z_{N, \beta_{N}}^{A, C}$ in (4.11) to (4.6) is negligible, that is,

$$
\frac{\sqrt{\log N}}{N} \sum_{x \in \mathbb{Z}^{2}} \frac{Z_{N, \beta_{N}}^{A, C}(x)}{Z_{N, \beta_{N}}^{A}(x)} \phi\left(\frac{x}{\sqrt{N}}\right) \underset{N \rightarrow \infty}{\stackrel{L^{2}(\mathbb{P})}{\longrightarrow}} 0 .
$$

Since the chaos expansion of $Z_{N, \beta_{N}}^{A, C}(x)$ in (4.9) contains disorder $\xi$ outside $A_{N}^{x}$, not contained in the expansion of $Z_{N, \beta_{N}}^{A}(x)$, we have that $\mathbb{E}\left[Z_{N, \beta_{N}}^{A, C}(x) / Z_{N, \beta_{N}}^{A}(x)\right]=0$ thus $L^{2}(\mathbb{P})$ and variance computations are equivalent. We then have

$\operatorname{Var}\left(\frac{\sqrt{\log N}}{N} \sum_{x \in \mathbb{Z}^{2}} \frac{Z_{N, \beta_{N}}^{A, C}(x)}{Z_{N, \beta_{N}}^{A}(x)} \phi\left(\frac{x}{\sqrt{N}}\right)\right)=\frac{\log N}{N^{2}} \sum_{x, y \in \mathbb{Z}^{2}} \mathbb{E}\left[\frac{Z_{N, \beta_{N}}^{A, C}(x)}{Z_{N, \beta_{N}}^{A}(x)} \cdot \frac{Z_{N, \beta_{N}}^{A, C}(y)}{Z_{N, \beta_{N}}^{A}(y)}\right] \phi\left(\frac{x}{\sqrt{N}}\right) \phi\left(\frac{y}{\sqrt{N}}\right)$.

By Cauchy-Schwarz, we can further bound this as follows, for some constant $c$ :

$$
\begin{gathered}
\frac{\log N}{N^{2}} \mathbb{E}\left[\left(\frac{Z_{N, \beta_{N}}^{A, C}(0)}{Z_{N, \beta_{N}}^{A}(0)}\right)^{2}\right] \sum_{x, y \in \mathbb{Z}^{2}}\left|\phi\left(\frac{x}{\sqrt{N}}\right)\right|\left|\phi\left(\frac{y}{\sqrt{N}}\right)\right| \leqslant c \log N \cdot|\phi|_{L^{1}\left(\mathbb{R}^{2}\right)}^{2} \mathbb{E}\left[\left(\frac{Z_{N, \beta_{N}}^{A, C}(0)}{Z_{N, \beta_{N}}^{A}(0)}\right)^{2}\right] \\
\leqslant c \log N \cdot|\phi|_{L^{1}\left(\mathbb{R}^{2}\right)}^{2} \mathbb{E}\left[Z_{N, \beta_{N}}^{A, C}(0)^{2 p}\right]^{1 / p} \mathbb{E}\left[\frac{1}{Z_{N, \beta_{N}}^{A}(0)^{2 q}}\right]^{1 / q}
\end{gathered}
$$

where in the last step we used Hölder inequality with parameters $(p, q)$ with $p^{-1}+q^{-1}=1$, and $p$ will be chosen sufficiently close to 1 , to be determined below. The term $\mathbb{E}\left[Z_{N, \beta_{N}}^{A}(0)^{-2 q}\right]^{1 / q}$ can be uniformly bounded by the negative moment estimate (3.15).

We can use hypercontractivity, see (3.10), to bound

$$
\mathbb{E}\left[Z_{N, \beta_{N}}^{A, C}(0)^{2 p}\right]^{1 / p} \leqslant \sum_{\tau \subset\{1, \ldots, N\} \times \mathbb{Z}^{2}: \tau \cap C_{N}^{0} \neq \varnothing}\left(c_{2 p} \sigma_{N}\right)^{2|\tau|} \boldsymbol{q}^{(0)}(\tau)^{2} .
$$

The right hand side is the second moment of the partition function, see (3.1), except that $\sigma_{N}$ is replaced by $c_{2 p} \sigma_{N}$ (which corresponds asymptotically to replacing $\hat{\beta}$ by $\hat{\beta}^{\prime}:=c_{2 p} \hat{\beta}$, see (2.15) and (1.18)) and the random walk $S$ must satisfy $\max \left\{\left|S_{n}\right|: n<N^{1-a_{N}}\right\}>N^{1 / 2-a_{N} / 4}$. In particular, recalling (3.7) and (3.6), this can be bounded by

$$
\mathrm{E}\left[e^{(1+o(1))\left(c_{2 p} \beta_{N}\right)^{2} \mathcal{L}_{N^{1-a_{N}}}\left(S^{(1)}, S^{(2)}\right)} ; \max _{n \leqslant N^{1-a_{N}}}\left|S_{n}^{(i)}\right|>N^{\frac{1}{2}-\frac{a_{N}}{4}}, \text { for } i=1,2\right]
$$

where $S^{(1)}, S^{(2)}$ are two independent random walk copies. This is bounded via Hölder by

$$
\mathrm{E}\left[e^{(1+o(1)) p\left(c_{2 p} \beta_{N}\right)^{2} \mathcal{L}_{N^{1-a_{N}}}\left(S^{(1)}, S^{(2)}\right)}\right]^{1 / p} \mathrm{P}\left(\max _{n \leqslant N^{1-a_{N}}}\left|S_{n}\right|>N^{\frac{1}{2}-\frac{a_{N}}{4}}\right)^{2 / q} .
$$

We can now choose $p>1$ sufficiently close to 1 so that $\sqrt{p} c_{2 p} \hat{\beta}<1$, i.e. still subcritical, which is possible because $\lim _{p \rightarrow 1} c_{2 p}=1$, see (3.11). Hence the expectation above is 
uniformly bounded in $N$ as shown in Section 3.1. On the other hand, standard moderate deviation estimates for the simple symmetric random walk show that

$$
\mathrm{P}\left(\max _{n \leqslant N^{1-a_{N}}}\left|S_{n}\right|>N^{\frac{1}{2}-\frac{a_{N}}{4}}\right) \leqslant \exp \left(-c N^{a_{N} / 2}\right)=\exp \left(-c e^{(\log N)^{\gamma} / 2}\right),
$$

where we recall that $a_{N}=(\log N)^{\gamma-1}$, see (2.2). Inserting these estimates in (4.16), we get

$$
\operatorname{Var}\left(\frac{\sqrt{\log N}}{N} \sum_{x \in \mathbb{Z}^{2}} \frac{Z_{N, \beta_{N}}^{A, C}(x)}{Z_{N, \beta_{N}}^{A}(x)} \phi\left(\frac{x}{\sqrt{N}}\right)\right) \leqslant c \log N \cdot|\phi|_{L^{1}\left(\mathbb{R}^{2}\right)}^{2} \exp \left(-c e^{(\log N)^{\gamma} / 2}\right) \underset{N \rightarrow \infty}{\longrightarrow} 0 .
$$

Step (2). We show that in the chaos expansion (4.12) for $Z_{N, \beta_{N}}^{A, B}(x)$, the contribution from $(r, z)$ with $r<N^{1-9 a_{N} / 40}$ is negligible for (4.6). The contribution we are after is

$$
Z_{N, \beta_{N}}^{A, B^{<}}(x):=\sum_{\substack{(t, w) \in A_{N}^{x} \\(r, z) \in B_{N}: r<N^{1-9 a_{N} / 40}}} Z_{0, t, \beta_{N}}^{A}(x, w) \cdot q_{r-t}(z-w) \cdot \sigma_{N} \xi_{r, z} \cdot Z_{r, N, \beta_{N}}(z),
$$

and we want to show that

$$
\mathbb{E}\left[\left(\frac{\sqrt{\log N}}{N} \sum_{x \in \mathbb{Z}^{2}} \phi\left(\frac{x}{\sqrt{N}}\right) \frac{Z_{N, \beta_{N}}^{A, B^{<}}(x)}{Z_{N, \beta_{N}}^{A}(x)}\right)^{2}\right] \underset{N \rightarrow \infty}{\longrightarrow} 0 .
$$

The left hand side of (4.19) equals

$$
\frac{\log N}{N^{2}} \sum_{x, y \in \mathbb{Z}^{2}} \phi\left(\frac{x}{\sqrt{N}}\right) \phi\left(\frac{y}{\sqrt{N}}\right) \mathbb{E}\left[\frac{Z_{N, \beta_{N}}^{A, B^{<}}(x)}{Z_{N, \beta_{N}}^{A}(x)} \cdot \frac{Z_{N, \beta_{N}}^{A, B^{<}}(y)}{Z_{N, \beta_{N}}^{A}(y)}\right] .
$$

We can restrict the summation over $x, y$ to $|x-y|>N^{\frac{1}{2}-\frac{a_{N}}{10}}$. Indeed, in the complementary regime, we first bound the expectation in (4.20) by Cauchy-Schwarz and obtain the bound

$$
\begin{aligned}
\frac{\log N}{N^{2}} \mathbb{E}\left[\left(\frac{Z_{N, \beta_{N}}^{A, B^{<}}(0)}{Z_{N, \beta_{N}}^{A}(0)}\right)^{2}\right] \sum_{|x-y| \leqslant N}{\frac{1}{2}-\frac{a_{N}}{10}}_{1}\left|\phi\left(\frac{x}{\sqrt{N}}\right)\right|\left|\phi\left(\frac{y}{\sqrt{N}}\right)\right| \\
\leqslant N^{1-\frac{a_{N}}{5}} \frac{\log N}{N} \mathbb{E}\left[\left(\frac{Z_{N, \beta_{N}}^{A, B^{<}}(0)}{Z_{N, \beta_{N}}^{A}(0)}\right)^{2}\right]|\phi|_{\infty}|\phi|_{L^{1}\left(\mathbb{R}^{2}\right)} \\
=(\log N) e^{-\frac{1}{5}(\log N)^{\gamma}} \mathbb{E}\left[\left(\frac{Z_{N, \beta_{N}}^{A, B^{<}}(0)}{Z_{N, \beta_{N}}^{A}(0)}\right)^{2}\right]|\phi|_{\infty}|\phi|_{L^{1}\left(\mathbb{R}^{2}\right)}
\end{aligned}
$$

which goes to zero as $N \rightarrow \infty$, since expectation can be bounded via Hölder with an exponent $p$ for $Z_{N, \beta_{N}}^{A, B^{<}}(0)^{2}$ chosen sufficiently close to one, so that the hypecontractivity bound (3.12) can be applied, while the negative moment $\mathbb{E}\left[Z_{N, \beta_{N}}^{A}(0)^{-2 q}\right]$ can be bounded by (3.15). The argument is the same as that for (4.16) and we omit the details. 
To deal with (4.20) when $(x, y) \in I^{>}:=\left\{x, y \in \mathbb{Z}^{2}:|x-y|>N^{\frac{1}{2}-\frac{a_{N}}{10}}\right\}$, we use the chaos expansion for $Z_{N, \beta_{N}}^{A, B^{<}}$, (4.18), and write (4.20) in this case as follows (recall that $\mathbb{E}\left[\xi^{2}\right]=1$ ):

$$
\begin{aligned}
\frac{\sigma_{N}^{2} \log N}{N^{2}} \sum_{x, y \in I^{>}} \phi\left(\frac{x}{\sqrt{N}}\right) \phi\left(\frac{y}{\sqrt{N}}\right) \sum_{\substack{(t, w) \in A_{N}^{x} \\
(s, v) \in A_{N}^{y}}} \mathbb{E}\left[\frac{Z_{0, t, \beta_{N}}^{A}(x, w)}{Z_{N, \beta_{N}}^{A}(x)}\right] \mathbb{E}\left[\frac{Z_{0, s, \beta_{N}}^{A}(y, v)}{Z_{N, \beta_{N}}^{A}(y)}\right] \\
\times \sum_{(r, z) \in B_{N}: r<N^{1-9 a_{N} / 40}} q_{r-t}(z-w) q_{r-s}(z-v) \mathbb{E}\left[Z_{r, N, \beta_{N}}(z)^{2}\right]
\end{aligned}
$$

where the first point $(r, z) \in B_{N}$ in the expansion for $Z_{N, \beta_{N}}^{A}(x)$ and $Z_{N, \beta_{N}}^{A}(y)$ must match because an unmatched $(r, z)$ gives $\mathbb{E}\left[\xi_{r, z}\right]=0$, and we used the independence between

$$
\frac{Z_{0, t, \beta_{N}}^{A}(x, w)}{Z_{N, \beta_{N}}^{A}(x)}, \quad \frac{Z_{0, s, \beta_{N}}^{A}(y, v)}{Z_{N, \beta_{N}}^{A}(y)}, \quad \text { and } \quad Z_{r, N, \beta_{N}}(z),
$$

because they depend on disorder in the disjoint regions $A_{N}^{x}, A_{N}^{y}$ and $B_{N}$.

We can simplify (4.21) by noticing that $\mathbb{E}\left[Z_{r, N, \beta_{N}}(z)^{2}\right]$ is independent of $z$ and that $\sum_{z} q_{r-t}(z-w) q_{r-s}(z-v)=q_{2 r-t-s}(w-v)$. Thus we can write it as

$$
\begin{gathered}
\frac{\sigma_{N}^{2} \log N}{N^{2}} \sum_{x, y \in I^{>}} \phi\left(\frac{x}{\sqrt{N}}\right) \phi\left(\frac{y}{\sqrt{N}}\right) \sum_{\substack{(t, w) \in A_{N}^{x} \\
(s, v) \in A_{N}^{y}}} \mathbb{E}\left[\frac{Z_{0, t, \beta_{N}}^{A}(x, w)}{Z_{N, \beta_{N}}^{A}(x)}\right] \mathbb{E}\left[\frac{Z_{0, s, \beta_{N}}^{A}(y, v)}{Z_{N, \beta_{N}}^{A}(y)}\right] \\
\times \sum_{N^{1-a_{N}}<r<N^{1-9 a_{N} / 40}} q_{2 r-t-s}(w-v) \mathbb{E}\left[Z_{r, N, \beta_{N}}(0)^{2}\right]
\end{gathered}
$$

Note that $\mathbb{E}\left[Z_{r, N, \beta_{N}}(0)^{2}\right] \leqslant \mathbb{E}\left[Z_{N, \beta_{N}}(0)^{2}\right] \leqslant C_{\hat{\beta}}$ uniformly in $N$ by (3.2). Moreover,

$$
\left|\mathbb{E}\left[\frac{Z_{0, t, \beta_{N}}^{A}(x, w)}{Z_{N, \beta_{N}}^{A}(x)}\right]\right| \leqslant \mathbb{E}\left[Z_{0, t, \beta_{N}}^{A}(x, w)^{2}\right]^{1 / 2} \mathbb{E}\left[\frac{1}{Z_{N, \beta_{N}}^{A}(x)^{2}}\right]^{1 / 2} \leqslant C_{2, \hat{\beta}} \mathbb{E}\left[Z_{0, t, \beta_{N}}^{A}(x, w)^{2}\right]^{1 / 2}
$$

where the constant $C_{2, \hat{\beta}}$ comes from the negative moment bound (3.15). The same bound holds with $(x, t, w)$ replaced by $(y, s, v)$. Therefore (4.22) can be bounded by

$$
\begin{aligned}
& C_{\hat{\beta}} C_{2, \hat{\beta}} \frac{\sigma_{N}^{2} \log N}{N^{2}} \sum_{x, y \in I^{>}}\left|\phi\left(\frac{x}{\sqrt{N}}\right)\right|\left|\phi\left(\frac{y}{\sqrt{N}}\right)\right| \sum_{\substack{(t, w) \in A_{N}^{x} \\
(s, v) \in A_{N}^{y}}} \mathbb{E}\left[Z_{0, t, \beta_{N}}^{A}(x, w)^{2}\right]^{1 / 2} \mathbb{E}\left[Z_{0, s, \beta_{N}}^{A}(y, v)^{2}\right]^{1 / 2} \\
& \times \sum_{N^{1-a_{N}<r<N^{1-9 a_{N} / 40}}} q_{2 r-t-s}(w-v) .
\end{aligned}
$$

By our definitions of $\sigma_{N}$ and $\beta_{N}$ in (2.15) and (1.18), we have $\sigma_{N}^{2} \log N=O(1)$. Applying Cauchy-Schwarz for the sum over $(t, w)$ and $(s, v)$, we obtain the bound

$$
\begin{aligned}
\frac{C}{N^{2}} \sum_{x, y \in I^{>}}\left|\phi\left(\frac{x}{\sqrt{N}}\right)\right|\left|\phi\left(\frac{y}{\sqrt{N}}\right)\right| & \left(\sum_{(t, w) \in A_{N}^{x}} \mathbb{E}\left[Z_{0, t, \beta_{N}}^{A}(x, w)^{2}\right] \sum_{(s, v) \in A_{N}^{y}} \mathbb{E}\left[Z_{0, s, \beta_{N}}^{A}(y, v)^{2}\right]\right)^{1 / 2} \\
\times & \sum_{N^{1-a_{N}<r<N^{1-9 a_{N} / 40}}}\left(\sum_{(t, w) \in A_{N}^{x},(s, v) \in A_{N}^{y}} q_{2 r-t-s}(w-v)^{2}\right)^{1 / 2} .
\end{aligned}
$$


We next observe that $\sum_{(t, w) \in A_{N}^{x}} \mathbb{E}\left[Z_{0, t, \beta_{N}}^{A}(x, w)^{2}\right]=\mathbb{E}\left[Z_{N, \beta_{N}}^{A}(x)^{2}\right] \leqslant C_{\hat{\beta}}$, see (4.13), (2.18) and (3.3), and similarly for the sum over $(s, v)$. This leads to the bound

$$
\frac{C C_{\hat{\beta}}}{N^{2}} \sum_{x, y \in I^{>}}\left|\phi\left(\frac{x}{\sqrt{N}}\right)\right|\left|\phi\left(\frac{y}{\sqrt{N}}\right)\right| \sum_{N^{1-a_{N}<r<N^{1-9 a_{N} / 40}}}\left(\sum_{(t, w) \in A_{N}^{x},(s, v) \in A_{N}^{y}} q_{2 r-t-s}(w-v)^{2}\right)^{1 / 2} .
$$

Since $|x-y|>N^{\frac{1}{2}-\frac{a_{N}}{10}}$ and $|x-w|,|y-v| \leqslant N^{\frac{1}{2}-\frac{a_{N}}{4}}$, we have $|w-v|>\frac{1}{2} N^{\frac{1}{2}-\frac{a_{N}}{10}}$. Given $r<N^{1-9 a_{N} / 40}$, we then have

$$
q_{2 r-t-s}(w-v)^{2} \leqslant c \exp \left(-\frac{|w-v|^{2}}{(2 r-t-s)}\right) \leqslant c \exp \left(-c N^{a_{N} / 40}\right)=\exp \left(-c e^{(\log N)^{\gamma} / 40}\right) .
$$

The sums over $(t, w),(s, v)$ and $r$ give only a polynomial factor in $N$, and hence (4.23) can be bounded by

$$
\frac{c}{N^{2}} \sum_{x, y \in I^{>}} \phi\left(\frac{x}{\sqrt{N}}\right) \phi\left(\frac{y}{\sqrt{N}}\right) \cdot N^{3} \exp \left(-c e^{(\log N)^{\gamma} / 40}\right) \underset{N \rightarrow \infty}{\longrightarrow} 0 .
$$

This proves (4.19) and completes the step.

Step (3). Let $Z_{N, \beta_{N}}^{A, B^{\geqslant}}(x)$ be defined as in (4.18) but with the constraint $r \geqslant N^{1-9 a_{N} / 40}$ instead of $r<N^{1-9 a_{N} / 40}$, i.e. with the sum over $(r, z) \in B_{N}^{\geqslant}$instead of $B_{N}$ (recall (2.10) $)$ ):

$$
Z_{N, \beta_{N}}^{A, B^{\geqslant}}(x):=\sum_{(t, w) \in A_{N}^{x},(r, z) \in B_{N}^{\geqslant}} Z_{0, t, \beta_{N}}^{A}(x, w) \cdot q_{r-t}(z-w) \cdot \sigma_{N} \xi_{r, z} \cdot Z_{r, N, \beta_{N}}(z) .
$$

In view of (4.6), we focus on the averaged quantity

$$
\frac{\sqrt{\log N}}{N} \sum_{x \in \mathbb{Z}^{2}} \frac{Z_{N, \beta_{N}}^{A, B \geqslant}(x)}{Z_{N, \beta_{N}}^{A}(x)} \phi\left(\frac{x}{\sqrt{N}}\right) .
$$

We will show that replacing in (4.24) the kernel $q_{r-t}(z-w)$ by $q_{r}(z-x)$ has a negligible effect on (4.25), in the sense that the difference tends to zero in $L^{1}(\mathbb{P})$.

We introduce the notation (recall (2.10)

$$
B_{N}^{\gtrless}(x):=\left\{(r, z) \in B_{N}^{\geqslant}:|z-x|<r^{\frac{1}{2}+\frac{a_{N}}{80}}\right\} .
$$

Recall that $g_{t}(\cdot)$ denotes the heat kernel on $\mathbb{R}^{2}$, see (1.12). By a refined local limit theorem for the simple random walk, see Theorem 2.3.11 in [LL10], we have that for $(r, z) \in B_{N}^{\geqslant}(x)$,

$$
q_{r}(z-x)=2 g_{r / 2}(z-x) \exp \left(O\left(\frac{1}{r}+\frac{|z-x|^{4}}{r^{3}}\right)\right)=2 g_{r / 2}(z-x) \exp \left(O\left(r^{-1+\frac{a_{N}}{20}}\right)\right),
$$

and similarly for $(t, w) \in A_{N}^{x}$ (see (2.3) $)$,

$q_{r-t}(z-w)=2 g_{(r-t) / 2}(z-w) \exp \left(O\left(\frac{1}{r-t}+\frac{|z-w|^{4}}{(r-t)^{3}}\right)\right)=2 g_{(r-t) / 2}(z-w) \exp \left(O\left(r^{-1+\frac{a_{N}}{20}}\right)\right)$, because $|z-w| \leqslant|z-x|+|w-x| \leqslant r^{\frac{1}{2}+\frac{a_{N}}{80}}+N^{\frac{1}{2}-\frac{a_{N}}{4}}$ and hence, for large $N$, we can bound

$$
|z-w| \leqslant 2 r^{\frac{1}{2}+\frac{a_{N}}{80}} \quad \text { and } \quad|r-t| \geqslant \frac{1}{2} r, \quad \text { for } \quad t \leqslant N^{1-a_{N}}, \quad r \geqslant N^{1-9 a_{N} / 40} .
$$


By a straightforward but tedious computation, there exists a positive constant $c$ such that

$$
\begin{array}{r}
\sup \left\{\left|\frac{g_{r / 2}(z-x)}{g_{(r-t) / 2}(z-w)}-1\right|: r>N^{1-\frac{9 a_{N}}{40}}, t<N^{1-a_{N}},|w-x|<N^{\frac{1}{2}-\frac{a_{N}}{4}}\right. \\
\left.|z-x|<r^{\frac{1}{2}+\frac{a_{N}}{80}}\right\}=O\left(e^{-c(\log N)^{\gamma}}\right)
\end{array}
$$

as $N$ tends to infinity, and by the local limit theorem, this bound can be transferred to the ratio $q_{r}(z-x) / q_{r-t}(z-w)$.

We are ready to estimate the error of replacing $q_{r-t}(z-w)$ by $q_{r}(z-x)$ in (4.24). We first restrict the sum on $(r, z) \in B_{N}^{\geqslant}(x)$. Then the contribution to (4.25) is

$$
\frac{\sqrt{\log N}}{N} \sum_{x \in \mathbb{Z}^{2}} \phi\left(\frac{x}{\sqrt{N}}\right) \sum_{\substack{(t, w) \in A_{N}^{x} \\(r, z) \in B_{N}^{\geqslant}(x)}} \frac{Z_{0, t, \beta_{N}}^{A}(x, w)}{Z_{N, \beta_{N}}^{A}(x)}\left(q_{r-t}(z-w)-q_{r}(z-x)\right) \cdot \sigma_{N} \xi_{r, z} \cdot Z_{r, N, \beta_{N}}(z),
$$

whose $L^{1}(\mathbb{P})$ norm is bounded by

$$
\begin{aligned}
& \frac{\sqrt{\log N}}{N} \sum_{x \in \mathbb{Z}^{2}}\left|\phi\left(\frac{x}{\sqrt{N}}\right)\right| \mathbb{E}\left[\frac{1}{Z_{N, \beta_{N}}^{A}(x)}\right. \\
& \left.\times\left|\sum_{\substack{(t, w) \in A_{N}^{x} \\
(r, z) \in B_{N}^{\geqslant}(x)}} Z_{0, t, \beta_{N}}^{A}(x, w)\left\{1-\frac{q_{r}(z-x)}{q_{r-t}(z-w)}\right\} q_{r-t}(z-w) \cdot \sigma_{N} \xi_{r, z} \cdot Z_{r, N, \beta_{N}}(z)\right|\right] \\
& \leqslant \frac{\sqrt{\log N}}{N} \sum_{x \in \mathbb{Z}^{2}}\left|\phi\left(\frac{x}{\sqrt{N}}\right)\right| \mathbb{E}\left[\frac{1}{Z_{N, \beta_{N}}^{A}(x)^{2}}\right]^{1 / 2} \\
& \left.\quad \times \mathbb{E}\left[\sum_{\substack{(t, w) \in A_{N}^{x} \\
(r, z) \in B_{N}^{\geqslant}(x)}} Z_{0, t, \beta_{N}}^{A}(x, w)\left\{1-\frac{q_{r}(z-x)}{q_{r-t}(z-w)}\right\} q_{r-t}(z-w) \cdot \sigma_{N} \xi_{r, z} \cdot Z_{r, N, \beta_{N}}(z)\right)^{2}\right]^{1 / 2}
\end{aligned}
$$

We recall that $\mathbb{E}\left[Z_{N, \beta_{N}}^{A}(x)^{-2}\right]$ is uniformly bounded by the negative moment estimate (3.15), while by orthogonality of terms in the chaos expansion and applying (4.27), the last expectation can be bounded as

$$
\begin{gathered}
\sum_{\substack{(t, w) \in A_{N}^{x} \\
(r, z) \in B_{N}^{\geq}(x)}} \mathbb{E}\left[Z_{0, t, \beta_{N}}^{A}(x, w)^{2}\right]\left\{1-\frac{q_{r}(z-x)}{q_{r-t}(z-w)}\right\}^{2} q_{r-t}(z-w)^{2} \sigma_{N}^{2} \mathbb{E}\left[Z_{r, N, \beta_{N}}(z)^{2}\right] \\
=O\left(e^{-c(\log N)^{\gamma}}\right)_{\substack{(t, w) \in A_{N}^{x} \\
(r, z) \in B_{N}^{\geqslant}(x)}} \mathbb{E}\left[Z_{0, t, \beta_{N}}^{A}(x, w)^{2}\right] q_{r-t}(z-w)^{2} \sigma_{N}^{2} \mathbb{E}\left[Z_{r, N, \beta_{N}}(z)^{2}\right] .
\end{gathered}
$$

By (4.24), this last sum is bounded by $\mathbb{E}\left[Z_{N, \beta_{N}}^{A, B^{*}}(0)^{2}\right] \leqslant \mathbb{E}\left[Z_{N, \beta_{N}}(0)^{2}\right] \leqslant C_{\hat{\beta}}$ uniformly in $N$, see (3.2). These estimates show that (4.29) is $O\left(\sqrt{\log N} \exp \left(-c(\log N)^{\gamma}\right)\right)$ and hence converges to zero, thus the $L^{1}(\mathbb{P})$ norm of (4.28) converges to zero too. 
To complete the step, it remains to check that in the chaos expansion (4.24) for $Z_{N, \beta_{N}}^{A, B \geqslant}(x)$, the contribution of the complementary regime $(r, z) \in B_{N}^{\geqslant} \backslash B_{N}^{\geqslant}(x)$, i.e. $|z-x| \geqslant r^{1 / 2+a_{N} / 80}$, vanishes in $L^{1}(\mathbb{P})$ as $N \rightarrow \infty$, and the same is true if we replace the kernel $q_{r-t}(z-w)$ by $q_{r}(z-x)$. Note that in this regime, by moderate deviation estimates,

$$
q_{r}(z-x) \leqslant \exp \left\{-c \frac{|z-x|^{2}}{r}\right\} \leqslant \exp \left\{-c r^{a_{N} / 40}\right\} \leqslant \exp \left\{-c e^{c(\log N)^{\gamma}}\right\},
$$

and the same bound holds for $q_{r-t}(z-w)$, because $|w-x| \leqslant N^{\frac{1}{2}-\frac{a_{N}}{4}}=o\left(r^{1 / 2}\right)$ as $N \rightarrow \infty$ (since $r \geqslant N^{1-9 a_{N} / 40}$ ) and hence $|w-x|=o(|z-x|)$ in this regime. These bounds can then be used to show that

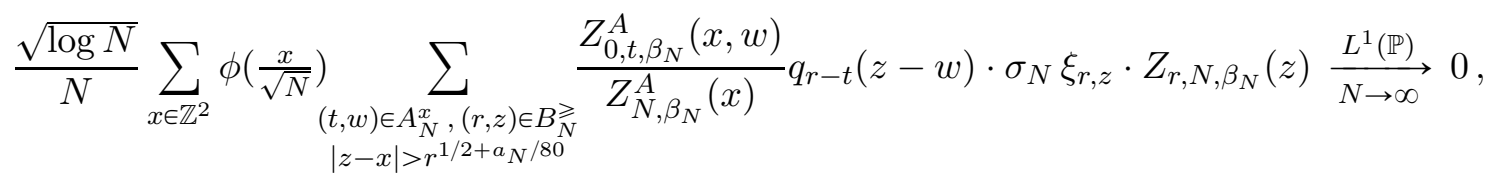

and the same holds when $q_{r-t}(z-w)$ is replaced by $q_{r}(z-x)$. Indeed, we can argue as in (4.29) and then use the fact that the number of terms in the sums over $x,(t, w),(r, z)$ is only polynomial in $N$, while (4.30) decays faster.

4.4. Proof of Proposition 2.4. Recalling (2.14), we want to prove that

$$
\frac{\sqrt{\log N}}{\sqrt{\pi} \hat{\beta}} \frac{1}{N} \sum_{x \in \mathbb{Z}^{2}}\left(Z_{N, \beta_{N}}^{B \geqslant}(x)-1\right) \phi\left(\frac{x}{\sqrt{N}}\right) \underset{N \rightarrow \infty}{\stackrel{d}{\longrightarrow}}\left\langle v^{\left(\sqrt{2} c_{\hat{\beta}}\right)}(1 / 2, \cdot), \phi\right\rangle,
$$

where $v^{(c)}(s, x)$ is the solution of the two-dimensional additive SHE as in (1.11).

The proof of (4.31) follows the same line as the proof of Theorem 2.13 in CSZ17b, which proved the convergence of the fluctuations of the polymer partition function $Z_{N, \beta_{N}}(x)$ as a space-time random field to the solution of the additive SHE. To see heuristically why the limit in (4.31) should be Gaussian, we can write the LHS of (4.31) as a polynomial chaos expansion, see (4.35), where the dominant contribution (in $L^{2}$ ) comes from terms of finite order in the expansion because $\hat{\beta} \in(0,1)$. Each such term is of the form $\sigma_{N}^{k} \prod_{i=1}^{k} q_{n_{i}-n_{i-1}}\left(x_{i}-x_{i-1}\right) \xi_{n_{i}, x_{i}}$, which due to the random walk transition kernels $q .(\cdot)$, depends only on disorder $\xi_{, .}$. in a neighborhood of $\left(n_{0}, x_{0}\right):=(0, x)$ that is negligible on the diffusive scale. Given such local dependence on the disorder, it is then not surprising that when averaged over $(0, x)$ on the diffusive scale with weight $\phi(x / \sqrt{N})$, we should get a Gaussian limit. The proof in CSZ17b also shows that terms of order two and higher in the chaos expansion leads to an independent white noise in the limit, which leads to a noise coefficient $c_{\hat{\beta}}>1$ in (1.11).

We now recall the key element in the proof of Theorem 2.13 in [CSZ17b and show how it can be adapted to our setting. The key technical tool is the following variant of Proposition 8.1 in [CSZ17b], specialized to the simple random walk on $\mathbb{Z}^{2}$ (where we average in space, rather than in space-time). It will show that, in the polynomial chaos expansion of the left hand side of (4.31), there are "building blocks" that converge in distribution to independent Gaussian random variables.

Proposition 4.1. For integer $M$ and $i \in\{1, \ldots, M\}$, define intervals $I_{i}:=\left(N^{\frac{i-1}{M}}, N^{\frac{i}{M}}\right] . A$ $k$-tuple $\left(i_{1}, \ldots, i_{k}\right) \in\{1, \ldots, M\}^{k}$ is said to belong to $\{1, \ldots, M\}_{\sharp}^{k}$ if $\left|i_{j}-i_{j^{\prime}}\right| \geqslant 2$ for all $j \neq j^{\prime}$. For $N \in \mathbb{N}$, let $\xi=\left(\xi_{n, x}^{(N)}\right)_{(n, x) \in \mathbb{N} \times \mathbb{Z}^{2}}$ be i.i.d. with zero mean and unit variance. 
Given $N, M \in \mathbb{N}$, a $k$-tuple $\left(i_{1}, \ldots, i_{k}\right) \in\{1, \ldots, M\}_{\sharp}^{k}$ and a point $x \in \mathbb{Z}^{2}$, we define a random variable $\Theta_{i_{1}, \ldots, i_{k}}^{N ; M}(x)$, a multilinear polynomial of degree $k$ in the variables $\xi$ 's, as follows:

$$
\Theta_{i_{1}, \ldots, i_{k}}^{N ; M}(x):=\left(\frac{M}{R_{N}}\right)^{\frac{k-1}{2}} \sum_{\substack{n_{1} \in I_{i_{1}}, n_{2}-n_{1} \in I_{i_{2}}, \ldots, n_{k}-n_{k-1} \in I_{i} \\ n_{0}:=0, x_{0}:=x, x_{1}, \ldots, x_{k} \in \mathbb{Z}^{d}}} \prod_{j=1}^{k} q_{n_{j}-n_{j-1}}\left(x_{j}-x_{j-1}\right) \prod_{i=1}^{k} \xi_{n_{i}, x_{i}},
$$

where $q_{n}(x)$ is the transition kernel of the simple symmetric random walk on $\mathbb{Z}^{2}$, and $R_{N}$ is the expected overlap, defined in (1.17). For $\phi \in C_{c}\left(\mathbb{R}^{2}\right)$, we define the space-averaged version

$$
\Theta_{i_{1}, \ldots, i_{k}}^{N ; M ; \phi}:=\frac{1}{N} \sum_{x \in \mathbb{Z}^{2}} \Theta_{i_{1}, \ldots, i_{k}}^{N ; M}(x) \phi\left(\frac{x}{\sqrt{N}}\right)
$$

Let $D_{M}$ denote the subset of $\left(i_{1}, \ldots, i_{k}\right) \in\{1, \ldots, M\}_{\sharp}^{k}$ that satisfy $i_{1}>\max \left\{i_{2}, \ldots, i_{k}\right\}$, called dominated sequences. Then, for any fixed $M \in \mathbb{N}$ and $\phi \in C_{c}\left(\mathbb{R}^{2}\right)$, the family of random variables $\left(\Theta_{\left(i_{1}, \ldots, i_{k}\right)}^{\left.N ; M ;)_{(i, ~} ; \ldots, i_{k}\right) \in D_{M}}\right.$ converges in distribution as $N \rightarrow \infty$ to a family $\left(\zeta_{\left(i_{1}, \ldots, i_{k}\right)}^{\phi}\right)_{\left(i_{1}, \ldots, i_{k}\right) \in D_{M}}$ of independent Gaussian random variables with

$$
\mathbb{E}\left[\zeta_{\left(i_{1}, \ldots, i_{k}\right)}^{\phi}\right]=0, \quad \mathbb{V a r}\left[\zeta_{\left(i_{1}, \ldots, i_{k}\right)}^{\phi}\right]=2 \sigma_{\phi}^{2} \mathbb{1}_{\left\{i_{1}=M\right\}},
$$

i.e. the variance is non-zero only if $i_{1}=M$, and is given by

$$
\sigma_{\phi}^{2}=\int_{\left(\mathbb{R}^{2}\right)^{2}} \phi(x) K_{\frac{1}{2}}(x, y) \phi(y) \mathrm{d} x \mathrm{~d} y \quad \text { with } \quad K_{\frac{1}{2}}(x, y)=\int_{0}^{\frac{1}{2}} \frac{1}{4 \pi u} e^{-\frac{|x-y|^{2}}{4 u}} \mathrm{~d} u .
$$

The proof of Proposition 4.1] in [CSZ17b] is based on a variant of the fourth-moment theorem for polynomial chaos expansions, as formulated in [CSZ17b, Theorem 4.2], which was obtained in [NPR10] building on [NP05, dJ87, dJ90]. To check the variance, note that

$$
\begin{aligned}
\operatorname{Var}\left[\Theta_{i_{1}, \ldots, i_{k}}^{N ; M ; \phi}\right]=\frac{1}{N^{2}} & \sum_{x, y \in \mathbb{Z}^{2}} \sum_{n_{1} \in I_{i_{1}}, x_{1} \in \mathbb{Z}^{2}} \phi\left(\frac{x}{\sqrt{N}}\right) \phi\left(\frac{y}{\sqrt{N}}\right) q_{n_{1}}\left(x_{1}-x\right) q_{n_{1}}\left(x_{1}-y\right) \\
& \times\left(\frac{M}{R_{N}}\right)^{k-1} \sum_{\substack{n_{2}-n_{1} \in I_{i_{2}}, \ldots, n_{k}-n_{k-1} \in I_{i_{k}} \\
x_{2}, \ldots, x_{k} \in \mathbb{Z}^{2}}} \prod_{j=2}^{k} q_{n_{j}-n_{j-1}}\left(x_{j}-x_{j-1}\right)^{2},
\end{aligned}
$$

where the second line tends to 1 as $N \rightarrow \infty$ by the definition of $R_{N}$ and $I_{i}$. We can write

$$
\sum_{x_{1} \in \mathbb{Z}^{2}} q_{n_{1}}\left(x_{1}-x\right) q_{n_{1}}\left(x_{1}-y\right)=q_{2 n_{1}}(x-y) \underset{n_{1} \rightarrow \infty}{=}\left(g_{n_{1}}(x-y)+o\left(\frac{1}{n_{1}}\right)\right) 2 \mathbb{1}_{\left\{x-y \in \mathbb{Z}_{\text {even }}^{2}\right\}}
$$

by the local limit theorem, where $\mathbb{Z}_{\text {even }}^{2}:=\left\{(a, b) \in \mathbb{R}^{2}: a+b\right.$ is even $\}, g_{t}(x)$ is as in (1.12), the factor 2 is due to random walk periodicity and we have $g_{n_{1}}(\cdot)$ instead of $g_{2 n_{1}}(\cdot)$ because the random walk $S_{n}$ has covariance matrix $\frac{n}{2} I$. Then, by a Riemann sum approximation, as $N \rightarrow \infty$ the first line in (4.34) is close to the integral

$$
\int_{\left(\mathbb{R}^{2}\right)^{2}} \phi\left(x^{\prime}\right) \phi\left(y^{\prime}\right)\left(\int_{N}^{N \frac{i_{1}-1}{M}-1} g_{u}\left(x^{\prime}-y^{\prime}\right) \mathrm{d} u\right) \mathrm{d} x^{\prime} \mathrm{d} y^{\prime} \underset{N \rightarrow \infty}{\longrightarrow}\left\{\begin{array}{ll}
0 & \text { if } i_{1}<M \\
2 \sigma_{\phi}^{2} & \text { if } i_{1}=M
\end{array},\right.
$$


with $\sigma_{\phi}^{2}$ defined in (4.33). Also note that for $i_{1}=M$, the dominant contribution comes from $n_{1} \in[\varepsilon N, N]$ for $\varepsilon$ small, and hence restricting to $n_{1} \in[1, N]$, or $n_{1} \in I_{M}=\left(N^{1-\frac{1}{M}}, N\right]$, or $n_{1} \geqslant N^{1-9 a_{N} / 40}$ makes no difference as $N \rightarrow \infty$ (for any fixed $M \in \mathbb{N}$ ).

Let us show how Proposition 4.1 can be applied to prove (4.31). Recall from (2.19) that

$$
Z_{N, \beta_{N}}^{B \geqslant}(x)-1=\sum_{k=1}^{N} \sigma_{N}^{k} \sum_{\substack{N^{1-9 a_{N} / 40}<n_{1}<\ldots<n_{k} \leqslant N \\ n_{0}:=0, x_{0}:=x, x_{1}, \ldots, x_{k} \in \mathbb{Z}^{2}}} \prod_{i=1}^{k} q_{n_{i}-n_{i-1}}\left(x_{i}-x_{i-1}\right) \xi_{n_{i}, x_{i}} .
$$

For fixed $M \in \mathbb{N}$, grouping each $n_{i}-n_{i-1}$ according to which interval $I_{j}:=\left(N^{\frac{j-1}{M}}, N^{\frac{j}{M}}\right]$ it belongs to, and recalling (2.15) and (1.18), we have the following approximation:

$$
\frac{\sqrt{\log N}}{\sqrt{\pi} \hat{\beta}} \frac{1}{N} \sum_{x \in \mathbb{Z}^{2}}\left(Z_{N, \beta_{N}}^{B \geqslant}(x)-1\right) \phi\left(\frac{x}{\sqrt{N}}\right) \approx \sum_{k=1}^{M} \frac{\hat{\beta}^{k-1}}{M^{(k-1) / 2}} \sum_{\substack{\left(i_{1}, \ldots, i_{k}\right) \in\{1, \ldots, M\}_{\sharp}^{k} \\ i_{1}=M}} \Theta_{i_{1}, \ldots, i_{k}}^{N ; M ; \phi},
$$

where $\approx$ means that the difference of the two sides vanishes in $L^{2}(\mathbb{P})$ as $N \rightarrow \infty$ followed by $M \rightarrow \infty$. The restriction $i_{1}=M$ in (4.36) is due to $n_{1}>N^{1-9 a_{N} / 40}$, which gives rise to a dominated sequence. The error from relaxing $n_{1}>N^{1-9 a_{N} / 40}$ to $n_{1} \in I_{M}=\left(N^{\frac{M-1}{M}}, N\right]$ is negligible in $L^{2}$, as noted above, while the error from restricting to $\left(i_{1}, \ldots, i_{k}\right) \in\{1, \ldots, M\}_{\sharp}^{k}$ (rather than the whole $\{1, \ldots, M\}^{k}$ ) is also negligible in $L^{2}(\mathbb{P})$, when we first send $N \rightarrow \infty$ and then $M \rightarrow \infty$, as shown in [CSZ17b, Lemma 6.2].

We can then apply Proposition 4.1 to conclude that, as we let $N \rightarrow \infty$ for fixed $M \in \mathbb{N}$, the right hand side of (4.36) converges in distribution to the same expression with $\Theta_{i_{1}, \ldots, i_{k}}^{N ; M ;}$ replaced by $\zeta_{i_{1}, \ldots, i_{k}}^{\phi}$, i.e. to a Gaussian random variable with zero mean and with variance

$$
\sum_{k=1}^{M} \frac{\left(\hat{\beta}^{2}\right)^{k-1}}{M^{k-1}} 2 \sigma_{\phi}^{2} \cdot\left|\left\{\left(i_{1}, \ldots, i_{k}\right) \in\{1, \ldots, M\}_{\sharp}^{k}: i_{1}=M\right\}\right| .
$$

If we let $M \rightarrow \infty$, since $\left|\left\{\left(i_{1}, \ldots, i_{k}\right) \in\{1, \ldots, M\}_{\sharp}^{k}: i_{1}=M\right\}\right|=M^{k-1}(1+o(1))$, the sum in (4.37) converges to the following explicit expression, with $c_{\hat{\beta}}$ as in (1.11):

$$
2 \sigma_{\phi}^{2} \sum_{k \geqslant 1} \hat{\beta}^{2(k-1)}=2 \sigma_{\phi}^{2} \frac{1}{1-\hat{\beta}^{2}}=\left(\sqrt{2} c_{\hat{\beta}}\right)^{2} \sigma_{\phi}^{2}
$$

This agrees with the variance of $\left\langle v^{\left(\sqrt{2} c_{\hat{\beta}}\right)}(1 / 2, \cdot), \phi\right\rangle$, see (1.13), which proves (4.31).

\section{Edwards-Wilkinson Fluctuations for KPZ}

In this section we prove Theorem 1.1, which gives Edwards-Wilkinson fluctuations for the Hopf-Cole solution $h_{\varepsilon}(t, z)=\log u_{\varepsilon}(t, z)$ of the mollified KPZ (where $u_{\varepsilon}(t, z)$ solves the mollified SHE, see (1.5)).

The proof follows the same lines as in the directed polymer case. This is possible because $u^{\varepsilon}(t, z)$ admits a Feynman-Kac representation, which casts it in a form close to the directed polymer partition function of size $N=\varepsilon^{-2} t$. Indeed, by [BC95, Section 3] (see also [CSZ17b, 
eq. (2.27)]), for fixed $(t, z)$ we have the following equality in law:

$$
\begin{aligned}
u^{\varepsilon}(t, z) & \stackrel{d}{=} \mathrm{E}_{\varepsilon^{-1} z}\left[\exp \left\{\iint_{\left(0, \varepsilon^{-2} t\right) \times \mathbb{R}^{2}}\left(\beta_{\varepsilon} j\left(B_{s}-x\right) \xi(s, x) \mathrm{d} s \mathrm{~d} x-\frac{1}{2} \beta_{\varepsilon}^{2} j\left(B_{s}-x\right)^{2} \mathrm{~d} s \mathrm{~d} x\right)\right\}\right] \\
& =\mathrm{E}_{\varepsilon^{-1} z}\left[\exp \left\{\int_{0}^{\varepsilon^{-2} t} \int_{\mathbb{R}^{2}} \beta_{\varepsilon} j\left(B_{s}-x\right) \xi(s, x) \mathrm{d} s \mathrm{~d} x-\frac{1}{2} \beta_{\varepsilon}^{2}\left(\varepsilon^{-2} t\right)\|j\|_{L^{2}\left(\mathbb{R}^{2}\right)}^{2}\right\}\right],
\end{aligned}
$$

where $B=\left(B_{s}\right)_{s \in[0, \infty)}$ under $\mathrm{P}_{x}$ is a standard Brownian motion on $\mathbb{R}^{2}$ started at $x$.

We first perform a decomposition of $u^{\varepsilon}(t, z)$ similar to that described described in Section 2, which reduces Theorem 1.1 to the four Propositions 5.15.4 (see \$5.1). These are proved later (see \$5.3) in analogy with the corresponding results for directed polymer (see Section 4), exploiting moment bounds analogous to those in Section 3 (see \$5.2).

Henceforth we set $t=1$ and we focus on $u^{\varepsilon}(z):=u^{\varepsilon}(1, z)$.

5.1. Decomposition, linearization and Wiener chaos. By (5.1) and (1.21)(1.22), $u^{\varepsilon}(z)$ is comparable to $Z_{N}(x)$, provided we identify $N=\varepsilon^{-2}, x=\varepsilon^{-1} z$.

As in (2.2)-(2.3) , we define (for a $\gamma^{*}$ small enough, depending only on $\hat{\beta}$ as in (2.2) $)$

$$
\begin{gathered}
a_{\varepsilon}:=\frac{1}{\left(\log \varepsilon^{-2}\right)^{1-\gamma}} \quad \text { for fixed } \gamma \in\left(0, \gamma^{*}\right), \\
A_{\varepsilon}^{z}:=\left\{(s, x): 0<s \leqslant\left(\varepsilon^{-2}\right)^{1-a_{\varepsilon}},\left|x-\varepsilon^{-1} z\right|<\left(\varepsilon^{-2}\right)^{\frac{1}{2}-\frac{a_{\varepsilon}}{4}}\right\},
\end{gathered}
$$

and we introduce a modified partition function $u_{A}^{\varepsilon}(z)$, obtained by restricting the double integral in the first line of (5.1) to the set $(s, x) \in A_{\varepsilon}^{z}$. This yields the decomposition

$$
u^{\varepsilon}(z)=u_{A}^{\varepsilon}(z)+\hat{u}_{A}^{\varepsilon}(z),
$$

where $\hat{u}_{A}^{\varepsilon}(z)$, defined by this relation, is a "remainder" which, for fixed $z$, can be shown to be much smaller than $u_{A}^{\varepsilon}(z)$. More precisely, as in (2.6) -(2.7), we define $O^{\varepsilon}(z)$ by

$$
\log u^{\varepsilon}(z)=\log u_{A}^{\varepsilon}(z)+\frac{\hat{u}_{A}^{\varepsilon}(z)}{u_{A}^{\varepsilon}(z)}+O^{\varepsilon}(z)
$$

and we have the following analogues of Propositions 2.1.2.2.

Proposition 5.1. Let $O^{\varepsilon}(\cdot)$ be defined as above, then for any $\phi \in C_{c}\left(\mathbb{R}^{2}\right)$

$$
\sqrt{\log \varepsilon^{-1}} \int_{\mathbb{R}^{2}}\left(O^{\varepsilon}(z)-\mathbb{E}\left[O^{\varepsilon}(z)\right]\right) \phi(z) \mathrm{d} z \underset{\varepsilon \downarrow 0}{\stackrel{L^{2}(\mathbb{P})}{\longrightarrow}} 0 .
$$

Proposition 5.2. Let $u_{A}^{\varepsilon}(\cdot)$ be defined as above, then for any $\phi \in C_{c}\left(\mathbb{R}^{2}\right)$

$$
\sqrt{\log \varepsilon^{-1}} \int_{\mathbb{R}^{2}}\left(\log u_{A}^{\varepsilon}(z)-\mathbb{E}\left[\log u_{A}^{\varepsilon}(z)\right]\right) \phi(z) \mathrm{d} z \underset{\varepsilon \downarrow 0}{\stackrel{L^{2}(\mathbb{P})}{\longrightarrow}} 0 .
$$

Next, in analogy with (2.10)-(2.11), we introduce the subset

$$
B_{\varepsilon}^{\geqslant}:=\left(\left(\varepsilon^{-2}\right)^{1-9 a_{\varepsilon} / 40}, \varepsilon^{-2}\right) \times \mathbb{R}^{2},
$$

and we introduce $u_{B \geqslant}^{\varepsilon}(z)$, obtained by restricting the double integral in the first line of (5.1) to the set $(s, x) \in B_{\varepsilon}^{\geqslant}$. We have the following analogues of Propositions 2.3 2.4. 
Proposition 5.3. Let $u_{A}^{\varepsilon}(\cdot), \hat{u}_{A}^{\varepsilon}(\cdot), u_{B \geqslant}^{\varepsilon}(\cdot)$ be defined as above, then for any $\phi \in C_{c}\left(\mathbb{R}^{2}\right)$

$$
\sqrt{\log \varepsilon^{-1}} \int_{\mathbb{R}^{2}}\left(\frac{\hat{u}_{A}^{\varepsilon}(z)}{u_{A}^{\varepsilon}(z)}-\left(u_{B \geqslant}^{\varepsilon}(z)-1\right)\right) \phi(z) \mathrm{d} z \stackrel{L^{1}(\mathbb{P})}{\underset{\varepsilon \downarrow 0}{\longrightarrow}} 0 .
$$

Proposition 5.4. Let $u_{B \geqslant}^{\varepsilon}(\cdot)$ be defined as above, then for any $\phi \in C_{c}\left(\mathbb{R}^{2}\right)$

$$
\frac{\sqrt{\log \varepsilon^{-1}}}{\sqrt{2 \pi} \hat{\beta}} \int_{\mathbb{R}^{2}}\left(u_{B \geqslant}^{\varepsilon}(z)-1\right) \phi(z) \underset{\varepsilon \downarrow 0}{\stackrel{d}{\longrightarrow}}\left\langle v^{\left(c_{\hat{\beta}}\right)}(1, \cdot), \phi\right\rangle .
$$

Theorem 1.1 is a direct consequence of Propositions 5.1 5.4. Regarding the centering, by (5.5) we have $\mathbb{E}\left[\log u^{\varepsilon}(z)\right]=\mathbb{E}\left[\log u_{A}^{\varepsilon}(z)\right]+\mathbb{E}\left[O^{\varepsilon}(z)\right]$, because $\hat{u}_{A}^{\varepsilon}(z) / u_{A}^{\varepsilon}(z)$ has zero mean, as we show in a moment.

By (5.1) and the definition of Wick exponential [J97, §3.2], we have the following Wiener chaos representation for $u^{\varepsilon}(z)$, where we set $t_{0}:=0$ and $y_{0}:=\varepsilon^{-1} z$ :

$$
u^{\varepsilon}(z) \stackrel{d}{=} 1+\sum_{k \geqslant 1} \beta_{\varepsilon}^{k} \int_{\substack{0<t_{1}<\cdots<t_{k}<\varepsilon^{-2} \\ \vec{x} \in\left(\mathbb{R}^{2}\right)^{k}}}\left(\int_{\left(\mathbb{R}^{2}\right)^{k}} \prod_{i=1}^{k} g_{t_{i}-t_{i-1}}\left(y_{i}-y_{i-1}\right) j\left(y_{i}-x_{i}\right) \mathrm{d} \vec{y}\right) \prod_{i=1}^{k} \xi\left(t_{i}, x_{i}\right) \mathrm{d} t_{i} \mathrm{~d} x_{i},
$$

where $g_{t}(\cdot)$ is the transition kernel of the Brownian motion.

The modified partition function $u_{A}^{\varepsilon}(z)$ admits a similar Wiener chaos expansions, with the outer integrals restricted to the set $\left\{\left(t_{1}, x_{1}\right), \ldots,\left(t_{k}, x_{k}\right)\right\} \subseteq A_{\varepsilon}^{z}$. It follows that the Wiener chaos expansion of $\hat{u}_{A}^{\varepsilon}(z):=u^{\varepsilon}(z)-u_{A}^{\varepsilon}(z)$ contains at least one factor $\xi\left(t_{i}, x_{i}\right)$ with $\left(t_{i}, x_{i}\right)$ outside $A_{z}^{\varepsilon}$, which is not present in $u_{A}^{\varepsilon}(z)$, hence $\mathbb{E}\left[\hat{u}_{A}^{\varepsilon}(z) / u_{A}^{\varepsilon}(z)\right]=0$.

Similarly, the Wiener chaos expansions of $u_{B \geqslant}^{\varepsilon}(z)$ is obtained by restricting the outer integrals in (5.9) to the set $\left\{\left(t_{1}, x_{1}\right), \ldots,\left(t_{k}, x_{k}\right)\right\} \subseteq B_{\varepsilon}^{\geqslant}$, i.e. imposing $t_{1}>\left(\varepsilon^{-2}\right)^{1-9 a_{\varepsilon} / 40}$.

5.2. Moment bounds. We estimate positive and negative moments of $u^{\varepsilon}(z)$.

We start with the second moment. We prove below the following bounds for $u^{\varepsilon}(z), u_{A}^{\varepsilon}(z)$ and $\hat{u}_{A}^{\varepsilon}(z)$, which are close analogues of (3.2), (3.3), (3.4):

$$
\begin{aligned}
& \forall \hat{\beta} \in(0,1) \quad \exists C_{\hat{\beta}}<\infty \text { such that } \forall \varepsilon>0: \\
& \mathbb{E}\left[u^{\varepsilon}(z)^{2}\right] \leqslant C_{\hat{\beta}}, \quad \mathbb{E}\left[u_{A}^{\varepsilon}(z)^{2}\right] \leqslant C_{\hat{\beta}}, \quad \mathbb{E}\left[\hat{u}_{A}^{\varepsilon}(z)^{2}\right] \leqslant C_{\hat{\beta}} a_{\varepsilon} .
\end{aligned}
$$

We can now easily deduce bounds for higher positive moments. By hypercontractivity [J97, Theorem 5.1], the $L^{p}$ norm of a Wiener chaos expansion like (5.9) is bounded by the $L^{2}$ norm of a modified expansion, with the $k$-th order term multiplied by $\left(c_{p}\right)^{k}$ (i.e., $\hat{\beta}$ replaced by $\left.c_{p} \hat{\beta}\right)$, with $c_{p}:=\sqrt{p-1}$. For $\hat{\beta} \in(0,1)$ we can choose $p>2$ such that $\hat{\beta} c_{p}<1$, so as to apply the bounds in (5.10). This yields an analogue of (3.12):

$$
\begin{aligned}
& \forall \hat{\beta} \in(0,1) \quad \exists p=p_{\hat{\beta}} \in(2, \infty) \quad \exists C_{\hat{\beta}}^{\prime}<\infty \text { such that } \forall \varepsilon>0: \\
& \mathbb{E}\left[u^{\varepsilon}(z)^{p}\right] \leqslant C_{\hat{\beta}}^{\prime}, \quad \mathbb{E}\left[u_{A}^{\varepsilon}(z)^{p}\right] \leqslant C_{\hat{\beta}}^{\prime}, \quad \mathbb{E}\left[\left|\hat{u}_{A}^{\varepsilon}(z)\right|^{p}\right] \leqslant C_{\hat{\beta}}^{\prime}\left(a_{\varepsilon}\right)^{p / 2} .
\end{aligned}
$$

Proof of (5.10). We compute $\mathbb{E}\left[u^{\varepsilon}(z)^{2}\right]$ by using (5.9), applying the identity $g_{t}(y) g_{t}\left(y^{\prime}\right)=$ $4 g_{2 t}\left(y-y^{\prime}\right) g_{2 t}\left(y+y^{\prime}\right)$, and switching to new variables $z_{i}:=y_{i}-y_{i}^{\prime}, w_{i}:=y_{i}+y_{i}^{\prime}$. This leads 
to the following expression (see [CSZ18, §8.2] for details):

$$
\begin{array}{r}
\mathbb{E}\left[u^{\varepsilon}(z)^{2}\right]=1+\sum_{k \geqslant 1}\left(\beta_{\varepsilon}^{2}\right)^{k} \int_{\substack{0<t_{1}<\ldots<t_{k}<\varepsilon^{-2} \\
\vec{z} \in\left(\mathbb{R}^{2}\right)^{k}, \vec{w} \in\left(\mathbb{R}^{2}\right)^{k}}}\left(\prod_{i=1}^{k} g_{2\left(t_{i}-t_{i-1}\right)}\left(z_{i}-z_{i-1}\right) J\left(z_{i}\right) .\right. \\
\left.\cdot g_{2\left(t_{i}-t_{i-1}\right)}\left(w_{i}-w_{i-1}\right)\right) \prod_{i=1}^{k} \mathrm{~d} t_{i} \mathrm{~d} z_{i} \mathrm{~d} w_{i},
\end{array}
$$

where $J:=j * j$ and we set $z_{0}:=0, w_{0}:=2 \varepsilon^{-1} z$. Integrating out $w_{k}, w_{k-1}, \ldots, w_{1}$, we get

$$
\mathbb{E}\left[u^{\varepsilon}(z)^{2}\right]=1+\sum_{k \geqslant 1}\left(\beta_{\varepsilon}^{2}\right)^{k} \int_{\substack{0<t_{1}<\cdots<t_{k}<\varepsilon^{-2} \\ \vec{z} \in\left(\mathbb{R}^{2}\right)^{k}}}\left(\prod_{i=1}^{k} g_{2\left(t_{i}-t_{i-1}\right)}\left(z_{i}-z_{i-1}\right) J\left(z_{i}\right)\right) \prod_{i=1}^{k} \mathrm{~d} t_{i} \mathrm{~d} z_{i} .
$$

We recall that $j$, hence $J$, has compact support. If we define

$$
\bar{r}(t):=\sup _{z^{\prime} \in \operatorname{supp}(J)} \int_{\mathbb{R}^{2}} g_{2 t}\left(z-z^{\prime}\right) J(z) \mathrm{d} z,
$$

we can bound

$$
\mathbb{E}\left[u^{\varepsilon}(z)^{2}\right] \leqslant 1+\sum_{k \geqslant 1}\left(\beta_{\varepsilon}^{2}\right)^{k} \int_{0<t_{1}<\cdots<t_{k}<\varepsilon^{-2}} \ldots \int_{i=1}^{k} \bar{r}\left(t_{i}-t_{i-1}\right) \mathrm{d} t_{i} \leqslant 1+\sum_{k \geqslant 1}\left\{\beta_{\varepsilon}^{2} \int_{0}^{\varepsilon^{-2}} \bar{r}(t) \mathrm{d} t\right\}^{k} .
$$

Note that $\bar{r}(\cdot)$ is bounded for $t \geqslant 0$ and it satisfies $\bar{r}(t)=\frac{1}{4 \pi t}+O(1)$ as $t \rightarrow \infty$, by (1.12). Recalling (1.2), we see that the bracket converges to $\hat{\beta}^{2}$ as $\varepsilon \rightarrow 0$, hence the series is uniformly bounded for $\hat{\beta}<1$. This proves the first bound in (5.10).

The second bound in (5.10) follows because $\mathbb{E}\left[u_{A}^{\varepsilon}(z)^{2}\right] \leqslant \mathbb{E}\left[u^{\varepsilon}(z)^{2}\right]$, since the Wiener chaos expansion for $u_{A}^{\varepsilon}(z)$ is a subset of the expansion for $u^{\varepsilon}(z)$.

Finally, the third bound in (5.10) can be proved similarly to (3.4) (see Subsection 3.4), because $\mathbb{E}\left[\hat{u}_{A}^{\varepsilon}(z)^{2}\right]$ can be bounded by an expression analogous to (5.12) 1

We next estimate negative moments, establishing the following analogues of (3.14)-(3.16):

$$
\begin{aligned}
\forall \hat{\beta} \in & (0,1) \quad \forall p \in(0, \infty) \quad \exists C_{p, \hat{\beta}}<\infty \text { such that } \forall \varepsilon>0: \\
& \mathbb{E}\left[u^{\varepsilon}(z)^{-p}\right] \leqslant C_{p, \hat{\beta}}<\infty, \\
& \mathbb{E}\left[u_{A}^{\varepsilon}(z)^{-p}\right] \leqslant C_{p, \hat{\beta}}<\infty, \\
& \mathbb{E}\left[\left|\log u_{A}^{\varepsilon}(z)\right|^{p}\right] \leqslant C_{p, \hat{\beta}}<\infty .
\end{aligned}
$$

Since (5.15) follows easily from (5.14), it suffices to prove (5.13)-(5.14). These are direct corollaries of the following result, analogous to Proposition 3.1 .

\footnotetext{
${ }^{\dagger}$ Note that $\hat{u}_{A}^{\varepsilon}(z)$ contains at least one point $\left(t_{i}, x_{i}\right)$ outside $A_{\varepsilon}^{z}$ in the Wiener chaos representation (5.9). Since $j(\cdot)$ has compact support, say included in the ball $B_{r}:=\left\{x \in \mathbb{R}^{2}:|x| \leqslant r\right\}$, the corresponding point $\left(t_{i}, y_{i}\right)$ in (5.9) must be close to (i.e. at distance at most $r$ from) the point $\left(t_{i}, x_{i}\right)$. Then $\mathbb{E}\left[\hat{u}_{A}^{\varepsilon}(z)^{2}\right]$ can be bounded by an expression analogous to (5.12), with the integrals restricted to the set where at least one point $\left(t_{i}, \frac{1}{2} w_{i}\right)=\left(t_{i}, \frac{1}{2}\left(y_{i}+y_{i}^{\prime}\right)\right)$ is close to $\left(A_{\varepsilon}^{z}\right)^{c}$. This allows to follow the proof in Subsection 3.4
} 
Proposition 5.5 (Left tail for KPZ). For $\Lambda \subseteq\left(0, \varepsilon^{-2}\right) \times \mathbb{R}^{2}$, denote by $u_{\Lambda}^{\varepsilon}(z)$ what we obtain by restricting the double integral in the first line of (5.1) to $(s, x) \in \Lambda$, i.e.

$$
u_{\Lambda}^{\varepsilon}(z):=\mathrm{E}_{\varepsilon^{-1} z}\left[\exp \left\{\iint_{(s, x) \in \Lambda}\left(\beta_{\varepsilon} j\left(B_{s}-x\right) \xi(s, x) \mathrm{d} s \mathrm{~d} x-\frac{1}{2} \beta_{\varepsilon}^{2} j\left(B_{s}-x\right)^{2} \mathrm{~d} s \mathrm{~d} x\right)\right\}\right] .
$$

For any $\hat{\beta} \in(0,1)$ there is $c_{\hat{\beta}} \in(0, \infty)$ with the following property: for any $\varepsilon>0$ and for any choice of subset $\Lambda \subseteq\left(0, \varepsilon^{-2}\right) \times \mathbb{R}^{2}$, one has

$$
\forall t \geqslant 0: \quad \mathbb{P}\left(\log u_{\Lambda}^{\varepsilon}(z) \leqslant-t\right) \leqslant c_{\hat{\beta}} e^{-t^{2} / c_{\hat{\beta}}} .
$$

It remains to prove Proposition 5.5. We first need to recall concentration inequalities for white noise (see Appendix $\mathrm{C}$ for more details).

The white noise $\xi=(\xi(s, y))_{(s, y) \in[0, \infty) \times \mathbb{R}^{2}}$ can be viewed as a random element of a separable Banach space $E$ of distributions on $[0, \infty) \times \mathbb{R}^{2}$ (e.g. a negative Hölder space, see [CD18]). Its law $\mu$ is the Gaussian measure on $E$ with Cameron-Martin space $H=$ $L^{2}\left([0, \infty) \times \mathbb{R}^{2}\right)$, and the triple $(H, E, \mu)$ is a so-called abstract Wiener space. In this setting, sharp concentration inequalities are known to hold for (not necessarily convex) functions $f: E \rightarrow \mathbb{R}$ that are Lipschitz in the directions of $H$, see [Led96, eq. (4.7) and (4.8)].

We need to work with convex functions $f: E \rightarrow \mathbb{R} \cup\{-\infty,+\infty\}$ that are not globally Lipschitz. Remarkably, such functions still enjoy concentration inequalities for the left tail (but not, in general, for the right tail). For $x \in E$ with $|f(x)|<\infty$, denote by $|\nabla f(x)| \in[0, \infty]$ the maximal gradient of $f$ in the directions of $H$, defined by

$$
|\nabla f(x)|:=\sup _{h \in H:\|h\|_{H} \leqslant 1} \lim _{\delta \downarrow 0} \frac{|f(x+\delta h)-f(x)|}{\delta},
$$

where the limit exists by convexity. Then the following inequality holds (see Theorem C.1):

$$
\mu(f \leqslant a-t) \mu^{*}(f \geqslant a,|\nabla f| \leqslant c) \leqslant e^{-\frac{1}{4}(t / c)^{2}} \quad \forall a \in \mathbb{R}, \forall t, c \in(0, \infty),
$$

where $\mu^{*}$ is the outer measure (to avoid the issue of measurability of $|\nabla f|$ ).

Note that, if we fix $a, c$ such that $\mu^{*}(f \geqslant a,|\nabla f| \leqslant c)>0$, relation (5.19) gives a bound on the left tail $\mu(f \leqslant a-t)$ for all $t>0$.

Proof of Proposition 5.5. We can set $z=0$, since the law of $u_{\Lambda}^{\varepsilon}(z)$ in (5.16) does not depend on $z$, and we write $u_{\Lambda}^{\varepsilon}:=u_{\Lambda}^{\varepsilon}(0)$. We denote by $\mathcal{H}_{\varepsilon}^{\xi}(B)$ the argument of the exponential in (5.16), so that $u_{\Lambda}^{\varepsilon}=\mathrm{E}\left[\exp \left(\mathcal{H}_{\varepsilon}^{\xi}(B)\right)\right]$. We also introduce the shorthand

$$
\langle j(B), \xi\rangle:=\iint_{(s, x) \in \Lambda} j\left(B_{s}-x\right) \xi(s, x) \mathrm{d} s \mathrm{~d} x .
$$

We start with a second moment computation:

$$
\mathbb{E}\left[\left(u_{\Lambda}^{\varepsilon}\right)^{2}\right]=\mathrm{E}\left[\mathbb{E}\left[e^{\mathcal{H}_{\varepsilon}^{\xi}(B)+\mathcal{H}_{\varepsilon}^{\xi}(\widetilde{B})}\right]\right]=\mathrm{E}\left[e^{\beta_{\varepsilon}^{2} \mathcal{L}_{\Lambda}(B, \widetilde{B})}\right],
$$

where $B, \widetilde{B}$ are independent Brownian motions, and $\mathcal{L}_{\Lambda}(B, \widetilde{B})$ is their overlap on $\Lambda$ :

$$
\mathcal{L}_{\Lambda}(B, \widetilde{B}):=\iint_{(s, x) \in \Lambda} j\left(B_{s}-x\right) j\left(\widetilde{B}_{s}-x\right) \mathrm{d} s \mathrm{~d} x .
$$

Note that $u_{\Lambda}^{\varepsilon}$ is a function of the white noise $\xi$, so we can define

$$
h_{\varepsilon}(\xi):=\log u_{\Lambda}^{\varepsilon} .
$$


The function $h_{\varepsilon}(\cdot)$ is convex by Hölder's inequality, because $\xi \mapsto\langle j(B), \xi\rangle$ is linear (more precisely, we can ensure that $h_{\varepsilon}(\cdot)$ is convex by choosing a suitable version of the stochastic integral $\langle j(B), \xi\rangle$; see Appendix (D). Then (5.17) follows by (5.19) if we show that $\mu^{*}\left(h_{\varepsilon} \geqslant\right.$ $\left.a,\left|\nabla h_{\varepsilon}\right| \leqslant c\right)$ is uniformly bounded from below, for $a=-\log 2$ and for suitable $c=c_{\hat{\beta}}$.

We need to evaluate the maximal gradient $\left|\nabla h_{\varepsilon}(\xi)\right|$, see (5.18). We define a Gibbs change of measure $\mathbf{P}^{\xi}$ on the Brownian path $B=\left(B_{s}\right)_{s \geqslant 0}$ by

$$
\frac{\mathrm{d} \mathbf{P}^{\xi}}{\mathrm{dP}}(B):=\frac{e^{\mathcal{H}}{ }_{\varepsilon}^{\xi}(B)}{u_{\Lambda}^{\varepsilon}} .
$$

Let us fix $f \in H=L^{2}\left([0, \infty) \times \mathbb{R}^{2}\right)$. Recalling (5.20) and (5.23), we have

$$
\begin{aligned}
\lim _{\delta \downarrow 0} \frac{h_{\varepsilon}(\xi+\delta f)-h_{\varepsilon}(\xi)}{\delta} & =\lim _{\delta \downarrow 0} \frac{1}{\delta} \log \mathbf{E}^{\xi}\left[e^{\beta_{\varepsilon}\langle j(B), \delta f\rangle}\right]=\beta_{\varepsilon} \mathbf{E}^{\xi}[\langle j(B), f\rangle] \\
& =\beta_{\varepsilon} \iint_{(s, x) \in \Lambda} \mathbf{E}^{\xi}\left[j\left(B_{s}-x\right)\right] f(s, x) \mathrm{d} s \mathrm{~d} x .
\end{aligned}
$$

Taking $f$ with $\|f\|_{L^{2}} \leqslant 1$ and recalling (5.22), it follows by Cauchy-Schwarz that

$$
\left|\nabla h_{\varepsilon}(\xi)\right|^{2} \leqslant \beta_{\varepsilon}^{2} \iint_{(s, x) \in \Lambda} \mathbf{E}^{\xi}\left[j\left(B_{s}-y\right)\right]^{2} \mathrm{~d} s \mathrm{~d} y=\frac{\mathrm{E}\left[\beta_{\varepsilon}^{2} \mathcal{L}_{\Lambda}(B, \widetilde{B}) e^{\mathcal{H}_{\varepsilon}^{\xi}(B)+\mathcal{H}_{\varepsilon}^{\xi}(\widetilde{B})}\right]}{\left(u_{\Lambda}^{\varepsilon}\right)^{2}} .
$$

Then, on the event $h_{\varepsilon}(\xi)>a=-\log 2$, i.e. $u_{\Lambda}^{\varepsilon}>\frac{1}{2}$, recalling (5.21), we can bound

$$
\begin{aligned}
\mathbb{E}\left[\left|\nabla h_{\varepsilon}(\xi)\right|^{2} \mathbb{1}_{\left\{h_{\varepsilon}(\xi)>a\right\}}\right] & \leqslant 4 \mathbb{E} \mathrm{E}\left[\beta_{\varepsilon}^{2} \mathcal{L}_{\Lambda}(B, \widetilde{B}) e^{\mathcal{H}_{\varepsilon}(B)+\mathcal{H}_{\varepsilon}(\widetilde{B})}\right] \\
& \leqslant 4 \mathrm{E}\left[\beta_{\varepsilon}^{2} \mathcal{L}_{\Lambda}(B, \widetilde{B}) e^{\beta_{\varepsilon}^{2} \mathcal{L}_{\Lambda}(B, \widetilde{B})}\right] \leqslant \frac{4}{\delta} \mathrm{E}\left[e^{(1+\delta) \beta_{\varepsilon}^{2} \mathcal{L}_{\Lambda}(B, \widetilde{B})}\right],
\end{aligned}
$$

for any $\delta>0$ (by $x \leqslant \frac{1}{\delta} e^{\delta x}$ ). For any subcritical $\hat{\beta} \in(0,1)$, we can fix $\delta=\delta_{\hat{\beta}}>0$ small, so that $\hat{\beta}^{\prime}:=\hat{\beta} \sqrt{1+\delta}<1$ is still subcritical. By (5.21), the last expected value in (5.24) is the second moment of $u_{\Lambda}^{\varepsilon}$ with $\hat{\beta}^{\prime}$ instead of $\hat{\beta}$, hence it is uniformly bounded by some constant $C_{\hat{\beta}}<\infty$, by (5.10) , uniformly over all subsets $\Lambda \subseteq\left(0, \varepsilon^{-2}\right) \times \mathbb{R}^{2}$. Summarizing:

$$
\sup _{\varepsilon>0} \mathbb{E}\left[\left|\nabla h_{\varepsilon}(\xi)\right|^{2} \mathbb{1}_{\left\{h_{\varepsilon}(\xi)>a\right\}}\right] \leqslant C_{\hat{\beta}}^{\prime}<\infty .
$$

We can continue as in the directed polymer case (see Proposition 3.1), noting that

$$
\begin{aligned}
\mu\left(h_{\varepsilon} \geqslant a,\left|\nabla h_{\varepsilon}\right| \leqslant c\right) & =\mu\left(h_{\varepsilon} \geqslant a\right)-\mu\left(h_{\varepsilon} \geqslant a,\left|\nabla h_{\varepsilon}\right|>c\right) \\
& \geqslant \mu\left(h_{\varepsilon} \geqslant a\right)-\frac{1}{c^{2}} \mathbb{E}\left[\left|\nabla h_{\varepsilon}(\xi)\right|^{2} \mathbb{1}_{\left\{h_{\varepsilon}(\xi)>a\right\}}\right] .
\end{aligned}
$$

Since $a:=-\log 2$, we have $\mu\left(h_{\varepsilon} \geqslant a\right)=\mu\left(u_{\Lambda}^{\varepsilon} \geqslant \frac{1}{2}\right) \geqslant\left(4 C_{\hat{\beta}}\right)^{-1}$ as in (3.21). Plugging this bound together with (5.25) into (5.26), we are done by choosing $c=c_{\hat{\beta}}$ large enough.

5.3. Proof of Propositions 5.155.4. Propositions 5.1 and 5.2 are proved repeating almost verbatim the proofs of Propositions 2.1] and 2.2, which are the corresponding results for directed polymers. We omit the details and refer to Subsections 4.1 and 4.2 , 
Proof of Proposition 5.3. We follow closely the proof of Proposition 2.3 in Subsection 4.3, Recall the decomposition $u^{\varepsilon}(z)=u_{A}^{\varepsilon}(z)+\hat{u}_{A}^{\varepsilon}(z)$, see (5.4). Then we further decompose

$$
\hat{u}_{A}^{\varepsilon}(z)=u_{A, C}^{\varepsilon}(z)+u_{A, B}^{\varepsilon}(z)
$$

where $u_{A, C}^{\varepsilon}(z), u_{A, B}^{\varepsilon}(z)$ are defined in analogy with $Z_{N, \beta_{N}}^{A, B}(x), Z_{N, \beta_{N}}^{A, C}(x)$ from (4.9), (4.10):

$$
\begin{aligned}
\left.u_{A, C}^{\varepsilon}(z):=\sum_{k \geqslant 1} \beta_{\varepsilon}^{k} \int_{\substack{\left.0<t_{1}<\cdots<t_{k}<\varepsilon^{-2}, \vec{x} \in\left(\mathbb{R}^{2}\right)^{k} \\
\left\{\left(t_{1}, x_{1}\right), \ldots, t_{k}, x_{k}\right)\right\} \cap C_{\varepsilon}^{z} \neq \varnothing}} \ldots \int_{\left(\mathbb{R}^{2}\right)^{k}} \prod_{i=1}^{k} g_{t_{i}-t_{i-1}}\left(y_{i}-y_{i-1}\right) j\left(y_{i}-x_{i}\right) \mathrm{d} \vec{y}\right) \prod_{i=1}^{k} \xi\left(t_{i}, x_{i}\right) \mathrm{d} t_{i} \mathrm{~d} x_{i}, \\
\left.u_{A, B}^{\varepsilon}(z):=\sum_{k \geqslant 1} \beta_{\varepsilon}^{k} \int_{\substack{\left.\left.0<t_{1}<\cdots<t_{k}<\varepsilon^{-2}, \vec{x} \in\left(\mathbb{R}^{2}\right)^{k} \\
\left\{\left(t_{1}, x_{1}\right), \ldots, t_{k}, x_{k}\right)\right\} \subset A_{\varepsilon}^{z} \cup B_{\varepsilon}^{z} \\
\left\{\left(t_{1}, x_{1}\right), \ldots, t_{k}, x_{k}\right)\right\} \cap B_{\varepsilon}^{z} \neq \varnothing}} \ldots \int_{\left(\mathbb{R}^{2}\right)^{k}} \prod_{i=1}^{k} g_{t_{i}-t_{i-1}}\left(y_{i}-y_{i-1}\right) j\left(y_{i}-x_{i}\right) \mathrm{d} \vec{y}\right) \prod_{i=1}^{k} \xi\left(t_{i}, x_{i}\right) \mathrm{d} t_{i} \mathrm{~d} x_{i}, \\
\end{aligned}
$$

where we set $t_{0}:=0, y_{0}:=\varepsilon^{-1} z$, we recall that $A_{\varepsilon}^{z}$ was defined in (5.3), while $B_{\varepsilon}, C_{\varepsilon}^{z}$ are defined similarly to $B_{N}, C_{N}^{x}$ from (4.7), (4.8) with $N=\varepsilon^{-2}$ and $x=\varepsilon^{-1} z$ : more precisely, recalling $a_{\varepsilon}$ from (5.2), we set

$$
B_{\varepsilon}:=\left(\left(\varepsilon^{-2}\right)^{1-a_{\varepsilon}}, \varepsilon^{-2}\right], \quad C_{\varepsilon}^{z}:=\left\{(t, x) \in \mathbb{R}^{2}: 0<t \leqslant\left(\varepsilon^{-2}\right)^{1-a_{\varepsilon}},\left|x-\varepsilon^{-1} z\right| \geqslant\left(\varepsilon^{-2}\right)^{1-\frac{a_{\varepsilon}}{4}}\right\} .
$$

The proof of Proposition 5.3, similarly to Proposition 2.3, proceeds in three steps.

The first step is to show that $u_{A, C}^{\varepsilon}(x)$ in (5.27) gives a negligible contribution, that is

$$
\sqrt{\log \varepsilon^{-1}} \int_{\mathbb{R}^{2}} \frac{u_{A, C}^{\varepsilon}(z)}{u_{A}^{\varepsilon}(z)} \phi(z) \mathrm{d} z \underset{\varepsilon \rightarrow 0}{\stackrel{L^{2}(\mathbb{P})}{\longrightarrow}} 0 .
$$

The proof is identical to the case for directed polymer, see (4.15) and the following lines. The only difference is that (4.17) will be replaced by its continuum analogue, which is

$$
\mathrm{E}\left[\exp \left\{\left(c_{2 p} \beta_{\varepsilon}\right)^{2} \int_{0}^{\varepsilon^{-2\left(1-a_{\varepsilon}\right)}} J\left(B_{s}^{(1)}-B_{s}^{(2)}\right) \mathrm{d} s\right\} ; \sup _{s \leqslant \varepsilon^{-2\left(1-a_{\varepsilon}\right)}}\left|B^{(i)}(s)\right|>\varepsilon^{-\left(1-\frac{a_{\varepsilon}}{2}\right)}, \text { for } i=1,2\right] \text {, }
$$

where $c_{2 p}:=\sqrt{2 p-1}$ is the hypercontractivity constant for white noise, $B^{(1)}, B^{(2)}$ are two independent Brownian motions and we recall that $J(\cdot)=(j * j)(\cdot)$. The rest of the estimates follow the same lines as in the polymer case.

In view of (5.27) and (5.28), to complete the proof it remains to show that

$$
\sqrt{\frac{\log \varepsilon^{-1}}{2 \pi}}\left\{\int_{\mathbb{R}^{2}} \frac{u_{A, B}^{\varepsilon}(z)}{u_{A}^{\varepsilon}(z)} \phi(z) \mathrm{d} z-\int_{\mathbb{R}^{2}}\left(u_{B}^{\varepsilon} \geqslant(z)-1\right) \phi(z) \mathrm{d} z\right\} \underset{\varepsilon \rightarrow 0}{\stackrel{L^{1}(\mathbb{P})}{\longrightarrow}} 0 .
$$

For $u_{A, B}^{\varepsilon}(z)$ we can give an expression analogous to (4.12), integrating over the last point $(t, w) \in A_{\varepsilon}^{z}$ and the first point $(r, v) \in B_{\varepsilon}$ :

$$
\begin{gathered}
u_{A, B}^{\varepsilon}(z)=\int_{\substack{(t, w) \in A_{\varepsilon}^{z}, w^{\prime} \in \mathbb{R}^{2} \\
(r, v) \in B_{\varepsilon}, v^{\prime} \in \mathbb{R}^{2}}} u_{A}^{\varepsilon}\left(0, z ; \mathrm{d} t, \mathrm{~d} w, \mathrm{~d} w^{\prime}\right) \cdot g_{r-t}\left(v^{\prime}-w^{\prime}\right) j\left(v^{\prime}-v\right) \beta_{\varepsilon} \xi(r, v) \mathrm{d} r \mathrm{~d} v \mathrm{~d} v^{\prime} \\
\cdot u^{\varepsilon}\left(r, v^{\prime} ; \varepsilon^{-2}, \cdot\right),
\end{gathered}
$$

where $u_{A}^{\varepsilon}\left(0, z ; \mathrm{d} t, \mathrm{~d} w, \mathrm{~d} w^{\prime}\right)$ is the "point-to-point" partition function from $(0, z)$ to $\left(t, w, w^{\prime}\right)$, similar to (4.13) (the extra space variable $w^{\prime}$ is due to the convolution with $j(\cdot)$ ), which is 
defined as follows, where we set $t_{0}:=0$ and $y_{0}:=\varepsilon^{-1} z$ :

$$
\begin{gathered}
u_{A}^{\varepsilon}\left(0, z ; \mathrm{d} t, \mathrm{~d} w, \mathrm{~d} w^{\prime}\right):=\sum_{k \geqslant 1} \beta_{\varepsilon}^{k}\left\{\int _ { \substack { 0 < t _ { 1 } < \cdots < t _ { k - 1 } < \varepsilon ^ { - 2 } \\
( x _ { 1 } , \ldots , x _ { k - 1 } ) \in ( \mathbb { R } ^ { 2 } ) ^ { k - 1 } \\
\{ ( t _ { 1 } , x _ { 1 } ) , \ldots , ( t _ { k - 1 } , x _ { k - 1 } ) \} \subset A _ { \varepsilon } ^ { z } } } \left(\int_{\left(\mathbb{R}^{2}\right)^{k-1}} \prod_{i=1}^{k-1} g_{t_{i}-t_{i-1}}\left(y_{i}-y_{i-1}\right) j\left(y_{i}-x_{i}\right)\right.\right. \\
\left.\left.\cdot g_{t-t_{k-1}}\left(w^{\prime}-y_{k-1}\right) \mathrm{d} \vec{y}\right) \prod_{i=1}^{k-1} \xi\left(t_{i}, x_{i}\right) \mathrm{d} t_{i} \mathrm{~d} x_{i}\right\} \\
\cdot j\left(w^{\prime}-w\right) \xi(t, w) \mathrm{d} t \mathrm{~d} w \mathrm{~d} w^{\prime},
\end{gathered}
$$

while $u^{\varepsilon}\left(r, v^{\prime} ; \varepsilon^{-2}, \cdot\right)$ is the "point-to-plane" partition from $\left(r, v^{\prime}\right)$ until time $\varepsilon^{-2}$, defined by (5.9) where we set $t_{0}:=r, y_{0}:=v^{\prime}$ and we replace $0<t_{1}<\cdots$ by $r<t_{1}<\cdots$.

In order to prove (5.29), as in the polymer case, we need two more steps: the second step is to prove that the contribution from $r<\left(\varepsilon^{-2}\right)^{1-9 a_{\varepsilon} / 40}$ to the decomposition (5.30) is negligible; the third step is to show that we can replace $g_{r-t}\left(z^{\prime}-w^{\prime}\right)$ by $g_{r}\left(z^{\prime}-\varepsilon^{-1} z\right)$ in (5.30), because their difference is negligible for (5.29). These steps are proved using exactly the same analysis as in the polymer case, see Subsection 4.3 .

Finally, when we restrict the integral in (5.30) to $r \geqslant\left(\varepsilon^{-2}\right)^{1-9 a_{\varepsilon} / 40}$, i.e. to $(r, z) \in B_{\varepsilon}^{\geqslant}$ (recall (5.6) $)$, and we replace $g_{r-t}\left(z^{\prime}-w^{\prime}\right)$ by $g_{r}\left(z^{\prime}-\varepsilon^{-1} z\right)$, the right hand side of (5.30) becomes exactly $u_{A}^{\varepsilon}(z)\left(u_{B \geqslant}^{\varepsilon}(z)-1\right)$, which proves (5.29).

Proof of Proposition 5.4. In principle, also this last result could be proved as in the polymer case, see Subsection 4.4, using a continuum analogue of Proposition 4.1. However, it is simpler to deduce it from Proposition 2.4 approximating $u_{B \geqslant}^{\varepsilon}(z)$ in $L^{2}(\mathbb{P})$ by a directed polymer partition function $Z_{N, \beta_{N}}^{B \geqslant}(x)$ with $N=\varepsilon^{-2}, x=\varepsilon^{-1} z$ built on the same probability space. The details are described in Section 9 in [CSZ17b] (where the space-time fluctuations of $u^{\varepsilon}(\cdot, \cdot)$ are shown to converge to the solution of the additive SHE).

\section{Appendix A. Scaling Relations fOr KPZ}

We prove a scaling relation between the solutions of the mollified KPZ equations with different parameters. See also [CD18, Section 2]. In particular, we will verify the identity (1.8) which relates the solution of the mollified KPZ equation with the small parameter $\beta_{\varepsilon}$ either in front of the noise or in front of the non-linearity.

Given $\nu, \lambda, D>0$, let $\psi^{\varepsilon}:=\psi^{\varepsilon ; \nu, \lambda, D}$ denote the solution of the mollified KPZ equation

$$
\partial_{t} \psi^{\varepsilon}=\frac{\nu}{2} \Delta \psi^{\varepsilon}+\frac{\lambda}{2}\left|\nabla \psi^{\varepsilon}\right|^{2}+\sqrt{D} \xi^{\varepsilon}, \quad x \in \mathbb{R}^{2}, t \geqslant 0, \text { and } \psi^{\varepsilon}(0, \cdot) \equiv 0,
$$

where $\xi^{\varepsilon}(t, x)$ is the mollification of the space-time white noise $\xi$ in space with $j_{\varepsilon}(x)=$ $\varepsilon^{-2} j(x / \varepsilon)$, and $j \in C_{c}\left(\mathbb{R}^{2}\right)$ is a probability density on $\mathbb{R}^{2}$ with $j(x)=j(-x)$.

Proposition A.1. Let $\psi^{\varepsilon ; \nu, \lambda, D}$ be defined as above. Then for any $a>0$, we have

$$
\left(\psi^{\varepsilon ; \nu, \lambda, D}(t, x)\right)_{t \geqslant 0, x \in \mathbb{R}^{2}} \stackrel{\text { dist }}{=}\left(\frac{\nu}{\lambda} \psi^{a \varepsilon ; 1,1, \beta^{2}}\left(a^{2} \nu t, a x\right)\right)_{t \geqslant 0, x \in \mathbb{R}^{2}},
$$

where $\beta^{2}:=\frac{\lambda^{2} D}{\nu^{3}}$, known as the effective coupling constant, see [CDW10]. 
Remark A.2. In (A.2), setting $a=1, \nu=1, \lambda:=\beta_{\varepsilon}=\hat{\beta} \sqrt{\frac{2 \pi}{\log \varepsilon^{-1}}}$ and $D=1$ gives (1.8), since the constant term $C_{\varepsilon}$ in (1.3) only shifts the solution deterministically.

We need the following scaling relation for the mollified white noise $\xi^{\varepsilon}$.

Lemma A.3. Let $\xi$ be the space-time white noise on $\mathbb{R} \times \mathbb{R}^{2}$ and let $\xi^{\varepsilon}:=\xi * j_{\varepsilon}$, where $j_{\varepsilon}(x)=\varepsilon^{-2} j(x / \varepsilon)$. Then for any $a>0$ and $\nu>0$, we have

$$
\xi^{\varepsilon}\left(\nu a^{2} \cdot, a \cdot\right) \stackrel{\text { dist }}{=} \frac{1}{a^{2} \sqrt{\nu}} \xi^{\frac{\varepsilon}{a}}(\cdot, \cdot)
$$

in the sense that for all $\phi \in C_{c}^{\infty}\left(\mathbb{R} \times \mathbb{R}^{2}\right)$,

$$
\int \phi(t, x) \xi^{\varepsilon}\left(\nu a^{2} t, a x\right) \mathrm{d} t \mathrm{~d} x \stackrel{\text { dist }}{=} \frac{1}{a^{2} \sqrt{\nu}} \int \phi(t, x) \xi^{\frac{\varepsilon}{a}}(t, x) \mathrm{d} t \mathrm{~d} x .
$$

Proof. Since both sides of (A.4) are centered normal random variables, it suffices to check that their variances equal. Note that

$$
\begin{aligned}
X:=\int_{\mathbb{R} \times \mathbb{R}^{2}} \phi(t, x) \xi^{\varepsilon}\left(a^{2} \nu t, a x\right) \mathrm{d} t \mathrm{~d} x & =\int_{\mathbb{R} \times \mathbb{R}^{2} \times \mathbb{R}^{2}} \phi(t, x) \varepsilon^{-2} j\left(\frac{a x-y}{\varepsilon}\right) \xi\left(a^{2} \nu t, y\right) \mathrm{d} t \mathrm{~d} x \mathrm{~d} y \\
& =\frac{1}{a^{4} \nu \varepsilon^{2}} \int_{\mathbb{R}_{\mathbb{R}^{2}}}\left(\int_{\mathbb{R}^{2}} \phi\left(\frac{\widetilde{t}}{a^{2} \nu}, \frac{\widetilde{x}}{a}\right) j\left(\frac{\tilde{x}-\widetilde{y}}{\varepsilon}\right) \mathrm{d} \tilde{x}\right) \xi(\tilde{t}, \widetilde{y}) \mathrm{d} \tilde{\mathrm{d}} \mathrm{y} .
\end{aligned}
$$

Therefore

$$
\begin{aligned}
\operatorname{Var}(X) & =\frac{1}{a^{8} \nu^{2} \varepsilon^{4}} \int_{\mathbb{R} \times \mathbb{R}^{2}}\left(\int_{\mathbb{R}^{2}} \phi\left(\frac{\tilde{t}}{a^{2} \nu}, \frac{\widetilde{x}}{a}\right) j\left(\frac{\tilde{x}-\widetilde{y}}{\varepsilon}\right) \mathrm{d} \tilde{x}\right)^{2} \mathrm{~d} \tilde{t} \mathrm{~d} \widetilde{y} \\
& =\frac{1}{\nu \varepsilon^{4}} \int_{\mathbb{R} \times \mathbb{R}^{2}}\left(\int_{\mathbb{R}^{2}} \phi(t, x) j\left(\frac{a(x-y)}{\varepsilon}\right) \mathrm{d} x\right)^{2} \mathrm{~d} t \mathrm{~d} y .
\end{aligned}
$$

On the other hand,

$$
Y:=\frac{1}{a^{2} \sqrt{\nu}} \int_{\mathbb{R} \times \mathbb{R}^{2}} \phi(t, x) \xi^{\frac{\varepsilon}{a}}(t, x) \mathrm{d} t \mathrm{~d} x=\frac{1}{a^{2} \sqrt{\nu}} \int_{\mathbb{R} \times \mathbb{R}^{2} \times \mathbb{R}^{2}} \phi(t, x) \frac{a^{2}}{\varepsilon^{2}} j\left(\frac{a(x-y)}{\varepsilon}\right) \xi(t, y) \mathrm{d} t \mathrm{~d} x \mathrm{~d} y .
$$

Therefore

$$
\operatorname{Var}(Y)=\frac{1}{\nu \varepsilon^{4}} \int_{\mathbb{R} \times \mathbb{R}^{2}}\left(\int_{\mathbb{R}^{2}} \phi(t, x) j\left(\frac{a(x-y)}{\varepsilon}\right) \mathrm{d} x\right)^{2} \mathrm{~d} t \mathrm{~d} y .
$$

Note that the two variances agree, so we are done.

Proof of Proposition A.1. For $a, b, \widetilde{\varepsilon}, \beta>0$ to be chosen later, define

$$
g(t, x):=b \psi^{\widetilde{\varepsilon} ; 1,1, \beta^{2}}\left(a^{2} \nu t, a x\right) .
$$

By (A.1), we have

$$
\partial_{t} \psi^{\widetilde{\varepsilon} ; 1,1, \beta^{2}}=\frac{1}{2} \Delta \psi^{\widetilde{\varepsilon} ; 1,1, \beta^{2}}+\frac{1}{2}\left|\nabla \psi^{\widetilde{\varepsilon} ; 1,1, \beta^{2}}\right|^{2}+\beta \xi^{\widetilde{\varepsilon}} .
$$


Therefore

$$
\begin{aligned}
\frac{\partial g}{\partial t}(t, x) & =a^{2} \nu b \frac{\partial \psi^{\widetilde{\varepsilon}, 1,1, \beta^{2}}}{\partial t}\left(a^{2} \nu t, a x\right) \\
& =\frac{a^{2} \nu b}{2} \Delta \psi^{\widetilde{\varepsilon}, 1,1, \beta^{2}}\left(a^{2} \nu t, a x\right)+\frac{a^{2} \nu b}{2}\left|\nabla \psi^{\widetilde{\varepsilon}, 1,1, \beta^{2}}\left(a^{2} \nu t, a x\right)\right|^{2}+a^{2} \nu b \beta \xi^{\widetilde{\varepsilon}}\left(a^{2} \nu t, a x\right) \\
& \stackrel{\operatorname{dist}}{=} \frac{\nu}{2} \Delta g(t, x)+\frac{\nu}{2 b}|\nabla g(t, x)|^{2}+b \beta \sqrt{\nu} \xi^{\widetilde{\varepsilon}}(t, x),
\end{aligned}
$$

where we used (A.3).

To find $a, b, \widetilde{\varepsilon}$ and $\beta$ such that $g$ solves (A.1) with parameters $\nu, \lambda, D$, they should satisfy

$$
\varepsilon=\frac{\widetilde{\varepsilon}}{a}, \quad \lambda=\frac{\nu}{b}, \quad D=b^{2} \beta^{2} \nu
$$

There fore we must have

$$
b=\frac{\nu}{\lambda}, \quad \widetilde{\varepsilon}=a \varepsilon, \quad \beta^{2}=\frac{D \lambda^{2}}{\nu^{3}},
$$

while we are free to choose $a>0$. This proves (A.2).

\section{Appendix B. Hypercontractivity of POLYNOMIAL CHAOS}

We recall and refine the hypercontractivity property of polynomial chaos established in [MOO10]. Let $\left(\xi_{i}\right)_{i \in \mathbb{T}}$ be i.i.d. random variables, labeled by a countable set $\mathbb{T}$, with

$$
\mathbb{E}\left[\xi_{i}\right]=0, \quad \mathbb{E}\left[\xi_{i}^{2}\right]=1 .
$$

For every $k \in \mathbb{N}$, let $X_{k}$ be a multi-linear homogeneous polynomial of degree $k$ in the $\xi_{i}$ 's, i.e.

$$
X_{k}=\sum_{I \subseteq \mathbb{T}:|I|=k} f_{k}(I) \prod_{i \in I} \xi_{i},
$$

where $f_{k}(I)$ are real coefficients. For $k=0$, let $X_{0}=f_{0} \in \mathbb{R}$ be a constant. Then for $k \geqslant 1$

$$
\mathbb{E}\left[X_{k}\right]=0, \quad \mathbb{E}\left[X_{k}^{2}\right]=\sum_{I \subseteq \mathbb{T}:|I|=k} f_{k}(I)^{2} .
$$

If we assume that

$$
\sum_{k \in \mathbb{N}} \sum_{I \subseteq \mathbb{T}:|I|=k} f_{k}(I)^{2}<\infty,
$$

then the series $X:=\sum_{k=0}^{\infty} X_{k}$ is easily seen to define an $L^{2}$ random variable. The next key result allows to control higher moments of $X$ in terms of second moments.

It is useful to allow the law of the $\xi_{i}=\xi_{i}^{(N)}$ to depend on a parameter $N \in \mathbb{N}$.

Theorem B.1 (Hypercontractivity). For $N \in \mathbb{N}$, let $\left(\xi_{i}=\xi_{i}^{(N)}\right)_{i \in \mathbb{T}}$ be i.i.d. such that

$$
\mathbb{E}\left[\xi_{i}^{(N)}\right]=0, \quad \mathbb{E}\left[\left(\xi_{i}^{(N)}\right)^{2}\right]=1, \quad \exists p_{0} \in(2, \infty): \quad \sup _{N \in \mathbb{N}} \mathbb{E}\left[\left|\xi_{i}^{(N)}\right|^{p_{0}}\right]<\infty .
$$

Then, for every $p \in\left(2, p_{0}\right)$, there exists a constant $c_{p} \in(1, \infty)$ with the following property: for any choice of coefficients $\left\{f_{k}(I)\right\}_{k \in \mathbb{N}, I \subseteq \mathbb{T},|I|=k}$ satisfying (B.3), if we define $X_{k}$ by (B.1), then the $p$-th moment of the random variable $X=\sum_{k=0}^{\infty} X_{k}$ can be bounded as

$$
\mathbb{E}\left[\left|\sum_{k=0}^{\infty} X_{k}\right|^{p}\right] \leqslant\left(\sum_{k=0}^{\infty}\left(c_{p}^{k}\right)^{2} \mathbb{E}\left[X_{k}^{2}\right]\right)^{p / 2},
$$


with $\mathbb{E}\left[X_{k}^{2}\right]$ given in (B.2). The constant $c_{p}$ only depends on the laws of $\left(\xi_{i}^{(N)}\right)$ and satisfies

$$
\lim _{p \downarrow 2} c_{p}=1 .
$$

Except for relation (B.6), which we prove below, this theorem was proved in MOO10 as an extension of the corresponding result in the Gaussian framework, see [J97. In fact, [MOO10, Proposition 3.16] gave the following explicit bound on $c_{p}$ :

$$
c_{p} \leqslant \widetilde{c}_{p}=2 \sqrt{p-1} \sup _{N \in \mathbb{N}} \frac{\mathbb{E}\left[\left|\xi_{i}^{(N)}\right|^{p}\right]^{1 / p}}{\mathbb{E}\left[\left|\xi_{i}^{(N)}\right|^{2}\right]^{1 / 2}}=2 \sqrt{p-1} \sup _{N \in \mathbb{N}} \mathbb{E}\left[\left|\xi_{i}^{(N)}\right|^{p}\right]^{1 / p},
$$

and note that $\lim _{p \downarrow 2} \widetilde{c}_{p}=2$. This extra factor 2 is the byproduct of a non-optimal symmetrization argument in the proof in [MOO10. We now prove (B.6).

Proof of equation (B.6). By [MOO10, Section 3.2], relation (B.5) holds with constant $c_{p}$ if the law of the random vairable $\xi=\xi_{i}$ in (B.1) is $\left(2, p, 1 / c_{p}\right)$-hypercontractive, that is

$$
\forall a \in \mathbb{R}: \quad\left\|a+\frac{1}{c_{p}} \xi\right\|_{p} \leqslant\|a+\xi\|_{2},
$$

where $\|\cdot\|_{p}:=\mathbb{E}\left[|\cdot|^{p}\right]^{1 / p}$ denotes the $L^{p}$ norm. Since we allow the law of $\xi=\xi^{(N)}$ to depend on $N \in \mathbb{N}$, it follows that we can characterize $c_{p}$ as follows:

$$
c_{p}=\inf \left\{c>1:\left\|a+\frac{1}{c} \xi^{(N)}\right\|_{p} \leqslant\left\|a+\xi^{(N)}\right\|_{2} \quad \forall a \in \mathbb{R}, \forall N \in \mathbb{N}\right\} .
$$

For simplicity, we split the proof in two steps.

Step 1. We first consider the case of a fixed law for the random variable $\xi$ (independent of $N \in \mathbb{N}$ ) satisfying $(\overline{\mathrm{B} .4})$. In view of $(\overline{\mathrm{B} .7})$, we can rephrase our goal $\lim _{p \downarrow 2} c_{p}=1$ as follows:

$$
\forall c>1 \exists p>2: \quad\left\|a+\frac{1}{c} \xi\right\|_{p} \leqslant\|a+\xi\|_{2} \quad \forall a \in \mathbb{R} .
$$

We will first show that given $c>1$, we can find $\bar{p}=\bar{p}_{c, p_{0}}>2$ and $K=K_{c, p_{0}}>0$, such that for all $p \in(2, \bar{p}]$ and $|a|>K$, the inequality in (B.8) holds. We will then find $p \in(2, \bar{p}]$ such that the inequality in (B.8) also holds for all $|a| \leqslant K$.

We first need an elementary estimate: for any $p_{0} \in(2, \infty)$ there exists $C=C_{p_{0}}<\infty$ such that, for all $p \in\left[2, p_{0}\right]$ and $x \in \mathbb{R}$,

$$
|1+x|^{p}=1+p x+\frac{p(p-1)}{2} x^{2}+R(x), \quad \text { with } \quad|R(x)| \leqslant C\left(|x|^{3} \wedge|x|^{p_{0}}\right) .
$$

This follows by Taylor's formula for $|x| \leqslant \frac{1}{2}$ (say) and by the triangle inequality for $|x|>\frac{1}{2}$.

We may assume that $p_{0} \in(2,3]$ in (B.4) (just replace $p_{0}$ by $p_{0} \wedge 3$ ). Then for every $\delta \in \mathbb{R}$ with $|\delta| \leqslant 1$ we can bound

$$
|R(\delta \xi)| \leqslant C\left(|\xi|^{3} \wedge|\xi|^{p_{0}}\right)|\delta|^{p_{0}} \leqslant C\left(1+|\xi|^{p_{0}}\right)|\delta|^{p_{0}} .
$$

Since $\mathbb{E}[\xi]=0$, it follows by (B.4) and (B.9) that for every $\delta \in \mathbb{R}$ with $|\delta| \leqslant 1$

$$
\begin{aligned}
& \mathbb{E}\left[|1+\delta \xi|^{p}\right]=1+\frac{p(p-1)}{2} \delta^{2} \mathbb{E}\left[\xi^{2}\right]+r(\delta) \quad \text { with } \quad|r(\delta)| \leqslant C^{\prime} \delta^{p_{0}}, \\
& \text { where } \quad C^{\prime}=C_{p_{0}}^{\prime}:=C\left(1+\mathbb{E}\left[|\xi|^{p_{0}}\right]\right) .
\end{aligned}
$$

Then, as $|\delta| \rightarrow 0$,

$$
\|1+\delta \xi\|_{p}=1+\frac{p-1}{2} \delta^{2} \mathbb{E}\left[\xi^{2}\right]+O\left(|\delta|^{p_{0}}\right),
$$


uniformly for $p \in\left[2, p_{0}\right]$. This implies that as $|a| \rightarrow \infty$

$$
\begin{aligned}
\frac{\left\|a+\frac{1}{c} \xi\right\|_{p}}{\|a+\xi\|_{2}} & =\frac{\left\|1+\frac{1}{c a} \xi\right\|_{p}}{\left\|1+\frac{1}{a} \xi\right\|_{2}}=\frac{1+\frac{(p-1) \mathbb{E}\left[\xi^{2}\right]}{2 c^{2}|a|^{2}}+O\left(\frac{1}{|a|^{p_{0}}}\right)}{1+\frac{\mathbb{E}\left[\xi^{2}\right]}{2|a|^{2}}+O\left(\frac{1}{|a|^{p_{0}}}\right)} \\
& =1+\left\{\frac{p-1}{c^{2}}-1\right\} \frac{\mathbb{E}\left[\xi^{2}\right]}{2|a|^{2}}+O\left(\frac{1}{|a|^{p_{0}}}\right) .
\end{aligned}
$$

Given $c>1$, we can take $\bar{p}=\bar{p}_{c, p_{0}}:=\min \left\{1+c, p_{0}\right\}>2$ so that uniformly in $p \in(2, \bar{p}]$, the term in bracket is bounded by $c^{-1}-1<0$. Then the RHS of (B.11) is $<1$ for large $|a|$, say for $|a|>K$, where $K=K_{p_{0}, c}<\infty$ only depends on $c$ and $p_{0}$. This proves the inequality in (B.8) for all $p \in(2, \bar{p}]$ and $|a|>K$.

To complete the proof, we now show that there exists $p \in(2, \bar{p}]$ such that the inequality in (B.8) holds for $|a| \leqslant K$. If this is false, then for any sequence $p_{n} \in\left(2, p_{0}\right]$ with $p_{n} \downarrow 2$, we can find $a_{n} \in[-K, K]$ such that

$$
\left\|a_{n}+\frac{1}{c} \xi\right\|_{p_{n}}>\left\|a_{n}+\xi\right\|_{2} \quad \forall n \in \mathbb{N} .
$$

Extracting subsequences, we may assume that $a_{n} \rightarrow a \in[-K, K]$. Since $f(p, a):=\left\|a+\frac{1}{c} \xi\right\|_{p}$ is a continuous function of $(p, a) \in\left[2, p_{0}\right] \times[-K, K]$ (by dominated convergence), we may take the limit of (B.12) as $n \rightarrow \infty$ and get

$$
\left\|a+\frac{1}{c} \xi\right\|_{2} \geqslant\|a+\xi\|_{2},
$$

which is a contradiction, since $\left\|a+\frac{1}{c} \xi\right\|_{2}=\sqrt{a^{2}+\frac{1}{c^{2}} \mathbb{E}\left[\xi^{2}\right]}<\|a+\xi\|_{2}$ (recall that $c>1$ ).

Step 2. Next we allow the law of $\xi=\xi^{(N)}$ to depend on $N \in \mathbb{N}$. In view of (B.7), our goal $\lim _{p \downarrow 2} c_{p}=1$ can be rephrased as follows:

$$
\forall c>1 \exists p>2: \quad\left\|a+\frac{1}{c} \xi^{(N)}\right\|_{p} \leqslant\left\|a+\xi^{(N)}\right\|_{2} \quad \forall a \in \mathbb{R}, \forall N \in \mathbb{N} .
$$

We follow the same proof as in Step 1 . We just need to check the uniformity in $N \in \mathbb{N}$.

Relation (B.10) still holds with $\xi$ replaced by $\xi^{(N)}$, where we stress that $C^{\prime}=C_{p_{0}}^{\prime}<\infty$ because we assume that $\sup _{N \in \mathbb{N}} \mathbb{E}\left[\left|\xi^{(N)}\right|^{p_{0}}\right]<\infty$, see (B.4). Then (B.11) holds as $|a| \rightarrow \infty$, uniformly for $p \in\left[2, p_{0}\right]$ and also for $N \in \mathbb{N}$. This proves that (B.14) holds if we restrict $|a| \leqslant K$, for a suitable $K=K_{p_{0}, c}$ depending only on $c>1$ and $p_{0}$.

It remains to fix $c>1, K<\infty$ and prove that (B.14) holds, for some $p>2$ and for every $|a| \leqslant K$. Arguing again by contradiction, assume now that there are sequences $p_{n} \in\left(2, p_{0}\right]$, $a_{n} \in[-K, K], N_{n} \in \mathbb{N}$, with $p_{n} \downarrow 2$, such that

$$
\left\|a_{n}+\frac{1}{c} \xi^{\left(N_{n}\right)}\right\|_{p_{n}}>\left\|a_{n}+\xi^{\left(N_{n}\right)}\right\|_{2} \quad \forall n \in \mathbb{N} .
$$

Extracting subsequences, we may assume that $a_{n} \rightarrow a \in[-K, K]$, and also that $\xi^{\left(N_{n}\right)}$ converges in law to a random variable $\xi$ (the sequence is tight, by $(\underline{B .4})$ ). Since $\mid a_{N}+$ $\left.\frac{1}{c} \xi^{\left(N_{n}\right)}\right|^{p_{n}}$ are uniformly integrable, again by (B.4), we can take the limit of relation (B.15) and we get precisely $(\mathrm{B} .13)$, which we already showed to be a contradiction.

\section{Appendix C. Gaussian concentration in the Continuum}

We prove a Gaussian concentration result, based on [Led96, Led01, which can be viewed as a "one-sided version" of [FO10, Theorem 2] (cf. (C.6) below with eq. (4) in [FO10]).

Given a probability measure $\mu$ on a measurable space $(E, \mathcal{E})$, we denote by $\mu_{*}$ and $\mu^{*}$ the inner and outer measures: $\mu_{*}(A):=\sup \left\{\mu\left(A^{\prime}\right): A^{\prime} \subseteq B, A^{\prime} \in \mathcal{E}\right\}$ and $\mu^{*}(A)=1-\mu_{*}\left(A^{c}\right)$. 
Theorem C.1. Let $\mu$ be a Gaussian measure on a separable Banach space E, with CameronMartin space $H$ U Let $f: E \rightarrow \mathbb{R} \cup\{-\infty,+\infty\}$ be convex. For $x \in E$ with $|f(x)|<\infty$, define the maximal gradient $|\nabla f(x)| \in[0, \infty]$ in the directions of $H$ by (5.18). Then

$$
\mu(f \leqslant a-t) \mu^{*}(f \geqslant a,|\nabla f| \leqslant c) \leqslant e^{-\frac{1}{4}(t / c)^{2}} \quad \forall a \in \mathbb{R}, \forall t, c \in(0, \infty) .
$$

(The outer measure $\mu^{*}$ appears in (C.1) to avoid the issue of measurability of $|\nabla f|$.)

Let us denote by $\mathcal{K}:=\left\{h \in H:\|h\|_{H} \leqslant 1\right\}$ the unit ball in the Cameron-Martin space $H$. Given a subset $A \subseteq E$, we define its enlargement $A+r \mathcal{K}:=\{x+r h: x \in A, h \in \mathcal{K}\}$. We recall the classical concentration property established by Borell [Led96, Theorem 4.3]:

$$
\begin{gathered}
\forall A \subseteq E \text { with } 0<\mu(A)<1, \quad \text { setting } a:=\Phi^{-1}(\mu(A)), \\
\mu_{*}(A+r \mathcal{K}) \geqslant \Phi(a+r) \quad \forall r \geqslant 0,
\end{gathered}
$$

where $\Phi(x)=\int_{-\infty}^{x} \frac{1}{\sqrt{2 \pi}} e^{-t^{2} / 2} \mathrm{~d} t$ is the standard Gaussian distribution function.

The proof of Theorem C.1 is based on the following Lemma of independent interest, which follows from (C.2). It is close to [Led01, Corollary 1.4] (see also [CTT17, Appendix B.1]).

Lemma C.2. For any measurable subset $A \subseteq E$, the following inequality holds:

$$
\mu(A)\left(1-\mu_{*}(A+r \mathcal{K})\right) \leqslant e^{-\frac{1}{4} r^{2}} \quad \forall r \geqslant 0 .
$$

Proof. We may assume $0<\mu(A)<1$ (otherwise (C.3) holds trivially) and we apply (C.2):

$$
1-\mu_{*}(A+r \mathcal{K}) \leqslant 1-\Phi(r+a) \leqslant e^{-\frac{1}{2}\left((r+a)^{+}\right)^{2}} \quad \forall r \geqslant 0,
$$

where $x^{+}:=\max \{x, 0\}$ and we used the basic bound $1-\Phi(x) \leqslant e^{-x^{2} / 2}$ for $x \geqslant 0$.

Consider first the case $\mu(A) \geqslant \frac{1}{2}$ : then $a=\Phi^{-1}(\mu(A)) \geqslant 0$ and $(r+a)^{+} \geqslant r$, so (C.3) follows by (C.4) (just bound $\mu(A) \leqslant 1$ ). Henceforth we take $\mu(A)<\frac{1}{2}$, so $a<0$. Note that

$$
\mu(A)=\Phi(a)=1-\Phi(|a|) \leqslant e^{-\frac{1}{2}|a|^{2}} .
$$

Fix $r \geqslant 0$. If $|a| \geqslant r$, then (C.3) follows by (C.5) (just bound $1-\mu_{*}(A+r \mathcal{K}) \leqslant 1$ ). If $|a|<r$, then $(r+a)^{+}=(r-|a|)^{+}=r-|a|$ and relations (C.4)-(C.5) yield

$$
\mu(A)\left(1-\mu_{*}(A+r \mathcal{K})\right) \leqslant e^{-\frac{1}{2}\left\{|a|^{2}+(r-|a|)^{2}\right\}} \leqslant \sup _{x \in \mathbb{R}} e^{-\frac{1}{2}\left\{x^{2}+(r-x)^{2}\right\}}=e^{-\frac{1}{4} r^{2}} .
$$

Proof of Theorem C.1. Fix $x, x^{\prime} \in E$ such that $h:=x^{\prime}-x \in H$. The function $g:[0,1] \rightarrow$ $\mathbb{R}$ defined by $g(s):=f\left((1-s) x+s x^{\prime}\right)=f(x+s h)$ is convex (since $f$ is convex), hence

$$
f\left(x^{\prime}\right)-f(x)=g(1)-g(0) \leqslant g^{\prime}(1-):=\lim _{\varepsilon \downarrow 0} \frac{g(1)-g(1-\varepsilon)}{\varepsilon}=\lim _{\varepsilon \downarrow 0} \frac{f\left(x^{\prime}\right)-f\left(x^{\prime}-\varepsilon h\right)}{\varepsilon} .
$$

Recalling (5.18), we have shown that

$$
f\left(x^{\prime}\right)-f(x) \leqslant\left|\nabla f\left(x^{\prime}\right)\right|\left\|x^{\prime}-x\right\|_{H} .
$$

Let us now set

$$
A:=\{f \leqslant a-t\}, \quad B:=\{f \geqslant a,|\nabla f| \leqslant c\} .
$$

\footnotetext{
$\dagger^{\dagger}$ This means that $H$ is a separable Hilbert space, continuously embedded as a dense subset of the separable Banach space $E$, and $\mu$ is a probability on $E$ that can be described as follows: given any complete orthonormal set $\left(h_{n}\right)_{n \in \mathbb{N}}$ in $H$ and given i.i.d. $N(0,1)$ random variables $\left(Z_{n}\right)_{n \in \mathbb{N}}$, the sequence of random elements $X_{N}:=\sum_{n=1}^{N} Z_{n} h_{n}$ converges a.s. in the space $E$, and $\mu$ is the law on $E$ of the limit $X:=$ $\sum_{n \in \mathbb{N}} Z_{n} h_{n}$. The triple $(H, E, \mu)$ is called an abstract Wiener space. We refer to [Led96] for more details.
} 
In view of Lemma C.2 to prove (C.1) it suffices to show that for any $r<\frac{t}{c}$ we have $B \subseteq(A+r \mathcal{K})^{c}$, i.e. $A+r \mathcal{K} \subseteq B^{c}$. So we fix $x \in A, h \in H$ with $\|h\|_{H}<\frac{t}{c}$ and we show that $x^{\prime}:=x+h \in B^{c}$. Either $f\left(x^{\prime}\right)<a$, and then $x^{\prime} \notin B$, or $f\left(x^{\prime}\right) \geqslant a$, and then (by $x \in A$ )

$$
\left|\nabla f\left(x^{\prime}\right)\right| \geqslant \frac{f\left(x^{\prime}\right)-f(x)}{\left\|x^{\prime}-x\right\|_{H}} \geqslant \frac{a-(a-t)}{\|h\|_{H}}>\frac{t}{t / c}=c,
$$

hence again $x^{\prime} \notin B$. This completes the proof.

\section{Appendix D. Stochastic integral as a linear function}

We formulate a linearity result for the stochastic integral with respect to the white noise $\xi=(\xi(z))_{z \in \mathbb{R}^{d}}$ on $\mathbb{R}^{d}$, which is needed in the proof of Proposition 5.5. Recall that the white noise can be realized as a random element of a separable Banach space $E$ of distributions on $\mathbb{R}^{d}$ (e.g. a negative Hölder space), equipped with its Borel $\sigma$-algebra. Denoting by $\mu$ the law of the white noise on $E$, we will use the probability space $(E, \mu)$ as a canonical construction of $\xi$. We also set $H=L^{2}\left(\mathbb{R}^{d}\right)$.

For any $h \in H$, the stochastic integral $\langle h, \xi\rangle:=\int_{\mathbb{R}^{d}} h(z) \xi(z) \mathrm{d} z \sim N\left(0,\|h\|_{H}^{2}\right)$ is a random variable in $L^{2}(E, \mu)$, so it is not canonically defined for any given $\xi \in E$. The following results guarantees the existence of a convenient version of $\langle h, \xi\rangle$.

Theorem D.1. It is possible to define $\langle h, \xi\rangle$ as a jointly measurable of $(h, \xi) \in H \times E$, with the following properties.

- $\langle h, \xi\rangle$ is a version of the stochastic integral $\int_{\mathbb{R}^{d}} h(z) \xi(z) \mathrm{d} z$, for every $h \in H$.

- For any probability measure $\nu$ on $H$, there is a measurable vector space $V_{\nu} \subseteq E$ with

$$
\mu\left(V_{\nu}\right)=1, \quad V_{\nu}+H=V_{\nu},
$$

such that the following property holds:

$$
\forall \xi, \xi^{\prime} \in V_{\nu}: \quad\left\langle h, \alpha \xi+\alpha^{\prime} \xi^{\prime}\right\rangle=\alpha\langle h, \xi\rangle+\alpha^{\prime}\left\langle h, \xi^{\prime}\right\rangle<\infty \quad \text { for } \nu \text {-a.e. } h \in H, \forall \alpha, \alpha^{\prime} \in \mathbb{R} .
$$

Remark D.2. Given any probability $\nu$ on $H$, we can define $f: E \rightarrow \mathbb{R} \cup\{+\infty\}$ by

$$
f(\xi):=\log \int_{H} e^{\langle h, \xi\rangle} \nu(\mathrm{d} h) .
$$

This function is convex when restricted to the vector space $V_{\nu}$ of Theorem D.1, by (D.1) and Hölder's inequality. If we redefine $f(\xi):=+\infty$ for $\xi \notin V_{\nu}$, we obtain a version of $f$ (recall that $\mu\left(V_{\nu}\right)=1$ ) which is convex on the whole space $E$.

This applies, in particular, to the function $h_{\varepsilon}(\xi):=\log u_{\Lambda}^{\varepsilon}$ in the proof of Proposition 5.5, see (5.23), with $u_{\Lambda}^{\varepsilon}=u_{\Lambda, \xi}^{\varepsilon}(0)$ defined in (5.16). In this case $\mathbb{R}^{d}=\mathbb{R}^{1+2}$ and $\nu$ is the law of

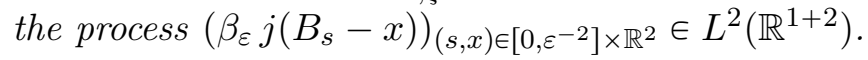

Proof of Theorem D.1. Fix a probability density $\varrho \in C_{c}^{\infty}\left(\mathbb{R}^{d}\right)$ and set $\varrho_{\varepsilon}(z):=\varepsilon^{-d} \varrho\left(\varepsilon^{-1} z\right)$. Also fix a smooth cutoff function $\chi: \mathbb{R}^{d} \rightarrow[0,1]$ with $\chi(x) \equiv 1$ for $|x| \leqslant 1$ and $\chi(x) \equiv 0$ for $|x| \geqslant 2$, and set $\chi_{\varepsilon}(z):=\chi(\varepsilon z)$. For any $h \in H=L^{2}\left(\mathbb{R}^{d}\right)$, we define $h_{\varepsilon} \in C_{c}^{\infty}\left(\mathbb{R}^{d}\right)$ by

$$
h_{\varepsilon}(z):=\chi_{\varepsilon}(z)\left(\varrho_{\varepsilon} * h\right)(z) \text {. }
$$

Since $\lim _{\varepsilon \downarrow 0}\left\|h_{\varepsilon}-h\right\|_{H}=0$, we can find $\left(\varepsilon_{n}=\varepsilon_{n}^{h}\right)_{n \in \mathbb{N}}$ such that $\left\|h_{\varepsilon_{n}}-h\right\|_{H} \leqslant 2^{-n}$. (We can ensure that $\varepsilon_{n}^{h}$ is measurable in $h$, e.g. $\varepsilon_{n}^{h}:=\max \left\{\varepsilon \in\left\{\frac{1}{k}: k \in \mathbb{N}\right\}:\left\|h_{\varepsilon}-h\right\|_{H} \leqslant 2^{-n}\right\}$.) 
For every $n \in \mathbb{N}$ we have $h_{\varepsilon_{n}} \in C_{c}^{\infty}\left(\mathbb{R}^{d}\right)$, hence the map

$$
(h, \xi) \mapsto\langle h, \xi\rangle_{n}:=\left\langle h_{\varepsilon_{n}}, \xi\right\rangle
$$

is canonically defined for any distribution $\xi \in E$, and is jointly measurable in $(h, \xi) \in H \times E$. By the Ito isometry of the stochastic integral and Borel-Cantelli, for any fixed $h \in H$ we have $\lim _{n \rightarrow \infty}\langle h, \xi\rangle_{n}=\langle h, \xi\rangle$ for $\mu$-a.e. $\xi \in E$. We can finally define the measurable map

$$
\langle h, \xi\rangle:=\left\{\begin{array}{ll}
\lim _{n \rightarrow \infty}\langle h, \xi\rangle_{n} & \text { if the limit exists in } \mathbb{R} \\
+\infty & \text { otherwise }
\end{array} .\right.
$$

For every $n \in \mathbb{N}$ the maps $\xi \mapsto\langle h, \xi\rangle_{n}$ are linear, hence the limit map $\xi \mapsto\langle h, \xi\rangle$ is linear too whenever it is finite. More precisely, for every $h \in H$ and $\xi, \zeta \in E$ :

$$
\langle h, \xi\rangle\langle\infty,\langle h, \zeta\rangle<\infty \quad \Longrightarrow \quad\langle h, \alpha \xi+\beta \zeta\rangle=\alpha\langle h, \xi\rangle+\beta\langle h, \zeta\rangle<\infty \quad \forall \alpha, \beta \in \mathbb{R} .
$$

By construction, for every $h \in H$ we have $\langle h, \xi\rangle \in L^{2}(E, \mu)$, so $\langle h, \xi\rangle\langle\infty$ for $\mu$-a.e. $\xi \in E$. If we now fix a probability $\nu$ on $H$, and we define the measurable subset $V_{\nu} \subseteq E$ by

$$
V_{\nu}:=\{\xi \in E:\langle h, \xi\rangle<\infty \text { for } \nu \text {-a.e. } h \in H\},
$$

it follows by Fubini's theorem that $\mu\left(V_{\nu}\right)=1$. Note that $V_{\nu}+H=V_{\nu}$, because $\langle h, g\rangle\langle\infty$ for all $h, g \in H$. Finally, relation (D.4) implies (D.1), which shows that $V_{\nu}$ is a vector space.

\section{ACKNOWLEDGEMENTS}

F.C. is supported by the PRIN Grant 20155PAWZB "Large Scale Random Structures". R.S. is supported by NUS grant R-146-000-253-114. N.Z. is supported by EPRSC through grant EP/R024456/1. We thank MFO, the Oberwolfach Research Institute for Mathematics, for hosting the workshop Scaling Limits in Models of Statistical Mechanics in September 2018, during which part of this work was carried out.

\section{REFERENCES}

[AKQ14] T. Alberts, K. Khanin, J. Quastel. Intermediate Disorder for $1+1$ Dimensional Directed Polymers. Ann. Probab. 42, 1212-1256, 2014.

[BC95] L. Bertini and N. Cancrini. The stochastic heat equation: Feynman-Kac formula and intermittence. J. Stat. Phys. 78, 1377-1401, 1995.

[BC98] L. Bertini and N. Cancrini. The two-dimensional stochastic heat equation: renormalizing a multiplicative noise. J. Phys. A: Math. Gen., 31 (1998), 615-622.

[BDW17] H. Biermé, O. Durieu, Y. Wang. Generalized random fields and Lévy's continuity theorem on the space of tempered distributions. arXiv: 1706.09326v1 (2017).

[CSZ17a] F. Caravenna, R. Sun, N. Zygouras. Polynomial chaos and scaling limits of disordered systems. J. Eur. Math. Soc. 19 (2017), 1-65.

[CSZ17b] F. Caravenna, R. Sun, N. Zygouras. Universality in marginally relevant disordered systems. Ann. Appl. Probab. 27 (2017), 3050-3112.

[CSZ18] F. Caravenna, R. Sun, N. Zygouras. On the moments of the (2+1)-dimensional directed polymer and stochastic heat equation in the critical window. arXiv:1808.03586 (2018).

[CTT17] F. Caravenna, F. L. Toninelli, N. Torri. Universality for the pinning model in the weak coupling regime. Ann. Probab. 45 (2017), 2154-2209.

[CCDW10] L. Canet, H. Chaté, B. Delamotte, N. Wschebor. Nonperturbative renormalization group for the Kardar-Parisi-Zhang equation. Phys. Rev. Lett. 104 (2010), no. 15, 150601.

[CD18] S. Chatterjee, A. Dunlap. Constructing a solution of the $(2+1)$-dimensional KPZ equation. arXiv:1809.00803 (2018).

[C17] F. Comets. Directed polymers in random environments. Lecture Notes in Mathematics 2175 (2017), Springer, Cham. 
[CCM18] F. Comets, C. Cosco, C. Mukherjee. Fluctuation and rate of convergence for the stochastic heat equation in weak disorder. arXiv:1807.03902 (2018).

[CSY04] F. Comets, T. Shiga, N. Yoshida. Probabilistic analysis of directed polymers in a random environment: a review. Stochastic analysis on large scale interacting systems. Adv. Stud. Pure Math. 39 (2004), 115-142.

[C12] I. Corwin. The Kardar-Parisi-Zhang equation and universality class. Random Matrices: Theory and Applications, 1 (2012), 1130001.

[dJ87] P. de Jong. A central limit theorem for generalized quadratic forms. Prob. Th. Rel. Fields 75, 261-277, 1987.

[dJ90] P. de Jong. A central limit theorem for generalized multilinear forms. J. Multivariate Anal. 34, 275-289, 1990.

[DGRZ18] A. Dunlap, Y. Gu, L. Ryzhik, O. Zeitouni. Fluctuations of the solutions to the KPZ equation in dimensions three and higher. arXiv:1812.05768 (2018).

[ET60] P. Erdös, S.J. Taylor. Some problems concerning the structure of random walk paths. Acta Math. Acad. Sci. Hungar. 11 (1960), 137-162.

[EW82] S. Edwards, D. Wilkinson. The surface statistics of a granular aggregate. Proc. R. Soc. Lond. A 381 (1982), 17-31.

[F67] X. Fernique. Processus Linéaires, processus généralisés. Ann. Inst. Fourier 17 (1967), 1-92.

[FO10] P. Friz and H. Oberhauser. A generalized Fernique theorem and applications. Proc. American Math. Soc. 138 (2010), 3679-3688.

[G10] G. Giacomin. Disorder and critical phenomena through basic probability models. École d'Été de Probabilités de Saint-Flour XL - 2010. Springer Lecture Notes in Mathematics 2025.

[G18] Y. Gu. Gaussian fluctuations of the 2D KPZ equation. arXiv:1812.07467 (2018).

[GJ14] P. Gonçalves, M. Jara. Nonlinear fluctuations of weakly asymmetric interacting particle systems. Archive for Rational Mechanics and Analysis 212 (2014), 597-644.

[GIP15] M. Gubinelli, P. Imkeller, N. Perkowski. Paracontrolled distributions and singular PDEs. Forum of Mathematics, Pi (2015) Vol. 3, no. 6.

[GRZ18] Y. Gu, L. Ryzhik, O. Zeitouni. The Edwards-Wilkinson limit of the random heat equation in dimensions three and higher. Comm. Math. Phys. 363 (2018), 351-388.

[H74] A.B. Harris, Effect of random defects on the critical behaviour of Ising models. Journal of Physics C: Solid State Physics, 1974.

[H13] M. Hairer. Solving the KPZ equation. Ann. of Math. 178, 559-664, 2013.

[H14] M. Hairer. A theory of regularity structures. Inventiones Math. 198, 269-504, 2014.

[J97] S. Janson. Gaussian Hilbert spaces. Cambridge Tracts in Mathematics, Vol. 129. Cambridge University Press, Cambridge (1997).

[KPZ86] M. Kardar, G. Parisi, Y.-C. Zhang. Dynamic Scaling of Growing Interfaces. Physical Review Letters 56 (1986), 889-892.

[K16] A. Kupiainen. Renormalization Group and Stochastic PDEs. Ann. Henri Poincaré 17 (2016), 497-535.

[LL10] G.F. Lawler, V. Limic, Random walk: a modern introduction. Cambridge Studies in Advanced Mathematics, 123, Cambridge University Press (2010).

[Led96] M. Ledoux. Isoperimetry and Gaussian analysis. Chapter 3 (pp. 165-294) in Lectures on Probability Theory and Statistics, Ecole d'Eté de Probabilités de Saint-Flour XXIV-1994, Springer (1996).

[Led01] M. Ledoux. The concentration of measure phenomenon. Mathematical Surveys and Monographs, Vol. 89, American Mathematical Society (2001).

[MU18] J. Magnen, J. Unterberger. The scaling limit of the KPZ equation in space dimension 3 and higher. J. Stat. Phys. 171 (2018), Volume 171, 543-598.

[Mor14] G. R. Moreno Flores. On the (strict) positivity of solutions of the stochastic heat equation. Ann. Probab. 42 (2014), 1635-1643.

[MOO10] E. Mossel, R. O'Donnell, K. Oleszkiewicz. Noise stability of functions with low influences: Invariance and optimality. Ann. Math 171 (2010), 295-341.

[MSZ16] C. Mukherjee, A. Shamov, O. Zeitouni. Weak and strong disorder for the stochastic heat equation and continuous directed polymers in $d \geqslant 3$. Electron. Commun. Probab. 21 (2016), paper no. 61 . 
[NPR10] I. Nourdin, G. Peccati, G. Reinert. Invariance principles for homogeneous sums: universality of Gaussian Wiener chaos. Ann. Probab. 38, 1947-1985, 2010.

[NP05] D. Nualart and G. Peccati. Central limit theorems for sequences of multiple stochastic integrals. Ann. Probab. 33, 177-193, 2005.

[QS15] J. Quastel, H. Spohn. The one-dimensional KPZ equation and its universality class. J. Stat. Phys. 160 (2015), 965-984.

[T17] F. L. Toninelli. $(2+1)$-dimensional interface dynamics: mixing time, hydrodynamic limit and Anisotropic KPZ growth. To appear in Proceedings of the International Congress of Mathematicians 2018, arXiv:1711.0557

Dipartimento di Matematica e Applicazioni, Università degli Studi di Milano-Bicocca, via Cozzi 55, 20125 Milano, Italy

E-mail address: francesco.caravenna@unimib.it

Department of Mathematics, National University of Singapore, 10 Lower Kent Ridge RoAD, 119076 Singapore

E-mail address: matsr@nus.edu.sg

Department of Statistics, University of Warwick, Coventry CV4 7AL, UK

E-mail address: N.Zygouras@warwick.ac.uk 